Long-term retention of proprioceptive recalibration

Stefan Maksimovic

Thesis Supervisor

Dr. Erin K. Cressman

Thesis submitted in partial fulfillment of the requirements

for a degree of Master of Science in Human Kinetics

School of Human Kinetics

Faculty of Health Sciences

University of Ottawa

(C) Stefan Maksimovic, Ottawa, Canada, 2017 


\section{LONG-TERM RETENTION OF PROPRIOCEPTIVE RECALIBRATION}

\section{Statement of Contributors}

I hereby declare that I am the sole author of this Master of Science thesis. My contributions included: a review of relevant literature in the area of interest, participant recruitment, participant testing, data collection and analyses, data compilation, statistical analyses, and the write-up of the thesis document. All of these duties were performed under the guidance and mentorship of my research supervisor, Dr. Erin K. Cressman. 


\section{LONG-TERM RETENTION OF PROPRIOCEPTIVE RECALIBRATION}

\section{Acknowledgments}

Two and a half years ago, I never thought I would have chosen the path of a graduate student and learning/performing research, but here I am (or was). This has been a period in my life where I matured, achieved my goals at an academic level and developed professionally.

First and foremost, I'd like to thank Dr. Erin Cressman. She took me on knowing I lacked research experience (leaving me initially puzzled what made her accept me), and made research fun and engaging; two things that I couldn't say I perceived research being before my time in the Sensorimotor Control laboratory. She was an amazing supervisor: incredibly patient, supportive and an excellent motivator. I could not have asked for a better person to learn about life and research from during my Master's. As well, to my committee: Dr. Anthony Carlsen and Dr. Diane Ste-Marie, thank you for providing your time and knowledge throughout the years to me during both my undergraduate and graduate studies; motor control profs really are the best.

To the lab, thank you for all the support, you are the best and I couldn't have asked for a better group of friends. Thank you - Kristin, Darrin, Neil (from Fin), J-M, Christin, Mo \& Kia, Mohamadou, Sajida, Dami and Vicki. Additionally, I need to PARTICULARLY thank all my motivated participants for providing their patience and time, which they without hesitation let me have, so they could participate in my experiment. I couldn't have done it without you.

Finally, I want to thank all the people closest to me in my life; you know who you are! You kept me motivated throughout my Masters, especially in the month of January (when I tested every single day that month). Thank you to everyone for all of your support!! 


\section{Table of Contents}

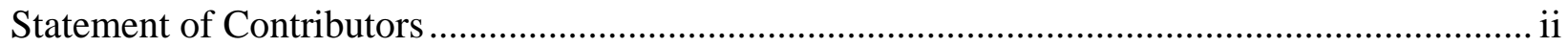

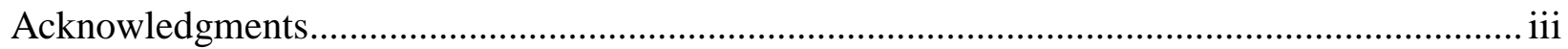

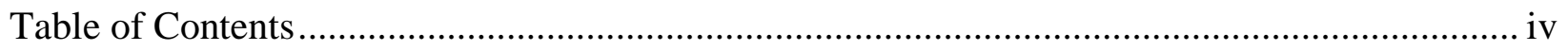

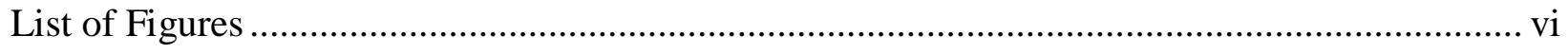

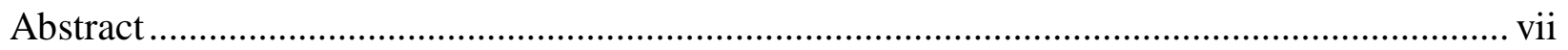

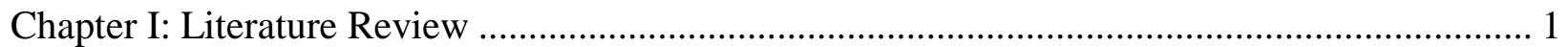

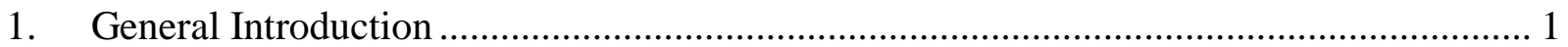

2. Goal-Directed Movements ................................................................................. 3

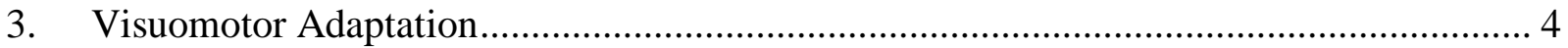

3.1 Time Course of Visuomotor Adaptation .................................................................. 5

3.2 Processes Underlying Visuomotor Adaptation......................................................... 6

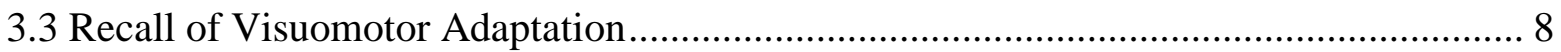

3.4 Savings of Visuomotor Adaptation ............................................................................. 9

4. Processes Underlying Motor Recall and Savings ................................................ 11

5. Proprioceptive Recalibration ............................................................................. 12

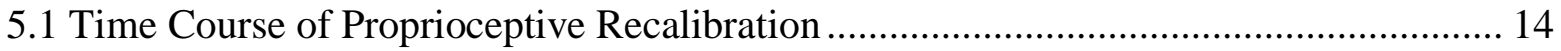

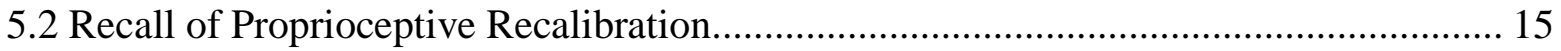

6. Proposed Research and Hypotheses........................................................................ 17

Chapter II: Research Article ......................................................................................... 19

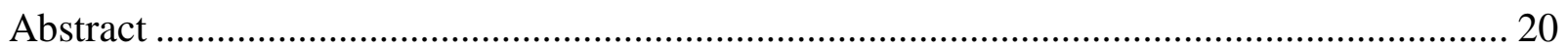

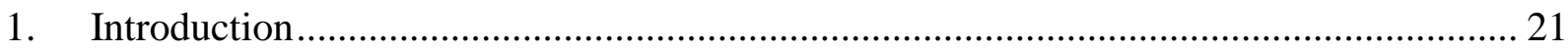

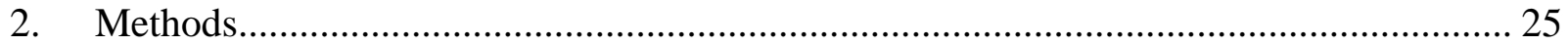

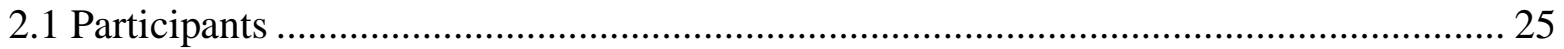

2.2 Experimental Apparatus and Task................................................................... 26

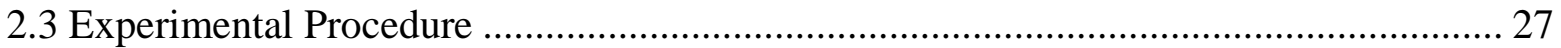

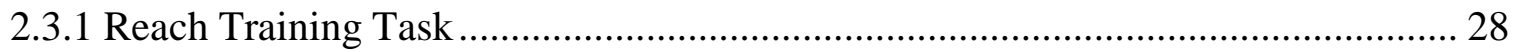

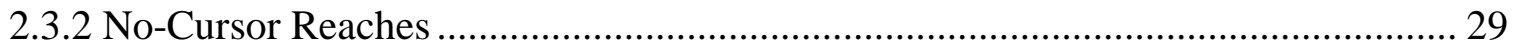

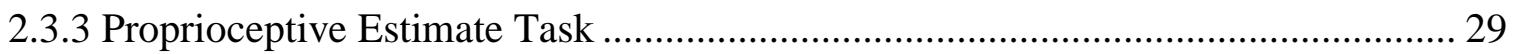

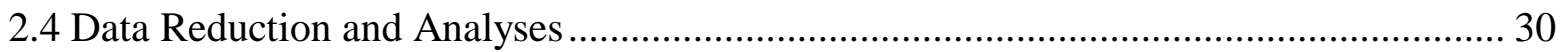

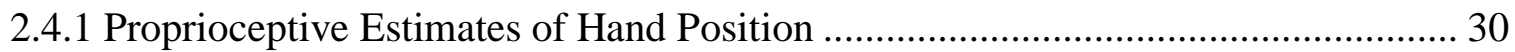

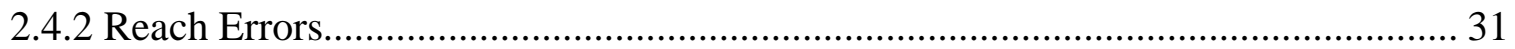

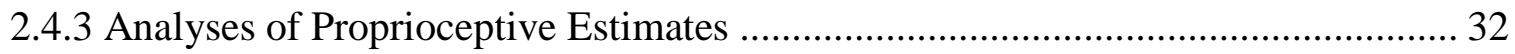




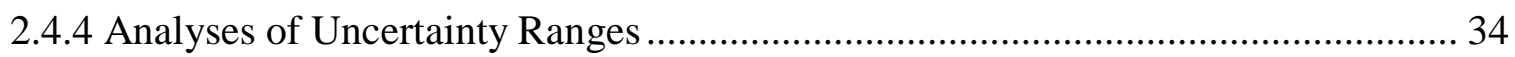

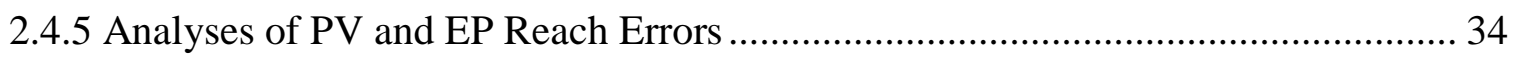

2.4.6 Analyses of Reach Training ........................................................................ 36

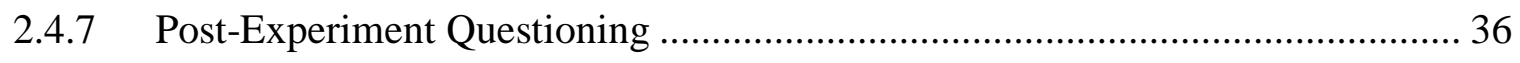

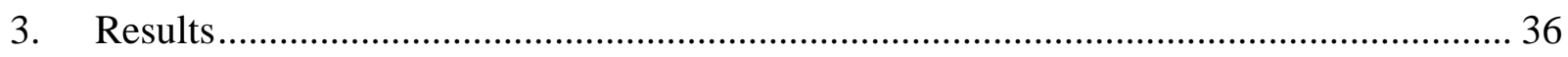

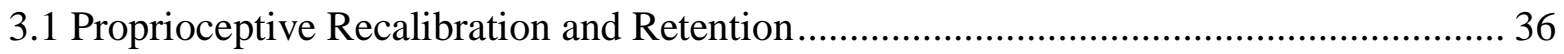

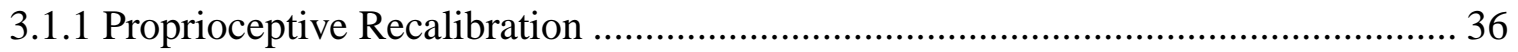

3.1.2 Retention of Proprioceptive Recalibration (Recall) ............................................. 38

3.1.3 Retention of Proprioceptive Recalibration (Savings) ............................................... 39

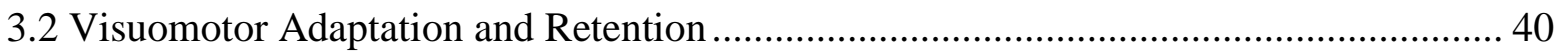

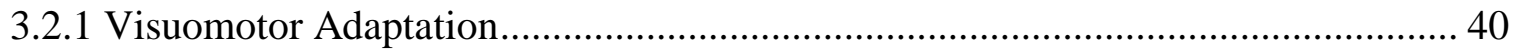

3.2.2 Retention of Visuomotor Adaptation (Recall of Aftereffects) ................................ 41

3.2.3 Retention of Visuomotor Adaptation (Savings) .................................................... 42

3.2.4 Performance during Reach Training Trials............................................................. 44

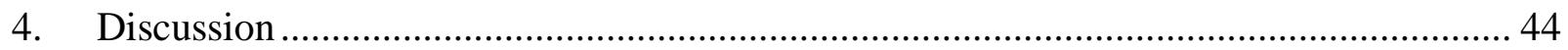

4.1 Proprioceptive Recalibration and Retention................................................................ 45

4.2 Visuomotor Adaptation and Retention .................................................................. 48

4.3 Mechanisms underlying Visuomotor Adaptation and Proprioceptive Recalibration ...... 50

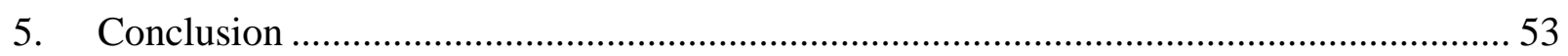

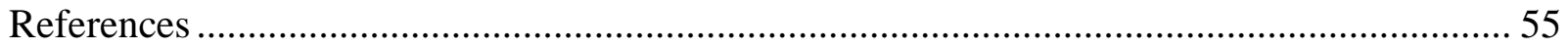

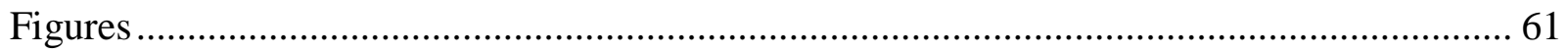

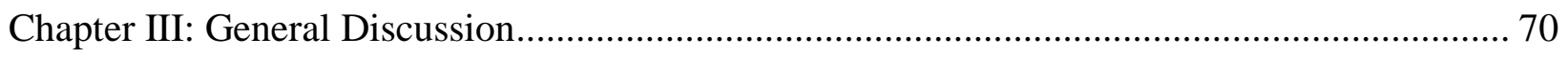

Retention of Proprioceptive Recalibration vs. Retention Visuomotor Adaptation ................... 71

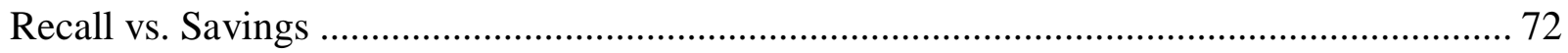

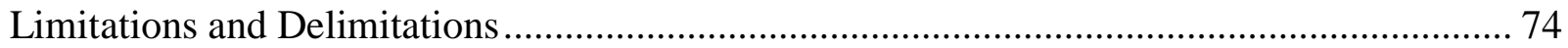

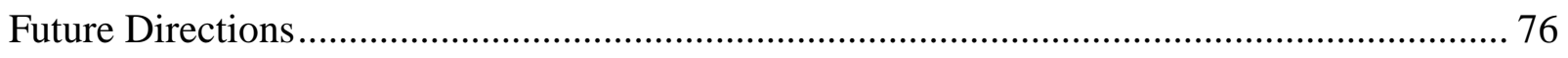

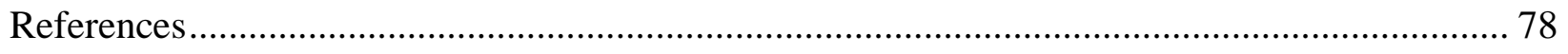

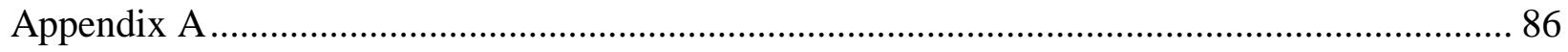

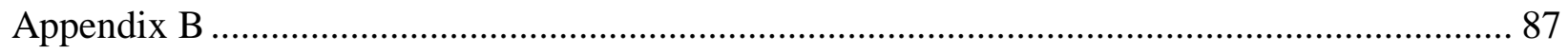

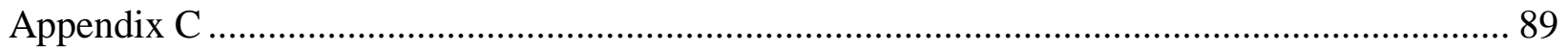




\section{List of Figures}

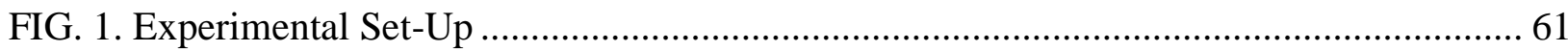

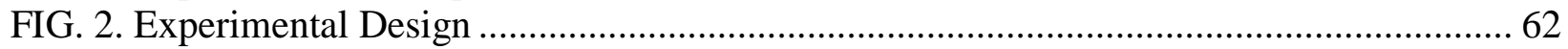

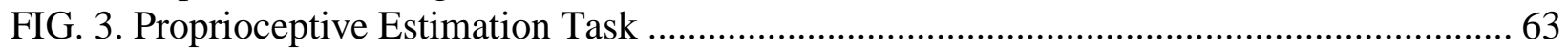

FIG. 4. Mean Proprioceptive Biases and Reach Endpoint Errors.......................................... 64

FIG. 5. Recall of Proprioceptive Recalibration and Visuomotor Adaptation............................ 65

FIG. 6. Mean Proprioceptive Biases and Reach Endpoint Errors across Testing Days .............. 66

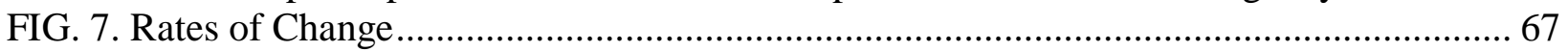

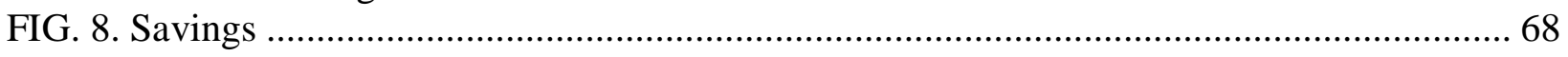

FIG. 9. Reach Performance during Reach Training Trials ................................................. 69 


\title{
LONG-TERM RETENTION OF PROPRIOCEPTIVE RECALIBRATION
}

\begin{abstract}
Proprioception is recalibrated following reaches with misaligned visual feedback of the hand, such that one's sense of felt hand position is shifted in the direction of the visual feedback provided (Cressman \& Henriques 2009). In the current experiment, we examined the ability of proprioceptive recalibration to be retained over an extended period of time (i.e. 4 days), and the benefits of additional training on retention in the form of recall and savings (i.e. faster relearning on subsequent testing days). Twenty-four participants trained to reach to a target while seeing a cursor that was rotated $30^{\circ}$ clockwise relative to their hand on an initial day of testing. Half of the participants then completed additional reach training trials on 4 subsequent testing days (Training group), whereas the second half of participants did not complete additional training (Non-Training group). Participants provided estimates of their felt hand position on all 5 testing days to establish retention of proprioceptive recalibration. Results revealed that proprioceptive recalibration was recalled 24 hours after initial training and that there was no benefit of additional training. Retention in the form of savings was observed on all days for the Training group and on Day 5 in the Non-Training group. These results reveal that proprioceptive recalibration does not benefit from additional training but is retained in the form of recall and savings. Taken together, results from the two groups of participants showed that the sensory system's ability to change over time appeared to saturate early on, within two days of training. Moreover, the different time scales (i.e. 1 day for recall versus 4 days for savings), suggested that distinct processes may underlie recall and savings of proprioceptive recalibration.
\end{abstract}




\section{LONG-TERM RETENTION OF PROPRIOCEPTIVE RECALIBRATION}

\section{Chapter I: Literature Review}

\section{General Introduction}

Humans interact with a constantly changing environment Thus, we need to learn new movement skills and adapt learned ones in order to account for changing conditions. Motor learning encompasses both skill acquisition and motor adaptation and is defined as the process of improving one's performance, leading to enduring changes in the motor system, when encountering novel task demands or novel environments (Wolpert et al. 2001). One approach to studying motor adaptation in the laboratory is to have individuals reach to targets while providing altered visual feedback of their hand by way of a misaligned cursor onscreen and determining how they adapt their movements (Cressman \& Henriques, 2009; Henriques \& Cressman, 2012; Krakauer et al. 2000; Zbib et al. 2016). Reaching with altered visual feedback has been shown to lead to rapid adaptation of one's reaches so that reaching errors are reduced and the cursor is brought back to the target (Cressman \& Henriques, 2009; Krakauer et al. 2000, 2009; Zbib et al. 2016). After adapting their movements, individuals continue to reach with the newly acquired movement pattern, even after the feedback is removed. These reach errors they exhibit, known as aftereffects, are evidence that persistent changes have occurred in the motor system (i.e. motor adaptation) (Baraduc \& Wolpert 2000; Buch et al. 2003; Cressman \& Henriques 2009, 2010; Cressman et al. 2010; Krakauer et al. 1999, 2000; Martin et al. 1996; Zbib et al. 2016). These changes in the motor system are robust in that they have been shown to be recalled up to 12 months following initial training (Kitazawa and Yin 2001; Nourouzpour et al. 2015; Yamamoto et al. 2006). In addition, evidence of retention of visuomotor adaptation can be seen in faster relearning (i.e. savings) when participants are exposed to the same novel 


\section{LONG-TERM RETENTION OF PROPRIOCEPTIVE RECALIBRATION}

environment after a prolonged time interval (one week to month(s)) (Krakauer et al. 2005;

Krakauer, 2009).

In addition to changes in the motor system, the sensory system has been shown to adapt following reaches with misaligned visual feedback of the hand. Specifically, participants recalibrate their sense of felt hand position (i.e. proprioceptive recalibration), such that they feel their hand is shifted in the direction of the visual feedback provided (Cressman \& Henriques 2009; Zbib et al. 2016). There has been limited work looking at the long term retention of proprioceptive recalibration. In fact, changes in the sensory system have only been tested 24 hours after initial training, where evidence of retention was observed (Nourouzpour et al. 2015; Ostry et al. 2010). As well, there has been no previous work looking at retention of proprioceptive recalibration by assessing savings. The present experiment looks to determine the ability proprioceptive recalibration to be retained over a long period of time (i.e. 4 days) by measuring retention before (i.e. recall) and while the cursor perturbation is re-introduced (i.e. savings). As well, we ask if additional training benefits short-term retention of proprioceptive recalibration (i.e. 24 hours).

To address these questions, we first established the time course of proprioceptive recalibration on an initial day of testing. We then looked at the ability of proprioceptive recalibration to be retained across four consecutive days, by testing immediate recall and examining savings when the perturbation was reintroduced. The methods were similar to the experiment by Zbib et al. (2016); however, the timeframe of testing was extended to 5 consecutive days. Research in this area is invaluable to improving the development of rehabilitation programs for individuals with sensorimotor dysfunctions, as the results of this 


\section{LONG-TERM RETENTION OF PROPRIOCEPTIVE RECALIBRATION}

experiment will provide understanding of the potential of rehabilitation techniques to improve or restore sensorimotor function through sensory recalibration during long-term programs.

This literature review will provide the background necessary to understand the specific objectives of the current experiment. To begin, we establish the time course of visuomotor adaptation observed during reach training with distorted visual feedback of the hand. The processes underlying visuomotor adaptation are then explained. Afterwards, retention in the motor system, including how it is measured and related findings are outlined. Finally, we discuss the concept of savings based on findings in current literature. Following our discussion of visuomotor adaptation, we turn our attention to proprioceptive recalibration and the processes that underlie the observed changes. Specifically, we outline the time course of proprioceptive recalibration and retention in the form of recall. The literature review concludes with a discussion of the robustness of sensory changes.

\section{Goal-Directed Movements}

Goal directed movements allow us to interact with various objects, within countless differing environments (Wolpert \& Kawato, 1998). In order to execute goal-directed reaches, a series of sensory to motor transformations need to occur. These transformations are proposed to occur within an internal model framework. The internal model includes two components: an inverse model, which determines the motor commands necessary to produce the desired limb movement, and a forward model, which predicts the sensory consequences of that movement given the current position of the limb and the motor command (Wolpert et al. 2001; Wolpert \& Kawato 1998). Errors predicted by the forward model (i.e. differences between the limb's predicted and actual or desired position) can be utilized by the inverse model to update a motor command (Jeannerod, 2003; Wolpert et al, 1995a). 


\section{LONG-TERM RETENTION OF PROPRIOCEPTIVE RECALIBRATION}

\section{Visuomotor Adaptation}

If an individual reaches and their hand does not achieve the desired target, for example if the provided visual feedback of their actual hand position is altered, the motor plan is no longer suitable. Thus, the internal model representing the movement needs to change to adapt to the altered visual feedback. The reduction of systemic reach errors in order to return to a previous (or baseline) level of performance is known as visuomotor adaptation; a common form of motor learning (Krakauer et al. 2009). Visuomotor adaptation has previously been examined in the laboratory by having participants reach to targets when sensory feedback is manipulated. For example, visual feedback of one's hand position can be manipulated by having participants wear laterally displacing prism goggles or reach in a virtual reality setting where a cursor misrepresents their actual hand position on a screen (Cressman \& Henriques, 2009; Harris, 1963, 1965; Krakauer, 2009; Redding \& Wallace, 1993, 1997b, 2002, 2003; Sainburg \& Wang, 2002). Also, adaptation of movement dynamics has been examined by having participants reach within a velocity-dependent force-field, where velocity dependent loads are applied to the reaching limb and push the hand off-course (Mattar et al. 2011, 2013; Ostry et al. 2010; Shadmehr \& MussaIvaldi, 1994). In order to examine changes in the motor system, these paradigms typically include three testing stages: (1) a baseline stage (or pre-test), in which participants reach in the absence of a perturbation and visual feedback, (2) an adaptation stage, in which participants reach while the perturbation is present and (3) a post-test stage, which is similar to the baseline stage. In general, results indicate that participants tend to produce reaching errors in the post-test stage that are similar to the adapted movements acquired in the adaptation stage, such that they continue to reach as if they were still influenced by the perturbation (Cressman \& Henriques, 2009; Krakauer, 2009; Sainburg \& Wang, 2002; Wolpert et al. 1995b). These changes in reaches 


\section{LONG-TERM RETENTION OF PROPRIOCEPTIVE RECALIBRATION}

in the post-test relative to the pre-test are known as aftereffects and indicate that participants have adapted their movements, meaning that a persistent change has occurred in the motor system (i.e. visuomotor adaptation).

\subsection{Time Course of Visuomotor Adaptation}

Changes in reaches in response to altered visual feedback have been shown to arise quickly. For example, reaches were shown to return to baseline levels of performance (i.e. participants reached so that their hand correctly acquired the target) after completing just 15 reach training trials while wearing laterally displacing prisms (Redding \& Wallace 1993, 2000). Similarly, performance has been shown to return to near-baseline levels when reaching with a cursor that is rotated from one's actual hand position in a virtual reality environment after approximately 20 trials; specifically, when reaching to a single target with a $30^{\circ}$ cursor rotation (Krakauer et al. 2000). Yamamoto et al. (2006) have also shown quick changes in reaches with distorted visual feedback of the hand, such that performance returned to near-baseline levels after 30 trials of training with a $40^{\circ}$ cursor rotation. Finally, visuomotor adaptation has been observed within 20 trials of reaching in a velocity-dependent force (Mattar et al. 2011, 2013; Ostry et al. 2010). Notably, these experiments assessed performance changes during training trials where the perturbation was still present. These observed changes in reaches may not necessarily be the best indication of visuomotor adaptation (Pisella et al. 2004), as participants could potentially use strategic processes that lead to rapid reductions in error, instead of using adapted motor commands indicative of long-term motor changes (Pisella et al. 2004; Redding et al. 2005; Taylor \& Ivry 2011; Taylor et al. 2014; Weiner et al. 1983).

A few experiments have examined visuomotor adaptation by using a post-test with nocursor reaching trials as the primary assessment tool instead of performance changes during 


\section{LONG-TERM RETENTION OF PROPRIOCEPTIVE RECALIBRATION}

training (e.g. Baraduc \& Wolpert 2000; Buch et al. 2003; Cressman \& Henriques 2009, 2010; Cressman et al. 2010; Krakauer et al. 1999, 2000; Martin et al. 1996; Zbib et al. 2016). For example, Cressman \& Henriques (2009) found that immediately after training with a $30^{\circ}$ clockwise $(\mathrm{CW})$ rotation, participants produced aftereffects of about $18^{\circ}$ counter-clockwise (CCW, in the direction opposite the distortion), or about $60-70 \%$ of the distortion initially introduced, indicating that their reaches had been adapted. Furthermore, in an experiment by Zbib et al. (2016), motor changes arose quickly with a significant change in no-cursor reaching trials occurring after only 5 reach training trials. These quickly arising aftereffects do not appear to benefit from additional reach training. Indeed, Salomonczyk et al. (2011) showed that prolonged reach training with a $30^{\circ} \mathrm{CW}$ rotation did not provide additional benefits. In fact, participants maintained an adaptation level of $61 \%$ of the visuomotor distortion across 3 testing blocks of 99 trials, indicating motor changes saturated quickly in the first block of training, Moreover, extending the training trials across days does not appear to benefit aftereffects. Specifically, Wong and Henriques (2009) showed that additional training across 5 days did not benefit retention as there were no differences between aftereffect trials across the 5 days. Taken together, the results in these experiments indicate that aftereffects arise and saturate quickly, demonstrating (short-term) persistent changes in the motor system that do not benefit from additional training.

\subsection{Processes Underlying Visuomotor Adaptation}

In order to explain the rapid changes observed in the motor system during reach training and the persistent changes (i.e. aftereffects) seen following training, it has been suggested that visuomotor adaptation arises as a result of two processes. Early on, work by Redding and colleagues (Redding \& Wallace 1996, 2002, 2003; Redding et al. 2005) suggested adaptation 


\section{LONG-TERM RETENTION OF PROPRIOCEPTIVE RECALIBRATION}

observed in prism literature arose first due to strategic control and then spatial realignment.

Strategic control is the quick improvement of performance through reduction of movement errors and spatial realignment refers to slower adjustments made as a result of misalignments between previous and new sensory states of limb position (without vs. with prism goggles), leading to remapping of the sensorimotor system. It is spatial realignment that is proposed to give rise to aftereffects following prism training. Recently, Taylor and Ivry (2011) have also proposed that visuomotor adaptation arises due to two processes in response to reaching with altered visual feedback of one's hand in a virtual reality environment: an explicit and implicit process. Explicit learning, driven by target error, is due to initially large followed by smaller explorations of aiming direction towards the correct solution, whereas implicit learning, driven by sensoryprediction error, is slow and monotonic. Specifically, participants (explicitly) correct their reaching movements initially in reach training trials so that the cursor achieves the target, while implicit processes are involved in error-based updating of the internal forward model (McDougle et al. 2016; Taylor \& Ivry 2011; Taylor et al. 2014). A similar two-stage model has also been put forward based on results achieved using a force-field adaptation paradigm. For example, Smith et al. (2006) proposed that adaptation is driven by a fast process that has a poor retention rate and leads to the rapid reduction of errors to quickly improves one's performance, and a slow process that retains information well and responds slower to errors throughout training trials. For that reason, the slow process is suggested to be linked with long-term motor changes (Smith et al. 2006). While these three models use different terminology to describe the processes underlying motor adaptation, the models are similar in that there is (1) an initial fast process, potentially involving an explicit mechanism, that leads to quick decreases in error when the perturbation is present and (2) a slower process, potentially involving an implicit mechanism, which leads to 


\section{LONG-TERM RETENTION OF PROPRIOCEPTIVE RECALIBRATION}

adaptation in the motor system as observed through aftereffects (Redding \& Wallace 1996, 2002, 2003; Redding et al. 2005; Smith et al. 2006; Taylor \& Ivry 2011; Taylor et al. 2014).

\subsection{Recall of Visuomotor Adaptation}

Having established that motor changes can be observed within and immediately following reach training, we will now discuss the ability of the motor system to use this this information over longer periods of time. Typically, retention is determined by assessing performance in a post-test stage during a second testing session, completed a couple of hours to days or weeks after the initial training session. Retention of visuomotor adaptation has been observed (24 and 48 hours) following prism adaptation (Yin \& Kitazawa 2001) and at varying times (24 hours, 1-2 week(s) and 12 months) after training with a visuomotor rotation (Nourouzpour et al. 2015; Yamamoto et al. 2006). In the experiment by Yin and Kitazawa (2001), they observed that a monkey was able to adapt his or her reaches while wearing laterally-displacing prism goggles such that aftereffects greater than $50 \%$ of the prism displacement were seen up to 72 hours after completing 500 training trials; these aftereffects were also noticed immediately after completing only 50 training trials. Retention of visuomotor adaptation has also been observed following reach training with a $40^{\circ} \mathrm{CW}$ visuomotor rotation, such that Nourouzpour et al. (2015) found that immediate aftereffects were not different from those measured 24 hours later. Moreover, an experiment by Yamamoto et al. (2006), looked to determine the ability of the motor system to recall information over a longer period of time (1-2 week(s) \& >1 year). They had both rhesus monkeys and human participants learn to use a joystick to move a cursor that was gradually rotated $40^{\circ} \mathrm{CCW}$ relative to the hand across trials. In their experiment, aftereffects were measured immediately following training and then re-tested again on a later date. Yamamoto et al. (2006) showed that participants (monkey and human) continued to show aftereffects after the 


\section{LONG-TERM RETENTION OF PROPRIOCEPTIVE RECALIBRATION}

initial training day. In fact, the monkey recalled $81.6 \%$ of its adapted movements, while humans recalled $81.2 \%$ of their adapted movements following a gap of one week between post-tests and retention tests. In humans specifically, similar levels of recall were also found after a lengthy gap in performance (384-418 days). These results show significant long-lasting changes in motor performance and suggest that even relatively short-term reach training with distorted visual feedback (e.g. 120 trials for humans \& 150 trials for monkeys) leads to long-term changes in the motor system.

\subsection{Savings of Visuomotor Adaptation}

The discussion so far has focused on experiments examining motor retention by way of nocursor reaching trials (i.e. aftereffects trials). Notably, the majority of previous experiments examining motor retention have compared participants' performances during training trials across days while the perturbation was still present. In these experiments, researchers examined the rate at which participants re-learned the task. Thus, the focus was on retention in the form of savings, which is defined as faster relearning when training in a previously experienced environment compared to performance during initial training (Krakauer et al. 2005; Krakauer, 2009).

Savings has been examined in paradigms which include a washout task (Krakauer et al. 2005). In the experiments by Krakauer and colleagues, the paradigm is divided into 3 stages. First, a participant is exposed to a perturbation (e.g. a cursor rotation), to which they adapt. Afterwards, the adapted movements are washed out by having participants perform trials with a counter-rotation. The counter-rotation may be imposed immediately following initial reach training or following a break ranging from 2 to 24 hours. Thus, participants unlearn their adapted reach responses and the stimulus they adapted to no longer evokes the learned response (i.e. 


\section{LONG-TERM RETENTION OF PROPRIOCEPTIVE RECALIBRATION}

there is no more evidence of aftereffects; Krakauer et al. 2005; Smith et al. 2006). The third stage of the paradigm involves re-introducing participants to the perturbation initially introduced. If readaptation occurs more quickly in this third stage compared to the initial stage, then savings are present. In contrast to the savings experiments mentioned in the previous paradigm, this paradigm has the advantage of not requiring an extended break between testing sessions. Results revealed that a small amount of savings occurred after washout was introduced only 5 minutes after the original rotation and at 24 hours; however, it was noted that the washout blocks reduced the maximal amount of savings that were observed as compared to when there was no washout.

Other studies examining savings have given participants a break between training sessions. For example, Klassen et al. (2005) looked at savings 24 hours after participants trained to reach with a cursor that was gradually or abruptly rotated $30^{\circ} \mathrm{CCW}$ relative to the hand. Participants showed similar learning curves on Day 2, regardless of how the distortion was introduced, such that there was an evident increase in the rate of re-learning on Day 2 compared to Day 1. Moreover, in an experiment by Caithness et al. (2004), participants demonstrated savings after performing a reaching task with a $30^{\circ} \mathrm{CCW}$ visuomotor rotation, where participants performed the same reaching task 48 hours later during a second testing session. Specifically, initial reaching errors were approximately $5.5^{\circ}$ in the second session compared to $23.2^{\circ}$ in the first session, suggesting faster re-learning upon re-exposure to the rotation (Caithness et al. 2004). Krakauer et al. (2005) extended the time between testing sessions to 1 week. Interestingly, they found that while savings were evident from groups with break periods of 24 and 48 hours between testing sessions, the greatest savings were observed for participants in which the testing sessions were separated by a 1 week break period. Together, these experiments demonstrate that retention can be evident in the form of savings with an extended break up to 1 week. 


\section{LONG-TERM RETENTION OF PROPRIOCEPTIVE RECALIBRATION}

\section{Processes Underlying Motor Recall and Savings}

Two types of models have been used to explain savings in motor adaptation: a linear timevariant (LTI) (two- or multi-state) model and a non-linear model. Specifically, Smith et al. (2006) has extended their linear model outlined on Page 9 in the Time Course of Visuomotor Adaptation Section, to suggest that savings arises due to the interaction of the fast and slow processes (Smith et al. 2006). According to Smith, re-learning occurs more rapidly than initial learning due to the fact that when participants are reintroduced to the perturbation, the slow process retains information and can contribute to initial changes in reaches (Kojima et al. 2004; Smith et al. 2006). This model has been further extended by Lee and Schweighofer (2009), to include a single fast process and multiple slow processes organized in parallel. Each process is proposed to have its own learning and forgetting rates and states (i.e. the limbs and muscles controlled by the CNS and their positions and velocities) receive contextual cues, which have two roles: to select the appropriate state(s) to be calculated for the total output and to allow the selected state(s) to be updated based on the motor errors. Recently, Berniker and Kording (2011) have suggested that the sources of potential error (be they arising from the body or the environment) and their relevance are considered during initial adaptation and savings. Together, these models suggest that of the two processes (fast and slow processes), the slow process retains information better during reach training and thus drives savings, even in the long-term.

Recently, it has also been proposed that the formation of long-term motor memories in the form of recall and savings are driven by two distinct neural networks. Most previous adaptation experiments have examined the formation of motor memories by using a reaching paradigm (Krakaeur et al. 2005; Smith et al. 2006), however these motor memories have also been examined by way of locomotor adaptation (Malone et al. 2011; Mawase et al. 2014, 2016, 2017). 


\section{LONG-TERM RETENTION OF PROPRIOCEPTIVE RECALIBRATION}

Initially, an experiment by Malone et al. (2011), suggested the possibility that cerebellar activity could be important for strengthening motor re-learning during locomotor adaptation. This was further examined in a locomotion adaptation experiment by Mawase et al. (2017), which examined the neural correlates of locomotor adaptation and retention (in the form of recall and savings) over two days by having participants complete a resting state fMRI after baseline walking on a tied treadmill on Day 1 (i.e. belts moving symmetrically at $0.7 \mathrm{~m} / \mathrm{s}$ ) and then following locomotor adaptation on Day 2 (i.e. belts moving asymmetrically at $0.7 \mathrm{~m} / \mathrm{s}$ and $1.4 \mathrm{~m} / \mathrm{s}$ ). During training, participants were found to adapt their walking patterns at a behavioural level, which was associated with connectivity changes in the cerebellar-thalamic-cortical network (Mawase et al. 2017). Furthermore, their results suggest that recall and savings are associated with two separate neural networks (Mawase et al. 2017).

\section{Proprioceptive Recalibration}

Changes in the sensory system have been shown to accompany changes in the motor system. In fact, previous research has suggested that reach adaptation in response to altered visual feedback of the hand may arise due to changes in perceived felt hand position that occur in order to re-align one's felt hand position with the distorted visual feedback provided (Cressman \& Henriques 2010; Harris 1963, 1965; Redding \& Wallace 1988, 2002, 2003, 2006;

Salomonczyk et al. 2011). Shifts in felt hand position have been shown to arise after reach training with prisms and a visuomotor rotation (Cressman \& Henriques, 2009, 2010; Harris 1963, 1965; Zbib et al. 2016). Moreover, shifts in felt hand motion are observed after reaching in a velocity-dependent force-field (Ostry et al. 2010).

Changes in the sensory system were initially studied following reach training while wearing laterally displacing prisms (Craske \& Gregg, 1966; Harris 1963, 1965; Hay \& Pick, 


\section{LONG-TERM RETENTION OF PROPRIOCEPTIVE RECALIBRATION}

1966; Redding \& Wallace, 1998, 1993, 1996, 1997a, 1997b, 2001, 2002). In said experiments, participants were blindfolded following prism training and instructed to reach to a proprioceptive target that was defined as the participants' unadapted hand (Craske \& Gregg, 1966; Harris, 1965) or body midline (Harris, 1963; Hay \& Pick, 1966). Overall, participants felt their reaching hand had shifted position, such that the felt hand positions of participants who performed training with prisms were farther apart in comparison to those who performed reaches without prisms (Harris 1963, 1965). This led to the idea that changes in felt hand position arose following prism reaches such that proprioception was recalibrated. In more recent experiments, alignment tasks were used to measure sensory recalibration after having participants reach with a cursor that was translated from their actual hand position in a virtual reality environment (Simani et al. 2007; van Beers et al. 2002). Specifically, participants were instructed to align the fingertip of their unseen hand either with a visual target or with the other unseen hand (proprioceptive target) before and after reach training trials. They found changes in reaches to the proprioceptive target, indicating that proprioception had been recalibrated (Simani et al. 2007).

Recently, researchers stopped having participants make movements to assess changes in the sensory system, to ensure that these changes do not reflect motor adaptation. For example, Cressman and colleagues $(2009,2010)$ used a two-joint robot manipulandum to guide a participant's hand to different locations in the workspace. Participants were then asked to indicate the position of their hand (right or left) relative to a visual or proprioceptive marker (i.e. body midline) (Cressman \& Henriques, 2009, 2010; Zbib et al. 2016). The marker only appeared after the hand had been moved into position, thus ensuring that participants could not use the marker as a "reaching" target. A similar method was adopted by Ostry and colleagues to assess 


\section{LONG-TERM RETENTION OF PROPRIOCEPTIVE RECALIBRATION}

changes in hand motion following reaching in a velocity-dependent force field (Ostry et al. 2010; Mattar et al 2011, 2013).

The first experiment by Cressman and Henriques (2009) using this method to examine changes in felt hand position found a $6^{\circ}$ change in participants' estimates of felt hand position in the direction of the visual feedback provided. Notably, participants in the experiment were trained to reach with a gradually introduced cursor rotation of $30^{\circ} \mathrm{CW}$ relative to their actual hand position. As well, similar shifts in felt hand position have been shown to occur when the visuomotor distortion is introduced abruptly (Salomonczyk et al. 2012; Zbib et al. 2016), suggesting that the magnitude of the initial reach errors experienced does not influence proprioceptive recalibration. Finally, Salomonczyk et al. (2011) have shown that additional reach training trials within a single testing session (i.e. 3 blocks of 99 reach training trials) did not lead to increased proprioceptive recalibration. In fact, Zbib and colleagues (2016) have shown that proprioceptive recalibration does not increase significantly after 70 reach training trials.

\subsection{Time Course of Proprioceptive Recalibration}

Limited experiments have looked at the time course of sensory changes following reach training with a visuomotor distortion or in a velocity dependent force-field. Recently, Mattar et al. (2013) looked to determine when sensory changes, along with motor changes, arise following reaches in a velocity-dependent force field paradigm. They found that while motor changes occurred early (within 20 trials), sensory changes were slower to appear (appearing after 100 trials of force-field training). In a similar experiment by Zbib et al. (2016), the time course of sensory changes was also examined, but this time while training to reach with a visuomotor distortion, specifically a cursor that was rotated $30^{\circ} \mathrm{CW}$ relative to their hand. Reach errors and proprioceptive estimates of felt hand position were assessed at seven different times during 150 


\section{LONG-TERM RETENTION OF PROPRIOCEPTIVE RECALIBRATION}

rotated reach training trials. The results revealed a gradual shift in proprioceptive estimates throughout the rotated reach training trials, such that a significant change in estimates from baseline was not observed until after 70 reach training trials. The shift in proprioceptive recalibration was slower to arise compared to visuomotor adaptation, which arose quickly after 5 trials of training with the rotated cursor. Notably, Ruttle et al. (2016) observed early initial sensory changes after 6 rotated reach training trials. Interestingly, these sensory changes did not increase after the initial recalibration observed and were still small in magnitude compared to the motor changes observed during the reaching task. In conclusion, in both force-field and visuomotor distortion paradigms, proprioceptive changes appear to arise slowly during reach adaptation and follow a slowly increasing exponential curve (Matter et al. 2013; Zbib et al. 2016).

\subsection{Recall of Proprioceptive Recalibration}

As mentioned, experiments examining retention have shown long-term changes in the motor system; however, a similar investigation has not been done into potentially long-term changes in the sensory system. More specifically, it is important to highlight that changes in sense of felt limb motion or position have for the most part only been assessed 24 hours following initial training (Nourouzpour et al. 2015; Ostry et al. 2010). For example, Ostry et al. (2010) showed that changes in participants' sense of limb motion persisted 24 hours after reaching in a velocity dependent force-field. As well, Nourouzpour et al. (2015) recently found that changes in felt hand position persisted 24 hours after adapting to a gradually introduced visuomotor distortion, such that participants who showed proprioceptive recalibration immediately following training also showed significant retention of sensory changes $(72 \%$ of 


\section{LONG-TERM RETENTION OF PROPRIOCEPTIVE RECALIBRATION}

initial shift in hand position). From these experiments, it is evident that sensory changes are present up to at least 24 hours following training.

One experiment that investigated more long-term changes in the sensory system, is Hatada et al. (2006), where participants were required to perform reaching tasks to proprioceptive targets. Specifically, they showed that one session of prism adaptation training lasting 75 minutes generated long-lasting sensory changes. The sensory changes were assessed by having participants point straight-ahead in the dark during recall (i.e. aftereffects) sessions on 7 consecutive days. Immediately following testing, participants sensed their hand was shifted $\sim 34 \%$ of the distortion. This shift in hand position was absent 6 hours after reach training. However, two days after completing the initial reach training (Day 3), shifts in felt hand position were again present and reached their maximum, peaking at $38 \%$ of the distortion. The increased shift in felt hand position over subsequent days may stem from an adaptive component, characterized by a slow remapping of the sensorimotor system, instead of a conscious strategic adjustment component which quickly reduces errors (Hatada et al. 2006; Redding \& Wallace 1996, 2005).

As highlighted at the beginning of this section, experiments examining sensory retention following visuomotor adaptation are limited. However, there is evidence of long-term changes in the sensory system with training, specifically with regards to topographic representations in the primary somatosensory cortex (S1). For example, experiments have examined changes in the topographic representations of the hand following extended training in a frequencydiscrimination task and after behaviourally controlled tactile stimulation (Jenkins et al. 1990; Recanzone et al. 1992a; Recanzone et al. 1992b). Specifically, in the experiment by Recanzone et al. (1992b), the responses of cortical neurons evoked by cutaneous stimulation in adult owl 


\section{LONG-TERM RETENTION OF PROPRIOCEPTIVE RECALIBRATION}

monkeys following training in a tactile frequency-discrimination task were investigated. Animals performed 500-700 trials per day, resulting in 15-22 minutes of tactile simulation per session, with the number of sessions (1 per day) ranging from $58-131$. Researchers found that cortical representations of the trained hands, where large cutaneous receptive fields were stimulated, were substantially more complex topographically than those of the unstimulated hand (Recanzone et al. 1992a; Recanzone et al. 1992b). Similar results were observed when monkeys were taught to regulate their contact pressure and duration with a rotating disk in order to receive a food prize (Jenkins et al. 1990). Training lasted approximately 10 days with over 600 trials occurring within a 24 hour training period. These experiments demonstrate that long-term changes in the sensory system are possible with extended training.

\section{Proposed Research and Hypotheses}

The current research seeks to determine the ability of the sensory system to retain information over a long period of time (i.e. 5 days) in the form of recall and savings. As well, we ask whether additional training benefits short-term retention of proprioceptive recalibration (i.e. 24 hours). Two groups of participants adapted to a cursor rotated $30^{\circ} \mathrm{CW}$ relative to their hand on an initial testing day. Testing then took place on 4 consecutive days ( 5 testing days in total). Each day, participants completed no-cursor reaching trials to assess visuomotor adaptation and proprioceptive estimates to assess proprioceptive recalibration, relative to baseline measurements. Participants in the "Training group", completed additional reach training trials on each testing day. Participants in the "Non-Training group" did not complete these additional trials. In general, changes in the motor and sensory systems were analyzed over time during the first day of testing, while testing on days 2-5 included either testing for immediate retention in the form of recall (Non-Training group) or for immediate recall and savings when the 


\section{LONG-TERM RETENTION OF PROPRIOCEPTIVE RECALIBRATION}

perturbation was reintroduced (Training group). We hypothesized that on Day 1, proprioceptive estimates of hand position would be recalibrated following reach training trials with a rotated cursor. This sensory change was expected to arise slowly compared to visuomotor adaptation. We expected to observe recall of sensory (and motor) changes 24 hours after the initial training day and hypothesized that additional training would lead to greater recall and savings on subsequent testing days. These results would suggest that additional training, specifically on a consistent basis, has the ability to maintain and improve sensory performance as training progresses. For the Non-Training group, we expected that recall of proprioceptive recalibration would decrease over subsequent testing days in the absence of additional training. Despite the expected decrease in retention, we still expected to observe savings on Day 5 with respect to proprioceptive recalibration. These results would suggest that sensory changes, while limited, are still retained in the system even 5 days after initial training and imply that retention should be assessed in the form of recall and savings. 
LONG-TERM RETENTION OF PROPRIOCEPTIVE RECALIBRATION

\section{Chapter II: Research Article}

Long-term retention of proprioceptive recalibration

Stefan Maksimovic ${ }^{1} \&$ Erin K. Cressman ${ }^{1}$

${ }^{1}$ School of Human Kinetics, University of Ottawa, Ottawa, Canada

Keywords:

Proprioceptive recalibration

Recall

Savings

Visuomotor adaptation

Motor learning 


\title{
LONG-TERM RETENTION OF PROPRIOCEPTIVE RECALIBRATION
}

\begin{abstract}
Research has shown that reaching with altered visual feedback of the hand results in sensory changes, such that proprioceptive estimates of hand position are shifted in the direction of the visual feedback experienced (Cressman \& Henriques 2009). This experiment examined the ability of proprioceptive recalibration to be retained over an extended period of time (i.e. four days), and the benefits of additional training on retention in the form of recall and savings (i.e. faster re-learning on subsequent testing days). Twenty-four participants trained to reach to a target while seeing a cursor that was rotated $30^{\circ}$ clockwise relative to their hand on an initial day of testing. Half of the participants then completed additional reach training trials on 4 subsequent testing days (Training group), whereas the second half of participants did not complete additional training (Non-Training group). Participants provided estimates of their felt hand position on all 5 testing days to establish retention of proprioceptive recalibration. Results revealed that proprioceptive recalibration was recalled 24 hours after initial training and that there was no benefit of additional training. Retention in the form of savings was observed on all days for the Training group and on Day 5 in the Non-Training group. These results reveal that proprioceptive recalibration does not benefit from additional training but is retained in the form of recall and savings. Moreover, the different time scales (i.e. one day for recall versus four days for savings), suggest that distinct processes may underlie recall and savings of proprioceptive recalibration.
\end{abstract}




\section{LONG-TERM RETENTION OF PROPRIOCEPTIVE RECALIBRATION}

\section{Introduction}

When reaching to visual objects in one's environment, a series of sensory to motor transformations occurs in order to ensure that one successfully reaches to a desired object. Specifically, visual information related to the hand and target position and proprioceptive information regarding hand position are transformed into appropriate motor commands (Desmurget et al. 1998; Flanders et al. 1992; Jeannerod 1988). When reaching in a virtual reality environment with altered visual feedback of the hand, individuals use the altered visual feedback to rapidly reduce their initial reach errors in order for the visual representation of their hand to achieve the target; a process known as visuomotor adaptation (Cressman \& Henriques, 2009; Krakauer et al. 1999, 2005; Simani et al. 2007). After adapting their movements, individuals continue to reach with the updated movement pattern, even when visual feedback is removed. These reach errors, known as aftereffects, are evidence that persistent changes have occurred in the motor system (Baraduc \& Wolpert 2000; Buch et al. 2003; Cressman \& Henriques 2009, 2010; Cressman et al. 2010; Krakauer et al. 1999, 2000; Martin et al. 1996; Zbib et al. 2016).

In addition to changes in the motor system, proprioceptive recalibration has been shown to occur following reach training with misaligned visual feedback of the hand. Specifically, participants recalibrate their sense of felt hand position (i.e. proprioceptive recalibration), such that they feel their hand is shifted in the direction of the visual feedback provided (Cressman \& Henriques 2009; Zbib et al. 2016). This proprioceptive recalibration has been assessed in tasks in which the participant's hand is guided out passively to different locations in the workspace by a robot manipulandum and the participant indicates the position of their hand relative to a visual or proprioceptive reference marker (Cressman \& Henriques 2009), as well as in tasks requiring 


\section{LONG-TERM RETENTION OF PROPRIOCEPTIVE RECALIBRATION}

participants to actively reach to a proprioceptive target (Ruttle et al. 2016; Simani et al. 2007; van Beers et al. 2002).

Recently, Zbib and colleagues (2016) looked to determine how quickly proprioception was recalibrated when training to reach with a cursor that was rotated $30^{\circ}$ clockwise $(\mathrm{CW})$ relative to hand motion. They found that shifts in proprioceptive estimates took longer to arise in comparison to visuomotor adaptation as assessed through aftereffect trials. Specifically, Zbib et al. (2016) found that proprioception had shifted by $8.8^{\circ}$ ( $30 \%$ of the $30^{\circ}$ cursor distortion) relative to baseline after participants completed 70 rotated reach training trials, while the motor system showed evidence of adaptation equivalent to $16.9^{\circ}\left(\sim 56 \%\right.$ of the $30^{\circ}$ distortion) after only 5 reach training trials. Moreover, the shifts in proprioception observed after 70 trials did not increase significantly again with an additional 80 trials of training, such that proprioceptive recalibration plateaued at $8.8^{\circ}$ (or $30 \%$ of the $30^{\circ}$ distortion). On the other hand, the early reach errors observed plateaued after only 40 trials of training at $22.3^{\circ}$ (or $74.3 \%$ of the $30^{\circ}$ distortion). A more recent experiment by Ruttle and colleagues (2016), also showed greater visuomotor adaptation after only 6 reach training trials with visual feedback rotated relative to hand motion $\left(10.5^{\circ}\right.$ or $26.2 \%$ of the $40^{\circ}$ visuomotor rotation distortion) compared to proprioceptive recalibration following those same 6 training trials $\left(5.2^{\circ}\right.$ or $13 \%$ of the $40^{\circ}$ visuomotor rotation distortion). Like Zbib et al. (2016), proprioceptive recalibration did not continue to significantly increase over additional reach training trials, such that at the end of reach training, the extent of proprioceptive recalibration was much less than visuomotor adaptation. Interestingly, additional reach training sessions within a single testing day did not appear to lead to greater proprioceptive recalibration (Salomonczyk et al. 2011). Together, these results suggest that proprioceptive recalibration arises more slowly compared to visuomotor adaptation and that proprioception may 


\section{LONG-TERM RETENTION OF PROPRIOCEPTIVE RECALIBRATION}

only be able to be recalibrated up to a maximal point, well below the magnitude of visuomotor adaptation.

While the time course and magnitude of changes in the motor and sensory systems following training with distorted visual feedback of the hand have been shown to differ with initial testing, both show evidence of retention. Retention can be established by observing (1) aftereffects at the start of a second testing session, which we will refer to as recall (Mawase et. al 2017) and/or (2) savings within a second testing session, such that there is faster relearning upon exposure to a previously experienced environment compared to initial training performance (Krakaeur et al. 2005; Krakauer, 2009). Retention of visuomotor adaptation has been firmly established by observing the recall of aftereffects and savings at various times following training with the initial visuomotor rotation perturbation (e.g. 24 hours (Nourouzpour et al. 2015), 1-2 week(s) and 12 months (Yamamoto et al. 2006) post initial training). For example, Nourouzpour et al. (2015) found retention of visuomotor adaptation following reach training with a $40^{\circ}$ clockwise $(\mathrm{CW})$ visuomotor rotation, such that immediate aftereffects in the direction of the rotation were not different from those measured 24 hours later. Furthermore, results from Yamamoto et al. (2006) showed significant long-lasting changes in motor performance in the form of recall and suggested that even relatively short-term reach training with distorted visual feedback of the hand (e.g. 120 trials for humans \& 150 trials for monkeys) leads to long-term changes in the motor system $(1-2$ week(s) \& $>1$ year). With respect to savings of visuomotor adaptation, Klassen et al. (2005) observed that initial reaching errors decreased more rapidly 24 hours after initially reaching with a cursor rotated $30^{\circ} \mathrm{CCW}$ relative to the hand. Furthermore, experiment have shown similar results in follow-up testing sessions 48 hours (Caithness et. al. 2004) and 1 week (Krakauer et al. 2005) after initial training. Interestingly, it has recently been 


\section{LONG-TERM RETENTION OF PROPRIOCEPTIVE RECALIBRATION}

suggested that the neural circuitry underlying these two forms of retention may differ (Mawase et al. 2017).

To date, experiments examining retention of proprioceptive recalibration following reach training are limited. Specifically, experiments examining retention of proprioceptive recalibration following reach adaptation trials have focused on examining retention in the form of recall 24 hours after initial training in the form of aftereffects (Nourouzpour et al. 2015; Ostry et al. 2010), with no examination into potential savings. Within these previous experiments, Ostry et al. (2010) showed that changes in sense of limb motion persisted 24 hours after reaching in a velocity dependent force-field, while Nourouzpour et al. (2015) found that changes in felt hand position persisted 24 hours after adapting to a gradually introduced visuomotor rotation distortion, such that participants who showed proprioceptive recalibration immediately following training, also showed significant recall of sensory changes. Results from Hatada et al. (2006) suggested that longer-term retention of sensory changes (e.g. 7 days) may be possible, as they found continued changes in reaches to proprioceptive targets 7 days after participants reached to targets while wearing prisms, with the greatest recall occurring two days after participants completed initial training. However, these sensory changes were assessed with a reaching task and hence retention of visuomotor adaptation may have influenced their results.

The goal of the current experiment was to establish retention of proprioceptive recalibration over an extended period of time (4 days) in the form of recall and savings. As well, we looked to determine the benefits of additional training on short-term retention of proprioceptive recalibration. All of our participants trained to reach to targets when provided with rotated visual feedback of the hand (i.e., a $30^{\circ} \mathrm{CW}$ cursor rotation). Over the course of the reach training trials on an initial day of testing, we had participants reach to targets without 


\section{LONG-TERM RETENTION OF PROPRIOCEPTIVE RECALIBRATION}

visual feedback to assess the timeline of visuomotor adaptation in the form of aftereffects and estimate where they felt their hand was located in space to determine the timeline of proprioceptive recalibration. Following this initial day of testing, participants were divided into two groups: one with similar, additional, reach training across 4 consecutive days of testing (Training group) and another which did not perform the additional reach training trials (NonTraining group) on days 2 through 4 . On the $5^{\text {th }}$ day, all participants performed the reach training trials. We measured retention in the form of recall on 4 consecutive days of testing following the initial training day. As well, savings was assessed on each training day in the Training group and in the Non-Training group on Day 5. Similar to previous results (Nourouzpour et al. 2015), we hypothesized that all participants would recalibrate their sense of felt hand position on the first day of testing and recall these sensory changes 24 hours following initial training. Furthermore, we hypothesized that the Training group would exhibit greater recall of proprioceptive recalibration as training progressed on subsequent days, thus allowing for greater savings and potentially a greater magnitude of proprioceptive recalibration across the testing days as well. For the Non-Training group, we expected recall of proprioceptive recalibration would decrease across the next three testing days, though there might be evidence of savings on Day 5 , indicating the presence of two distinct neural networks underlying recall and savings of proprioceptive recalibration.

\section{Methods}

\subsection{Participants}

Twenty-four young adults ( 9 female, 15 male; mean age $=22.1$ years, $S D=2.3$ ) were recruited and took part in this experiment. Participants were divided into two groups: i) a Training group $(n=12)$ and ii) a Non-Training group $(n=12)$. All participants were deemed 


\section{LONG-TERM RETENTION OF PROPRIOCEPTIVE RECALIBRATION}

healthy following verbal screening for sensory, neurological or motor dysfunctions. Participants had normal or corrected-to-normal vision and prior to beginning the experiment all completed the modified version of the Edinburgh handedness inventory to confirm that they were right handed (mean handedness score $=95.4, S D=9.6$; Oldfield, 1971). Participants were naïve to our hypotheses and had never completed an experiment involving visuomotor adaptation to misaligned visual feedback of the hand. Each participant completed testing on 5 consecutive days, with each testing session lasting up to 1.5 hours. This experiment was approved by the University of Ottawa's research ethics board, and all participants provided informed consent before taking part in the experiment.

\subsection{Experimental Apparatus and Task}

Participants were seated in a height-adjustable chair in front of the experimental apparatus (see FIG. 1A). The height and distance of the chair from the apparatus was adjusted to ensure they could comfortably see and reach to the visual target. Measurements of the chair height and position from the apparatus were recorded to ensure that participants sat in a similar position on each testing day. Participants then grasped the vertical handle of a two-joint robot manipulandum (BKIN technologies) with their right hand, and made reaching movements to a visual target within a $70 \mathrm{~cm}$ by $36 \mathrm{~cm}$ workspace (see FIG. 1B). Approximately $20.5 \mathrm{~cm}$ above the robot handle was a reflective surface, that displayed targets that were projected from a downward facing monitor that was $20.5 \mathrm{~cm}$ above the reflective surface (EzSign model 47LD452B; refresh rate: $60 \mathrm{~Hz}$; LG, Seoul, South Korea; FIG. 1A). Thus, the targets appeared to lie in the same plane as the reaching hand.

To start a trial, the robot placed the participant's hand in the home position, which was aligned with their body midline and approximately $20 \mathrm{~cm}$ in front of their chest. The visual target 


\section{LONG-TERM RETENTION OF PROPRIOCEPTIVE RECALIBRATION}

(white circle; $1 \mathrm{~cm}$ in diameter) appeared $15 \mathrm{~cm}$ and $45^{\circ} \mathrm{CW}$ from the home position relative to straight ahead (see FIG. 1B). The lights in the room were dimmed and participants could not see their hands or arms, as a black cover was placed between their shoulders and the apparatus.

\subsection{Experimental Procedure}

Once a participant was comfortably seated, the task and procedures for the experimental session(s) were fully explained by the experimenter. The experiment took place over 5 consecutive days, with testing sessions separated by approximately 24 hours. All participants completed all tasks on the first testing day, which included 8 testing blocks divided into 2 sessions; the first block being completed in the first session and the seven following blocks being completed in the second session (as shown in FIG. 2). A break of 10 to 15 minutes was provided between the two sessions. Prior to the first session, participants began the experiment by performing a short practice block of 5 reaches and proprioceptive estimates (explained below) to familiarize themselves with the reaching task and the apparatus. The practice data were not analyzed. Within each block participants performed three experimental tasks, outlined in more detail below. The blocks contained 50 (Block 1), 5 (Block 2), 5 (Block 3), 10 (Block 4), 20 (Block 5), 30 (Block 6), 30 (Block 7) and 50 (Block 8) reach training trials. After completing each set of reach training trials, participants performed 6 no-cursor reaches followed by 50 proprioceptive estimate trials and, finally, 6 more no-cursor reaches. For the group receiving additional training (Training group), 8 testing blocks were also completed on Day's two to five. However, on these days, the blocks contained 0 (Block 1), 5 (Block 2), 5 (Block 3), 10 (Block 4), 20 (Block 5), 30 (Block 6), 30 (Block 7) and 50 (Block 8) reach training trials (see FIG. 2). The first set of reaching trials did not include any reach training trials (i.e. 0), as we wanted to measure retention of the motor and sensory systems following training from previous day(s) in 


\section{LONG-TERM RETENTION OF PROPRIOCEPTIVE RECALIBRATION}

the form of recall (i.e. aftereffects). Similar to the first day, 6 no-cursor reaching trials, 50 proprioceptive estimates and 6 no-cursor reaching trials followed each of the sets of reach training trials and there was a break of 10 - 15 minutes following the first block. For the remaining participants (Non-Training group), only the first testing block was performed on Day's two to four. Specifically, the single block they performed contained 0 (Block 1) reach training trials along with 6 no-cursor reaching trials, 50 proprioceptive estimates trials and, again, 6 no-cursor reaching trials. However, on Day 5, the Non-Training group performed the same 8 blocks as the Training group.

\subsubsection{Reach Training Task}

During the reach training trials, participants reached to the single visual target. Participants were instructed to grasp the robot handle with a comfortable, but firm grip. Hand position was maintained at the home position for $500 \mathrm{~ms}$, after which the visual target appeared $15 \mathrm{~cm}$ and $45^{\circ}$ CW from the home position relative to straight ahead (shown in FIG. 1B). The home position was only displayed at the beginning of the reach training trials (white circle, $1 \mathrm{~cm}$ in diameter). Participants were instructed to reach as quickly and accurately as possible to the target while holding the robot handle. Visual feedback regarding the position of the reaching hand was provided in the form of a green cursor $(1 \mathrm{~cm}$ in diameter) once the robot handle had moved $7 \mathrm{~cm}$ towards the target, in correspondence to the position where participants typically achieved peak velocity. During Block 1, the cursor was aligned with the actual position of the participant's hand; therefore, if their hand went directly to the target, so did the cursor. During Blocks 2 - 8 , the cursor was rotated $30^{\circ} \mathrm{CW}$ relative to their hand. Once the green cursor was within $0.5 \mathrm{~cm}$ of the target's center, the reach was deemed complete. Upon completion, the target and cursor were removed and the robot was locked to a grooved path which moved the participant's hand back to 


\section{LONG-TERM RETENTION OF PROPRIOCEPTIVE RECALIBRATION}

the home position by way of a linear route without visual feedback. If participants attempted to move outside the grooved path, a resistance force (proportional to the depth of penetration with a stiffness of $2 \mathrm{~N} / \mathrm{mm}$ and a viscous damping of $5 \mathrm{~N} /(\mathrm{mm} / \mathrm{s})$ ) was generated perpendicular to the grooved wall to maintain the linearity of the movement. The position of the robot manipulandum was recorded at all times during all reaching tasks at a sampling rate of $1000 \mathrm{~Hz}$ and a spatial accuracy of $0.1 \mathrm{~mm}$.

\subsubsection{No-Cursor Reaches}

The no-cursor reaching trials were used to examine adaptation and recall in the motor system. Again, participants were instructed to grasp the robot handle with a comfortable, but firm grip. Hand position was maintained at the home position for $500 \mathrm{~ms}$, after which the visual target appeared. Participants were instructed to reach as quickly and accurately as possible to the target while holding the robot handle. During this task no visual feedback (i.e. no cursor) was displayed to participants. After completing their reach, and maintaining a final hand position for $1000 \mathrm{~ms}$, the visual target disappeared, signaling trial completion. Finally, the participant's hand was guided back to the home position by a linear grooved path.

\subsubsection{Proprioceptive Estimate Task}

The proprioceptive estimate trials were utilized to establish the position participants perceived their unseen hand to be aligned with a visual reference marker. Participants began by grasping the robot handle at the home position. On these trials the home position was displayed as a white circle $(1 \mathrm{~cm}$ in diameter) directly above the robot handle for $500 \mathrm{~ms}$. Following this, the home position was removed and the participant's hand was moved passively by the robot to a final position. Once the hand arrived at its final position (i.e. 1 second later), a visual reference marker (yellow circle; $1 \mathrm{~cm}$ in diameter) appeared $15 \mathrm{~cm}$ and $45^{\circ} \mathrm{CW}$ from the home position 


\section{LONG-TERM RETENTION OF PROPRIOCEPTIVE RECALIBRATION}

relative to straight ahead (i.e. the same position as the visual reaching target; see FIG. 1C).

Participants were instructed to make a two-alternative forced-choice judgment about the position of their hand, specifying whether they felt their unseen hand was to the left or the right of the visual reference marker. Participants' hand position relative to the reference marker was adjusted over the course of proprioceptive estimate trials, in each testing block, using an adaptive staircase algorithm (Cressman \& Henriques 2009, 2010; Kesten 1958; Treutwein 1995). As in Cressman and Henriques (2009), there were two staircases for the visual reference marker, with one starting $20^{\circ}$ left $(\mathrm{CCW})$ and the other $20^{\circ}$ right $(\mathrm{CW})$ of the reference marker (see FIG. 3A). Adjustments to each staircase were dependent on a participant's pattern of responses, such that the differences between hand locations in subsequent trials decreased each time the participant reversed his or her response pattern from left to right or from right to left within the staircase. This guaranteed that participants were tested more frequently at positions closer to the position they perceived their hand to be at the reference marker. If participants responded consistently, the two staircases converged towards a position at which participants had an equal probability of reporting left or right and perceived their hand to be aligned with the reference marker (see FIG. 3B). No time constraints were put on participants, as they were encouraged to take their time before verbally giving an answer to the experimenter during each proprioceptive estimation trial. Upon completion of the trial, the reference marker disappeared and the robot moved the hand back to the home position (1 second movement duration).

\subsection{Data Reduction and Analyses}

\subsubsection{Proprioceptive Estimates of Hand Position}

In order to establish if participants shifted their felt hand location during reach training trials and if this changed across days, we first determined the locations that participants felt their 


\section{LONG-TERM RETENTION OF PROPRIOCEPTIVE RECALIBRATION}

hands were aligned with the visual reference marker. This was done by fitting a logistic function to each participant's responses during the proprioceptive estimate trials completed within each block and calculating their bias (i.e. the point at which participants responded left and right $50 \%$ of the time). In addition, participants' precision for each bias was determined by calculating their uncertainty range (i.e. the difference between the values at which the probability of responding left was $25 \%$ and $75 \%$ ) (see FIG. 3B).

\subsubsection{Reach Errors}

Each reaching trial was examined; this included both reach training trials with the cursor and no-cursor reach trials. To begin, custom written programs in MATLAB were used to select the start and endpoints of all reaching trials using a velocity criteria (velocity first increased above or decreased below $0.01 \mathrm{~m} / \mathrm{s}$ for $50 \mathrm{~ms}$ respectively). In order to establish whether participants adapted their reaches and retained this information across days after training with a misaligned cursor, we first examined the no-cursor reaching trials completed before and after proprioceptive estimation trials on each testing day. Angular reach errors at both peak velocity (PV) and movement endpoint (EP) in the no-cursor reaching trials were determined. Angular errors at PV were defined as the angular difference between a movement vector (from the home position to peak velocity) and a reference vector (joining the home position and the target). EP angular errors were defined as the angular deviation between a movement vector (from the home position to the reach endpoint) and a reference vector (from the home position to the target). Reaching errors (PV and EP) were averaged over the 6 trials for each participant for each set of no-cursor reaches (i.e. trials completed at Time 1 or Time 2 within each block). For the reach training trials, we determined angular reach errors at peak velocity (PV) only, as participants had to land on the target for the trial to be considered complete. 


\section{LONG-TERM RETENTION OF PROPRIOCEPTIVE RECALIBRATION}

\subsubsection{Analyses of Proprioceptive Estimates}

The first question we examined was: How long is proprioceptive recalibration retained after reach training with distorted visual feedback of the hand? In order to answer this question, we first established that participants in both groups recalibrated their sense of felt hand position on Day 1 by comparing proprioceptive biases across Blocks on Day 1 using a 2 Group (Training vs. Non-Training) x 8 Block mixed analysis of variance (ANOVA) with repeated measures (RM) on the second factor. Once we established that proprioception was recalibrated for the two Groups, a 2 Group (Training vs. Non-Training) x 5 Day (Day 1 - 5) mixed ANOVA with RM on the last factor was performed on proprioceptive biases achieved in Block 1 across the 5 testing days to establish if retention in the form of recall was seen across testing days. For the analyses above, and all analyses discussed below, differences with a probability of less than 0.05 were considered significant and Bonferroni post-hoc tests corrected for multiple comparisons were administered to find the locus of these differences for all pre-planned comparisons.

Following this analysis, we looked to determine if retention in the form of savings was present for the Non-Training group on Day 5 (i.e. if proprioception was recalibrated faster on Day 5 compared to Day 1). To examine savings of proprioceptive recalibration, we first compared rates of change of proprioceptive biases across blocks on Days 1 and 5. Specifically, we fit exponential curves to the mean performance data across participants on each of the testing days using the curve fitting toolbox in MATLAB. The curves took the form of the following exponential function as used by Matter et al. (2013) and Zbib et al (2016):

$$
\hat{y}=a *\left[1-(1-b)^{x}+c\right]
$$

such that $y$ represents angular bias, $x$ represents the number of reach training trials completed just prior to the proprioceptive estimates, $a$ represents the scale of the change, $b$ represents the rate of 


\section{LONG-TERM RETENTION OF PROPRIOCEPTIVE RECALIBRATION}

change and $c$ represents the vertical offset of the function. Rates of change were determined to be significantly different if their $95 \%$ confidence intervals did not overlap. Furthermore, we used more traditional methods of examining savings (Krakauer et al. 2005), by (i) comparing when changes in proprioceptive biases plateaued (i.e. changes were no longer significant when compared to performance in the final block (Block 8)) between the 2 days using Bonferroni posthoc tests of a 2 Day (Day 1 vs. Day 5) x 8 Block RM-ANOVA and (ii) comparing the average performance over Blocks 2 - 3 on Day 1 versus Day 5 using a t-test.

Next we examined our second question: Does additional training benefit short-term (e.g. 24 hour) retention of proprioceptive recalibration? The results of the 2 Group (Training vs. Non-Training) x 5 Day (Day 1 - 5) mixed ANOVA described above which compared proprioceptive biases in Block 1 across the 5 testing days provided insight into potential changes in recall with extra training. With respect to savings (in the Training group), we first confirmed our observation that performance on Days 2 through Day 5 did not differ for the Training group by performing a 4 Day (Days $2,3,4,5)$ x 8 Block RM-ANOVA. ANOVA revealed no significant effect of Day $\left[F_{(3,33)}=.879, p=0.462\right]$ or interaction of Day and Block $\left[F_{(21,231)}=\right.$ $1.159, p=0.290$ ], establishing that performance was similar across these additional testing days. Thus, we collapsed the data over the 4 days by averaging performance across Days 2 through 5 for each participant for each block and compared rates of change between Day 1 and average proprioceptive biases on Day 2 - 5. Similar to above, we compared when proprioceptive biases plateaued between the 2 days using Bonferroni post-hoc tests of a 2 Day (Day 1 vs. average proprioceptive biases on Day 2 - 5) x 8 Block RM-ANOVA and compared the average performance over Blocks 2 - 3 on Day 1 versus Day 2 - 5 by using a t-test. 


\section{LONG-TERM RETENTION OF PROPRIOCEPTIVE RECALIBRATION}

\subsubsection{Analyses of Uncertainty Ranges}

Similar to the analyses of biases described above, we first confirmed our observation that uncertainty ranges on Days 2 through Day 5 did not differ across days for the Training group by performing a 4 Day (Days 2, 3, 4, 5) x 8 Block RM-ANOVA. ANOVA revealed no significant effect of Day $\left[F_{(3,33)}=0.879, p=0.462\right]$, nor significant Day x Block interaction $\left[F_{(21,231)}=\right.$ $1.159, p=0.290]$. Thus, uncertainty ranges were collapsed across Days 2 through 5 for the Training group and analyzed in a 2 Group (Non-Training vs. Training) x 2 Day (Day 1 vs. Day 5 or average uncertainty ranges on Days 2-5) x 8 Block mixed ANOVA with RM on the last two factors.

\subsubsection{Analyses of PV and EP Reach Errors}

While the primary focus of the research undertaken was to establish retention of proprioceptive recalibration, we examined reaching performance in the no-cursor reaches in a similar manner to determine recall and savings of visuomotor adaptation. First, we looked for differences in our two error measures (PV vs. EP) by comparing the sets of no-cursor reaches at Time 1 and Time 2 on Day 1 using a 2 Group (Non-Training vs. Training) x 2 Angular Error Type (Endpoint vs. Peak Velocity) x 2 Time (Time 1 vs. Time 2) x 8 Block mixed ANOVA with RM on the last two factors. Results revealed that average errors in the no-cursor trials at EP and PV followed similar trends across blocks, such that the ANOVA revealed no significant effect of Angular Error Type $\left[F_{(1,44)}=0.002, p>0.968\right]$ and no significant interactions involving the factor of Angular Type (all $p>0.061$ ). Thus, the remaining analyses on the no-cursor reaches examined reaching errors only at movement EP, which correspond to similar locations as the proprioceptive biases and was done by Zbib et al. (2016). 


\section{LONG-TERM RETENTION OF PROPRIOCEPTIVE RECALIBRATION}

Using EP error data, we looked to determine if the two Groups adapted their reaches on Day 1 using a 2 Group (Non-Training vs. Training) x 2 Time (Time 1 vs. Time 2) x 8 Block mixed ANOVA with RM on the last two factors. Once we established that reaches were adapted for the two groups on the first testing day, we then looked at retention in the form of recall by comparing reaching errors in Block 1 using a 2 Group (Training vs. Non-Training) x 2 Time (Time 1 vs. Time 2) x 5 Day (Day 1 - 5) mixed ANOVA with RM on the last two factors. Savings was assessed by comparing rates of change of EP reach errors across blocks on Days 1 and 5 in the Non-Training group. Notably, examination of savings at Time 1 was omitted as changes in reaches for both groups leveled off within 5 reach training trails, therefore only Time 2 was examined. As well, we compared when motor changes plateaued between the 2 Days at Time 2 using Bonferroni post-hoc tests of a 2 Day (Day 1 vs. Day 5) x 8 Block RM-ANOVA and compared average performance over Blocks 2 - 3 on Day 1 versus Day 5 at Time 2 using a paired sample t-test.

The influence of additional training was assessed in the Training Group by first examining performance across the additional days of testing using a 3 Day (Day 3 - 5) x 8 Block RMANOVA. Given that reaching performance was similar on testing Days 3 through 5, as indicated by no effect of Day $\left[F_{(2,22)}=.996, p=0.385\right]$ and no interaction of Day x Block $\left[F_{(7,154)}=1.698\right.$, $p=0.145]$, participant data were collapsed across the final three testing days. Savings was then assessed at Time 2 by comparing rates of change between Day 1, Day 2 and average visuomotor adaptation across Days 3 - 5. Similar to above, we compared when motor changes plateaued at Time 2 over the three testing days using Bonferroni post-hoc tests of a 3 Day (Day 1 vs. Day 2 vs. average reach errors on Day 3 - 5) x 8 Block RM-ANOVA and compared average reach errors over Blocks 2 - 3 on Days 1, 2 and Day 3 - 5 at Time 2 using a 3 Day RM-ANOVA. 


\section{LONG-TERM RETENTION OF PROPRIOCEPTIVE RECALIBRATION}

\subsubsection{Analyses of Reach Training}

In order to determine if there was evidence of savings in the reach training trials, as typically done (Krakauer et al. 2005), we looked to compare average PV angular errors on the second and third reach training trials across days. Specifically, we first established that there was no difference in PV angular errors for the Training group across Days 2 through 5 using a 4 Day (Day 2, 3, 4, 5) RM-ANOVA, as indicated by no effect of Day $\left[F_{(3,33)}=2.728, p=0.110\right]$. We then performed a 2 Group (Non-Training vs. Training) x 2 Day (Day 1 vs. Day 5) mixed ANOVA with RM on the last factor, to determine if participants exhibited evidence of savings during the reach training trials.

\subsubsection{Post-Experiment Questioning}

Upon completion of the five days of testing, participants were asked whether they thought the reaching environment had changed at any point during training. Participants who responded they experienced changes were further asked if they could explain these changes and if they developed a strategy to adapt to them during training. When probed at the end of the experiment, all of our participants reported that they thought the reaching environment had maybe changed, but were unable to describe the distortion and when it was introduced. Thus, no strategies were reported to have been used. As a result, all participants were classified to have remained unaware of the distortion (i.e. that the cursor that was rotated $30^{\circ} \mathrm{CW}$ relative to their hand)

\section{Results}

\subsection{Proprioceptive Recalibration and Retention}

\subsubsection{Proprioceptive Recalibration}

Estimates of hand position were analyzed to determine the time course of proprioceptive recalibration on the initial testing day. Following training with an aligned cursor on Day 1, 


\section{LONG-TERM RETENTION OF PROPRIOCEPTIVE RECALIBRATION}

proprioceptive estimation trials revealed an initial bias, such that participants in both the NonTraining and Training groups felt their hands were at the reference marker when they were shifted left of it by approximately $5.2^{\circ}(S D=3.42)$ and $7.0^{\circ}(S D=5.6)$, respectively. As presented in FIG. 4A, proprioceptive estimates of hand position shifted further left of these baseline estimates while participants completed the reach training trials with rotated visual feedback of their hand. Specifically, estimates shifted by $11.7^{\circ}$ and $13.3^{\circ}$ for the Non-Training and Training groups after 150 reach training trials performed on the initial day of testing (see FIG. 4C). In accordance with these observations, ANOVA revealed a significant effect of Block $\left[F_{(7,154)}=85.77, p<0.001\right]$, indicating that the position at which participants felt their hand was aligned with the visual reference marker changed throughout reach training. There was no main effect of Group $\left[F_{(3 ., 22)}=1.733, p>0.202\right]$ and no significant Group x Block interaction $\left[F_{(7,154)}\right.$ $=0.423, p>0.786]$.

From FIG. 4A, it is evident that proprioceptive recalibration was gradual on Day 1. Post hoc analyses revealed that proprioceptive biases significantly differed from baseline performance after 5 reach training trials, or following Block 2 for all participants (Mean shift from Block 1 to $\left.2=3.891^{\circ}, S D=1.47 ; p<0.01\right)$. Moreover, biases continued to differ between each subsequent block until Block 5 (e.g. biases in Block 3 were different from Block 2). Following Block 5 (or after participants had completed 40 reach training trials) proprioceptive biases did not differ from those achieved in subsequent blocks (i.e. proprioceptive biases did not differ significantly from Block 5 to Block $8 ; p>0.26$ ), indicating a leveling off or plateau in proprioceptive recalibration. These results confirm that proprioceptive recalibration occurred on Day 1.

On Day 1, participants' levels of precision in estimating the location of their unseen hand were comparable between Groups $\left(F_{(1,22)}=.204, p=0.656\right)$ and across all blocks of reach 


\section{LONG-TERM RETENTION OF PROPRIOCEPTIVE RECALIBRATION}

training trials $\left[F_{(7,154)}=1.915, p>0.071\right]$. The average uncertainty range for both groups across all blocks was $7.9^{\circ}(S D=4.42)$. ANOVA revealed a main effect of Day $\left[F_{(1,22)}=16.457, p<\right.$ 0.001], but no Group x Day interaction $\left[F_{(1,22)}=0.450, p>0.509\right]$, such that uncertainty ranges were slightly greater on subsequent days of testing compared to the initial testing day (Mean Uncertainty Range $=10.6^{\circ}, S D=6.5$ ).

\subsubsection{Retention of Proprioceptive Recalibration (Recall)}

On Day 2, both groups showed evidence of recall of proprioceptive recalibration following initial training on Day 1 (see FIG. 5A). The Non-Training group retained approximately $4.1^{\circ}(S D$ $=3.71)$ of their initial recalibration $\left(11.7^{\circ}\right)$ achieved on Day 1 ( $35 \%$ of initial recalibration). Similarly, the Training group retained approximately $5.0^{\circ}(S D=3.92)$ of their initial recalibration $\left(13.3^{\circ}\right)$ achieved on Day 1 ( $\sim 38 \%$ of initial recalibration). From FIG. 5A, we see that recall of proprioceptive recalibration then differed between the two groups on testing Days 3 through 5. Specifically, in the Non-Training group, recall of proprioceptive recalibration dropped down to near baseline levels without additional training $\left(\sim 0^{\circ}\right)$, whereas, recall in the Training group was maintained at approximately $5.5^{\circ}$. In accordance with these observations, ANOVA revealed a significant effect of Day $\left[F_{(4,88)}=8.598, p<0.001\right]$ and a significant Group x Day interaction $\left[F_{(4,88)}=5.389, p<0.001\right]$. Post-hoc analyses revealed that proprioceptive estimates on Day 2 differed from Day 1 for both groups (Non-Training: $p<0.016 \&$ Training: $p<0.002$ ), confirming recall of proprioceptive recalibration was observed 24 hours following initial training. However, for the Non-Training group, performance in Block 1 on Days 3, 4 and 5 returned to baseline levels achieved on Day 1 (all $p>0.99$ ). With respect to the Training group, performance in Block 1 did not differ on Days 2, 3, 4, or 5 (all $p>0.99$ ), which differed from Day 1 (all $p<0.012$ ). These results indicate that recall of proprioceptive recalibration was not 


\section{LONG-TERM RETENTION OF PROPRIOCEPTIVE RECALIBRATION}

observed 48 hours following initial training and that additional training did not lead to increased recall 24 hours later.

\subsubsection{Retention of Proprioceptive Recalibration (Savings)}

Changes in proprioceptive biases over time and across days are shown by the exponential curves fitted to the data for both groups, as seen in Figures $6 \mathrm{~A}$ and $6 \mathrm{~B}$. These changes are reflected in the associated rates of change (or slopes) illustrated in Figures 7A and 7B. Upon comparing rates of change on Day 1 and 5 in the Non-Training group, results were deemed significant, as the $95 \%$ confidence intervals did not overlap. Specifically, the rate of change for Day 1 was 0.06 (with the $95 \%$ confidence interval ranging from 0.04 to 0.08 and a $r^{2}$ value indicating that the curve accounted for $98.64 \%$ of the variance of the curve) and on Day 5 was 0.10 (with the $95 \%$ confidence interval ranging from 0.08 to 0.12 and a $r^{2}$ value indicating that the curve accounted for $99.24 \%$ of the variance of the curve). As well, proprioceptive recalibration reached a plateau by Block 4 on Day 5 compared to Block 5 on Day 1 (which corresponds to an extra 20 reach training trials). Finally, proprioceptive biases were significantly greater earlier on in Blocks 2 - 3 on Day 5 (mean bias $=11.96^{\circ}, S D=5.52$ ) compared to Day 1 $\left(\right.$ mean bias $\left.=9.83^{\circ}, S D=3.79\right)$, as confirmed by a paired sample t-test; $t(11)=2.213, p=0.049$ (see FIG. 8A).

For the Training group, there was great overlap with respect to rates of change of proprioceptive biases on the testing days [Day 1 rate of change $=0.06$, with the $95 \%$ confidence interval ranging from 0.04 to 0.09 and a $r^{2}$ value indicating that the curve accounted for $98.23 \%$ of the variance of the curve; Day $2-5=$ rate of change $=0.11$, with the $95 \%$ confidence interval ranging from 0.07 to 0.16 and a $r^{2}$ value indicating that the curve accounted for $97.75 \%$ of the variance of the curve]. While these data suggest the absence of savings with respect to 


\section{LONG-TERM RETENTION OF PROPRIOCEPTIVE RECALIBRATION}

proprioceptive recalibration, there is the confound that participants' initial biases differed between testing days, as illustrated in FIG. 6B. In accordance with this observation, our secondary analyses revealed evidence of savings. Specifically, proprioceptive recalibration reached a plateau in Block 3 on Day 2 - 5 compared to Block 5 on Day 1 (which corresponds to an extra 30 reach training trials). A paired-sample t-test further confirmed significantly greater proprioceptive recalibration earlier on in Day $2-5$ (mean bias $\left.=16.76^{\circ}, S D=6.69\right)$ compared to Day $1\left(\right.$ mean bias $\left.=12.99^{\circ}, S D=6.34\right), t(11)=3.001, p=0.011($ see FIG. $8 \mathrm{~A})$.

\subsection{Visuomotor Adaptation and Retention}

\subsubsection{Visuomotor Adaptation}

Mean angular errors at movement endpoint (EP) in the no-cursor reaching trials are displayed in FIG. 4B, both before and after the proprioceptive estimation trials (i.e. at Time 1 and Time 2). From FIG. 4B, we see that participants reached such that their hand was to the left of the target after training with an aligned cursor (Non-Training group EP reach error $=0.65^{\circ}$, $S D=2.66^{\circ}$ and Training group EP reach error $\left.=-1.626^{\circ}, S D=3.12^{\circ}\right)$. During rotated reach training, reach errors increased rapidly as participants aimed significantly more to the left of the target. More specifically, the average change in EP angular error after just 5 rotated reach training trials relative to baseline at Time 1 was $21.8^{\circ}(S D=4.93)$ for the Non-Training Group and $25.1^{\circ}(S D=3.99)$ for the Training group, while at Time 2 it was $10.35^{\circ}(S D=5.15)$ for the Non-Training group and $10.57^{\circ}(S D=4.28)$ for the Training group. Furthermore, the average change in EP angular error after 150 rotated reach training trials relative to baseline at Time 1 was $24.3^{\circ}(S D=4.93)$ for the Non-Training group and $28.2^{\circ}(S D=4.42)$ for the Training group, while at Time 2 it was $15.4^{\circ}(S D=6.83)$ for the Non-Training group and $20.1^{\circ}(S D=5.27)$ for the Training group, as presented in FIG. 1D. ANOVA revealed a main effect of Block $\left[F_{(7,154)}=\right.$ 


\section{LONG-TERM RETENTION OF PROPRIOCEPTIVE RECALIBRATION}

170.887, $p<0.001]$ and Time $\left[F_{(1,22)}=49.813, p<0.001\right]$ and significant Block $\mathrm{x}$ Time interaction $\left[F_{(7,154)}=27.987, p<0.001\right]$. There was no main effect of Group $\left[F_{(1,22)}=.671, p>\right.$ 0.42] and no significant interactions with respect to Group $\mathrm{x}$ Block $\left[F_{(1,154)}=3.048, p=0.095\right]$, Group x Time $\left[F_{(1,22)}=1.481, p=0.237\right]$ or Group $x$ Block $x$ Time $\left[F_{(7,154)}=1.607, p=0.137\right]$. Changes in reaches arose rapidly in both groups, and differed between Time 1 and Time 2 for both groups, as indicated by the Block x Time interaction. The time course of visuomotor adaptation at Time 1 showed a great initial increase which plateaued at Block 2 (i.e., after only 5 reach training trials), such that there were no further significant changes across consecutive blocks [ $p=0.137$ between Block 2 and 3]. The time course of visuomotor adaptation at Time 2 showed a smaller initial increase, and a more gradual increase until a leveling off occurred at Block 5 (i.e., after 40 reach training trials), such that there were no further significant changes across consecutive blocks [ $p=0.062$ between Block 5 and 6].

\subsubsection{Retention of Visuomotor Adaptation (Recall of Aftereffects)}

On Day 2, we observed that both groups showed evidence of retention in the form of recall of aftereffects following initial training on Day 1 (see FIG. 5B for Time 1 and FIG. 5C for Time 2). Returning to the lab on Day 2, participants in the Non-Training group reached with errors of approximately $7^{\circ}(S D=3.87)$ in Block 1 at Time 1 relative to baseline performance on Day 1 ( $\sim 28 \%$ of initial visuomotor adaptation), while at Time 2 they reached with errors of $6.2^{\circ}(S D=$

4.82) ( $40 \%$ of initial visuomotor adaptation). The Training group showed similar recall at Time 1 , with reach errors of $11.3^{\circ}(S D=4.94)$ relative to baseline performance on Day $1(39.5 \%$ of initial adaptation), while at Time 2 they reached with errors of $12.2^{\circ}(S D=5.61)(\sim 56 \%$ of initial adaptation). In accordance with these observations ANOVA revealed a significant main effect of Day $\left[F_{(4,88)}=68.501, p<0.001\right]$, and post hoc analyses indicated that performance in Block 1 on 


\section{LONG-TERM RETENTION OF PROPRIOCEPTIVE RECALIBRATION}

Day 2 differed from Block 1 on Day 1 for the Non-Training [Time 1: $p<0.001$, Time 2: $p<$ 0.032] and Training group [Time 1: $p<0.001$, Time 2: $p<0.001]$.

From FIG. 5B, we see that recall of visuomotor adaptation remained consistent across the next three testing days in the Non-Training group at Time $1\left(\right.$ mean performance $\left.=6.97^{\circ}\right)$ and at Time $2\left(\right.$ mean performance $\left.=3.59^{\circ}\right)$, with recall observed at Time 2 less than at Time 1 . On the other hand, for the Training group at Time 1, recall observed on Day 3 was slightly greater than that observed on Day 2 at Time 1, suggesting greater recall with additional training. In accordance with the observed differences in recall between the two groups and the two times, ANOVA revealed a significant main effect of Time $\left[F_{(1,22)}=20.946, p<0.001\right]$; and significant interactions of Group x Day $\left[F_{(4,88)}=20.759, p<0.001\right]$ and Day and Time $\left[F_{(4,88)}=9.369, p<\right.$ 0.001]. Specifically, post hoc analyses confirmed that at Time 1 performance on Day 2 was not different from Days 3 through 5 in the Non-Training group (all $p>0.244$ ), while reaching errors were greater on Day 3 vs. Day 2 for the Training group $(p<0.001)$. Also, reaching errors did not differ at Time 1 for the Training group between Days 3 through 5 (all $p>0.265$ ). With respect to Time 2 (see FIG. 5C), reaching errors did not differ between Days 2 through 5 for both groups (all $p>0.083$ ). These results provide evidence of retention in the motor system in the form of recall for up to 96 hours following initial training and suggest that additional training may lead to increased recall across testing days when assessed immediately (Time 1), as opposed to following a 5-minute break (Time 2).

\subsubsection{Retention of Visuomotor Adaptation (Savings)}

Changes in visuomotor adaptation over time and across days are shown by the exponential curves fitted to the data for both groups, as seen in Figures 6A and 6B. These changes are reflected in the associated rates of change (or slopes) illustrated in Figures 7A and 7B. Upon 


\section{LONG-TERM RETENTION OF PROPRIOCEPTIVE RECALIBRATION}

comparing rates of change at Time 2 on Day 1 and Day 5 in the Non-Training group, we found that the rates of change overlapped and thus were not deemed significantly different.

Specifically, the rate of change for Day 1 was 0.187 (with the $95 \%$ confidence interval ranging from 0.048 to 0.32 and a $r^{2}$ value indicating that the curve accounted for $92.10 \%$ of the variance of the curve) and on Day 5 was 0.104 (with the $95 \%$ confidence interval ranging from 0.08 to 0.12 and a $r^{2}$ value indicating that the curve accounted for $99.34 \%$ of the variance of the curve) (see FIG. 7A), suggesting a lack of savings, despite the evidence of slight recall. Moreover, visuomotor adaptation leveled off by Block 4 on both Day 1 and Day 5. Similarly, no significant difference between the average performance between Blocks 2 - 3 on these two days was found $\left(\right.$ mean reach errors Day $1=14.78^{\circ}, S D=7.71$; mean reach errors Day $5=15.65^{\circ}, S D=7.32$; $t(11)=0.413, p=0.687)($ see FIG. 8B)

For the Training group, the rates of change at Time 2 also overlapped across testing days suggesting a lack of savings. Specifically, the rate of change for Day 1 was 0.395 (with the $95 \%$ confidence interval ranging from 0.139 to 0.65 and a $r^{2}$ value indicating that the curve accounted for $97.85 \%$ of the variance of the curve), on Day 2 was 0.273 (with the $95 \%$ confidence interval ranging from 0.143 to 0.404 and a $r^{2}$ value indicating that the curve accounted for $97.75 \%$ of the variance of the curve) and on Day 3 - 5 was 0.2 (with the $95 \%$ confidence interval ranging from 0.1263 to 0.29 and a $r^{2}$ value indicating that the curve accounted for $97.72 \%$ of the variance of the curve (see FIG. 7B). However, as mentioned above, this measure of savings was confounded by the fact that participants begin the testing sessions on Days 2 through 5 with greater reach errors compared to Day 1, as illustrated in FIG. 6B. Our secondary analyses revealed evidence of savings, whereby visuomotor adaptation plateaued in Block 4 on Day 2 and Block 3 on Day 3 - 5 compared to Block 5 on Day 1. Moreover, a RM-ANOVA revealed a main effect of Day $\left[F_{(2,22)}\right.$ 


\section{LONG-TERM RETENTION OF PROPRIOCEPTIVE RECALIBRATION}

$=25.512, p=0.0001]$ and post-hoc analyses confirmed greater average visuomotor adaptation across Blocks 2 - 3 on Day 2 (mean reach errors $\left.=22.34^{\circ}, S D=7.01\right)$ and Day $3-5$ (mean reach errors $\left.=22.9^{\circ}, S D=5.6\right)$ compared to Day $1\left(\right.$ mean reach errors $\left.=15.64^{\circ}, S D=6.11\right)($ Day 1 vs. Day 2, $p=0.0002$ and Day 1 vs. Day $3-5, p<0.0001$; see FIG. 8B). There was no difference in reaching performance on Day 2 vs. Day 3 - $5(p=0.57)$.

\subsubsection{Performance during Reach Training Trials}

PV reaching errors during the reach training trials are shown in FIG. 9. From the figure we see that participants quickly altered their movements when the distortion was presented, regardless of testing day. Performance was tracked through changes in peak velocity (PV) angular error and our savings analysis compared PV angular errors during initial reach training trials (Trials 2 - 3) across the first and last day of testing. Analysis revealed a main effect of Day $\left[F_{(1,22)}=6.022, p=0.023\right]$, such that participants had greater errors on Day 5 (mean PV angular errors $\left.=29.3^{\circ}, S D=7.27\right)$ compared to Day $1\left(\right.$ mean PV angular errors $\left.=24^{\circ}, S D=5.84\right)$, indicative of savings. There was no significant main effect of Group $\left[F_{(1,22)}=0.098, p=0.775\right]$, nor Day x Group interaction $\left[F_{(1,22)}=0.181, p=0.675\right]$.

\section{Discussion}

The goal of the present experiment was to examine retention of proprioceptive recalibration over an extended period of time (4 days) in the form of recall and savings. Additionally, we looked to determine the benefits of additional training on short-term retention of proprioceptive recalibration. Participants completed reach training trials (i.e. 150 reaches), during which they reached to a target when provided with rotated visual feedback of the hand (i.e., a cursor rotated $30^{\circ} \mathrm{CW}$ relative to the hand) on an initial day of testing. Following this initial day of testing, participants were divided into two groups: one which performed additional 


\section{LONG-TERM RETENTION OF PROPRIOCEPTIVE RECALIBRATION}

reach training trials across 4 consecutive days of testing (Training group) and another which did not perform the additional training (Non-Training group) on days 2 through 4; allowing us to assess recall of proprioceptive recalibration and examine the effects of additional training on recall across 4 consecutive days. On Day 5, all participants completed all reach training trials, allowing us to establish if proprioceptive recalibration was retained in the form of savings.

\subsection{Proprioceptive Recalibration and Retention}

Our data show an initial proprioceptive bias of about $6.2^{\circ}$ to the left of the visual reference marker following reach training with an aligned cursor, which is in line with previous literature (Cressman \& Henriques 2010; Jones et al. 2010; Zbib et al. 2016). More importantly, we found that following 150 reach training trials with a rotated cursor there was an overall change in proprioceptive biases of $12.5^{\circ}$ across all participants. These changes arose gradually, with performance changing across the first 40 reach training trials, after which we found no further significant differences between blocks (i.e. proprioceptive recalibration leveled off, or plateaued). The extent of proprioceptive recalibration in our experiment is only slightly higher (at $\sim 40 \%$ of distortion) than previously reported (e.g. $~ 30 \%$; Cressman \& Henriques 2009;

Salomonczyk et al. 2011, 2012; Zbib et al. 2016). Moreover, the time course of proprioceptive recalibration we observed on this first day of testing is consistent with findings by Zbib et al. (2016), showing small changes initially, that took a number of trials to plateau (specifically in Block 4 (40 trials) in the current experiment compared to Block 5 (70 trials) in the experiment by Zbib et al. 2016).

The proprioceptive recalibration observed on Day 1 was recalled to a small extent on Day 2 across all participants $\left(4.4^{\circ}\right.$ or $\sim 36.5 \%$ of the initial recalibration $)$. This recall is similar to previous results which have demonstrated the presence of proprioceptive changes related to 


\section{LONG-TERM RETENTION OF PROPRIOCEPTIVE RECALIBRATION}

shifts in felt hand position or motion up to 24 hours following reach training (Nourouzpour et al. 2015; Ostry et al. 2010). The current experiment extends these previous findings by establishing that proprioceptive recalibration was not retained 48 hours after initial reach training, and in fact dropped back down to baseline levels without additional training $\left(\sim 0^{\circ}\right)$. Despite the lack of recall of proprioceptive recalibration 48 hours after initial reach training, evidence of retention in the form of savings was present on Day 5 for the Non-Training group, as indicated by a faster rate of relearning and more traditional measures of savings (e.g. mean proprioceptive biases across the first 10 reach training trials were greater on Day 5 compared to Day 1). Together, these results suggest that retention in the form of savings, indicating faster relearning upon re-exposure to the perturbation, was not contingent on proprioceptive recalibration being recalled and was still present a relatively long time (4 days) after initial reach training.

In agreement with our experiment demonstrating long-term changes in the sensory system, Hatada et al. (2006) showed that one 75 minute session of reach training while looking through laterally displacing prisms lenses generated long-lasting sensory changes. Their sensory changes were determined by observing shifts in proprioceptively guided reaches, such that participants were required to actively point straight-ahead in the dark for 7 days following reach training. Immediately following reach training, reach errors to the proprioceptive target indicated changes of approximately $\sim 34 \%$ of the distortion. This shift in hand position was absent 6 hours after reach training. However, surprisingly, two days after completing the initial reach training (Day 3), shifts in sensed hand position were present once again and achieved their maximum, peaking at $38 \%$ of the distortion.

In contrast to Hatada and colleagues (2006), we did not find any increase in changes in proprioceptive biases across testing days. In fact, even though the extent of proprioceptive 


\section{LONG-TERM RETENTION OF PROPRIOCEPTIVE RECALIBRATION}

recalibration we found was much less than visuomotor adaptation on the first day of testing, it did not increase in magnitude over 4 additional days of testing. Specifically, for our Training group, recall and savings remained the same across Days 2 - 5 and the overall level of proprioceptive recalibration attained at the end of all testing days compared to baseline was nearly identical $(\sim 12)$. Thus, we found that regardless of performing additional training (specifically, 750 reach training trials across 5 consecutive testing days), proprioceptive recalibration never increased with respect to recall, savings or overall magnitude achieved. The differences observed between our results and those of Hatada et al. (2006) may be driven by the fact that their shifts in proprioception were assessed in a reaching task that might take into account adapted motor commands. Moreover, in the experiment by Hatada et al. (2006), visual feedback was distorted through the use of prisms, which cause a visual shift and hence realignment of the entire workspace, compared to the visual distortion introduced in the current experiment.

The ceiling we observe with respect to proprioceptive recalibration in a task that does not require a goal-directed movement has been observed previously in an experiment involving a single training session. Specifically, work by Salomoncyzk et al. (2011) found that proprioceptive recalibration did not increase across 3 blocks of 99 reach training trials. The current experiment extends these findings by demonstrating that proprioceptive recalibration does not benefit from additional training, even when completed on different testing days. Thus, the results speak to there being a point at which proprioceptive recalibration saturates, indicating a limit of changes in felt hand position, which is far less than changes in visuomotor adaptation. 


\section{LONG-TERM RETENTION OF PROPRIOCEPTIVE RECALIBRATION}

\subsection{Visuomotor Adaptation and Retention}

While the primary focus of the current research was to establish the potential of retention of proprioceptive recalibration, we also examined our reaching data to look for evidence of retention of visuomotor adaptation. We found that changes in reaches arose quickly. In fact, by examining our reach training data, we see that participants correctly aimed to the target within 5 training trials. This rapid visuomotor adaptation was reflected in the no-cursor reaches, as we found that after only 5 rotated reach training trials on the first day of testing, reach errors at Time 1 were significantly different compared to baseline (Non-Training group Time 1 Mean EP error $=21.8^{\circ}$; Training group Time 1 Mean EP error $=25.1^{\circ}$ ). Moreover, no further significant increases in visuomotor adaptation were observed over the course of training. These results are consistent with previous findings that have shown reach performance return to near baseline levels within just 20 training trials with a $30^{\circ}$ cursor rotation (Krakauer et al. 2000) and within 30 training trials with a $40^{\circ}$ cursor rotation (Yamamoto et al. 2006). Interestingly, similar to Zbib et al. (2016), we found that visuomotor adaptation decayed quickly, such that when sets of nocursor reaches were separated by 5 minutes, reach aftereffects at Time 2 were significantly less than those observed at Time 1 (Mean EP errors following the first block of rotated reach training: Non-Training group: Time $2=10.35^{\circ}$; Training group: Time $2=10.57^{\circ}$ ). This decay is also evident in the reach training data, as we saw that at the start of each reach training block (as seen in Fig 9), reach errors were far less than at the end of the previous block of reach training trials. This rapid decay of visuomotor adaptation was also observed in a control experiment conducted by Zbib et al. (2016), such that a similar decay in reach errors was seen for participants who were required to sit stationary, gripping the robot handle, instead of performing the proprioceptive estimation task. Thus, it seems that visuomotor adaptation decays over a 5 minute time interval, 


\section{LONG-TERM RETENTION OF PROPRIOCEPTIVE RECALIBRATION}

regardless of whether participants are completing a perceptual task or resting quietly. Moreover, we found that this decay continued even across additional training days.

In accordance with previous experiments (e.g. Nourouzpour et al. 2015), we found evidence of recall of visuomotor adaptation on Day 2 across participants. However, in contrast to the recall of proprioceptive recalibration, we found an increase in recall on Day 3 at Time 1 for our Training group and the Non-Training group maintained the extent of recall observed on Day 2. The Training group showed no further increase in recall across Days 4 and 5, and the NonTraining group maintained their level of recall across all testing days. In contrast to aftereffects observed at Time 1, aftereffects at Time 2 did not differ across days for either of the groups. This consistency at Time 2 is similar to results attained by Wong and Henriques (2009), who showed that extended training across 5 days did not lead to greater retention or adaptation. Specifically, like our findings, recall in the form of aftereffects observed on four consecutive days of testing following the initial training session did not vary and the magnitude of visuomotor adaptation observed at the end of the $5^{\text {th }}$ day of reach training did not differ from that observed at the end of the first, initial day of testing.

Reaching data in both the reach training trials and no-cursor reaching trials was examined for evidence of savings. As shown by Krakauer et al. (2005), we found evidence of savings in our reach training data, such that participants were quicker to correct their reaches in response to the rotated cursor on Day 5 compared to the initial day of testing. We did not examine our nocursor reaches at Time 1 for evidence of savings, as participants had adapted their reaches within 5 reach training trials and no further increases in errors were seen across the training sessions. However, reach errors were not as quick to plateau at Time 2, enabling us to examine the data for savings. While we did not find evidence of savings at Time 2 for the Non-Training group on Day 


\section{LONG-TERM RETENTION OF PROPRIOCEPTIVE RECALIBRATION}

5 vs. Day 1, reach errors were slightly quicker to plateau and were greater on subsequent testing days (e.g. Day 2 - 5 vs. Day 1) for our Training group. Taken together, our reach training and nocursor reaches provide evidence for both short (24 hours) and long (96 hours) retention of visuomotor adaptation in the form of recall and savings.

\subsection{Mechanisms underlying Visuomotor Adaptation and Proprioceptive Recalibration}

Here we have shown that proprioceptive recalibration and visuomotor adaptation arise with different time courses while training with altered visual feedback of the hand. The different time courses observed in the current experiment, and as seen previously by Zbib et al. (2016), would suggest that proprioceptive recalibration and visuomotor adaptation are driven by different error signals during visuomotor adaptation. Specifically, visuomotor adaptation has been suggested to arise due to error-based learning, which involves the reduction of the difference between the predicted and sensory consequences of a participant's movements (Tseng et al. 2007). In contrast, proprioceptive recalibration has been suggested to arise due to a crosssensory error signal, which is driven by differences between visual and proprioceptive estimates of limb position (Cressman \& Henriques 2010; Henriques \& Cressman 2012; Salomonczyk et al. 2013). A review article by Shadmehr and Krakauer (2008) provides some insight into where these error signals may be processed. Specifically, Shadmehr and Krakauer proposed that the cerebellum is involved in building an internal model that predicts the sensory consequences of a motor command and corrects these motor commands through sensory feedback, while the parietal cortex is involved in integrating the predicted visual and proprioceptive feedback with sensory feedback to estimate the current state of the body and the environment (i.e. resolving the cross-sensory error signal). Our results suggest that it takes longer for the brain to adapt to this 


\section{LONG-TERM RETENTION OF PROPRIOCEPTIVE RECALIBRATION}

cross-sensory conflict and the conflict is not resolved to the same extent as a visuomotor error signal, even with additional training.

We also observed different trends with respect to retention of proprioceptive recalibration compared to visuomotor adaptation. Specifically, while we found recall and savings of proprioceptive recalibration, these processes did not benefit from additional training. In contrast, recall of visuomotor adaptation did benefit from additional training, such that reach errors were greater on Days 3 - 5 for the Training group compared to Day 1. Interestingly, changes in reaching performance at Time 2 were more comparable to changes in proprioceptive recalibration than reaching performance at Time 1.

Traditionally, visuomotor adaptation and retention of visuomotor adaptation are thought to arise as a result of two processes: (1) an initial fast process, which is potentially explicit (or strategic) in nature and leads to quick decreases in error when the perturbation is present and (2) a slow process, which is associated with implicit learning and is proposed to lead to aftereffects (Redding \& Wallace 1996, 2002, 2003; Redding et al. 2005; Taylor \& Ivry 2011; Taylor et al. 2014). In more recent models, Smith and colleagues (Hadjiosif \& Smith 2013a, b; Miyamoto et al. 2014) do not credit learning to explicit and implicit processes directly, but refer to contributions from a faster, labile component and a slower, temporally stable component. The fast component is proposed to lead to a rapid reduction of errors to quickly improve performance, but retains information poorly, while the slower component responds slowly to errors throughout training and retains information well, and is suggested to be responsible for learning. Given our participants' lack of awareness (as explained in Section 2.4.7), performance in the reach training trials and no-cursor reaches likely reflects these fast and slow processes. as opposed to the explicit and implicit processes mentioned above. Specifically, the decreases in 


\section{LONG-TERM RETENTION OF PROPRIOCEPTIVE RECALIBRATION}

visuomotor adaptation observed when comparing Time 1 to Time 2 seen here (and shown by

Zbib et al. (2016)) may reflect a decay of the faster, labile component, which has been shown to decay after about 16.5 seconds (Hadjiosif \& Smith 2013a). In contrast, reach errors at Time 2 may reflect the more temporally stable component of learning and hence may be a more accurate representation of long-term motor changes which result from an internal visuomotor remapping. Moreover, as suggested by Smith et al. (2006), we propose that this slow process is responsible for implicit retention of visuomotor adaptation both in the form of recall and savings.

Proprioceptive recalibration has also been proposed to arise due to implicit processes. In support of proprioception arising implicitly, Hart and Henriques (2016) showed no difference in proprioceptive recalibration between participants that were given an explicit strategy to counteract a $30^{\circ}$ clockwise rotation and participants that were not given any strategy. Moreover, proprioceptive recalibration has been seen in the absence of any motor error and hence direct awareness of changes in the environment (Cressman \& Henriques, 2010). The relationship between implicit processes driving visuomotor adaptation and proprioceptive recalibration (and resulting retention) is currently unclear. In the experiment by Cressman and Henriques (2010), they showed that changes in visuomotor adaptation were similar in magnitude and correlated with proprioceptive recalibration. If we examine our rates of change (illustrated in FIG. 7), our results show some overlap of the $95 \%$ confidence intervals related to the rates of change of proprioceptive recalibration and visuomotor adaptation at Time 2, specifically on testing days following initial training on Day 1 (i.e. the rates of change of proprioceptive recalibration and visuomotor adaptation on Day 2 are similar). Moreover, recall and savings of proprioceptive recalibration and visuomotor adaptation at Time 2 do not change across testing days. Thus, 


\section{LONG-TERM RETENTION OF PROPRIOCEPTIVE RECALIBRATION}

perhaps, the slow, implicit, process described by Smith and colleagues (2006) is consolidated and, is responsible for retention of proprioceptive recalibration and visuomotor adaptation.

Interestingly, we found evidence of savings of proprioceptive recalibration in the absence

of recall, suggesting that there might be two different processes underlying retention that operate simultaneously, but depend on different networks. A recent experiment by Mawase et al. (2017), proposed that separate neuronal networks drive recall and savings of motor adaptation, specifically during locomotor adaptation to a split-belt treadmill. Researchers found evidence of both recall and savings with respect to locomotor adaptation. Interestingly, recall of locomotor adaptation was shown to be correlated with thalamic-cortical connectivity following the baseline session, while savings was shown to be correlated with cerebellar-thalamic connectivity following the baseline session. In accordance, with multiple motor circuits being involved in motor adaptation, an experiment by Vahdat et al (2011), found that changes in the cerebellar cortex, primary motor cortex, and dorsal premotor cortex were associated with reach adaptation in a force-field paradigm. They further showed that changes in sense of limb motion was associated with neural activity in the second somatosensory cortex, ventral premotor cortex, and supplementary motor cortex. Our behavioural data would suggest that, like motor adaptation, the neural circuitry involved in the recall and savings of proprioceptive recalibration may differ. Although speculative, we suggest that these two neural circuits may be linked to the parietal cortex, as opposed to the cerebellum.

\section{Conclusion}

In the current experiment, we found retention of proprioceptive recalibration in the form of recall (24 hours post initial training) and savings ( 96 hours post initial training). Additional training did not lead to any further benefits of retention of proprioceptive recalibration. Based on 


\section{LONG-TERM RETENTION OF PROPRIOCEPTIVE RECALIBRATION}

our results, we suggest that retention of proprioceptive recalibration is driven by implicit processes and that there may be 2 distinct neural networks underlying recall and savings of proprioceptive recalibration. Finally, similar implicit processes may also be linked to the longterm retention of visuomotor adaptation. 


\section{LONG-TERM RETENTION OF PROPRIOCEPTIVE RECALIBRATION}

\section{References}

Baraduc, P and Wolpert, D. M. (2002). Adaptation to a Visuomotor Shift Depends on the Starting Posture. Journal of Neurophysiology, 88(2), 973-981.

Berniker, M. and Kording, K. P. (2011). Estimating the relevance of world disturbances to explain savings, interference and long-term motor adaptation effects. PloS Computational Biology, 7(1), e1002210.

Buch, E.R., Young, S., and Contreras-Vidal, J.L. (2003). Visuomotor adaptation in normal aging. Learning and Memory, 10, 55-63.

Caithness, G., Osu, R., Bays, P., Chase, H., Klassen, J., Kawato, M., Wolpert, D. M. and Flanagan, J. R. (2004). Failure to consolidate the consolidation theory of learning for sensorimotor adaptation tasks. Journal of Neuroscience, 24(40), 8662-8671.

Cressman, E. K. and Henriques, D.Y. (2009). Sensory recalibration of hand position following visuomotor adaptation. Journal of Neurophysiology, 102(6), 3505-3518.

Cressman, E. K. and Henriques, D.Y. (2010). Reach adaptation and proprioceptive recalibration following exposure to misaligned sensory input. Journal of Neurophysiology, 103(1), 1888- 1895.

Cressman, E. K., Salomonczyk, D., and Henriques, D.Y. (2010). Visuomotor adaptation and proprioceptive recalibration in older adults. Experimental Brain Research, 205(4), 533544.

Hatada, Y., Miall, R. C. and Rossetti, Y. (2006) Two waves of a long-lasting aftereffect of prism adaptation measured over 7 days. Experimental Brain Research 169(3), 417-426.

Henriques, D. Y. and Cressman, E. K. (2012). Visuomotor adaptation and proprioceptive recalibration. Journal of Motor Behaviour, 44(6), 435-444. 


\section{LONG-TERM RETENTION OF PROPRIOCEPTIVE RECALIBRATION}

Jeannerod, M. (2003). Action Monitoring and Forward Control of Movements. In: Michael Arbib (Ed.), Brain Theory and Neural Networks. Second Edition. Cambridge, Mass.

Jenkins, W. M., Merzenich, M. M., Ochs, M. T., Allard, T. and Guíc-Robles, E. (1990). Functional reorganization of primary somatosensory cortex in adult owl monkeys after behaviorally controlled tactile stimulation. Journal of Neurophysiology, 63(1), 82-104.

Jones, S.A., Cressman, E.K., and Henriques, D.Y. (2010). Proprioceptive localization of the left and right hands. Experimental Brain Research, 204(3), 373-383.

Kesten H. (1958). Accelerated stochastic approximation. The Annals of Mathematical Statistics 29(1), 41-59.

Klassen, J., Tong, C. and Flanagan, J. R. (2005). Learning and recall of incremental kinematic and dynamic sensorimotor transformations. Experimental Brain Research, 164(2), 250259.

Krakauer, J.W., Ghilardi, M.F., and Ghez, C. (1999). Independent learning of internal models for kinematic and dynamic control of reaching. Nature Neuroscience, 2(11), 1026-1031.

Krakauer, J.W., Pine, Z.M., Ghilardi, M.F., and Ghez, C. (2000). Learning of visuomotor transformations for vectorial planning of reaching trajectories. Journal of Neuroscience, 20(23), 8916-8924.

Krakauer, J.W., Ghez, C. and Ghilardi, M.F. (2005). Adaptation to visuomotor transformations: consolidation, interference, and forgetting. Journal of Neuroscience, 25(2), 473-478.

Krakauer, J. W. (2009). Motor learning and consolidation: The case of visuomotor rotation. Advances in Experimental Medicine and Biology, 629(1), 4-5-421. 


\section{LONG-TERM RETENTION OF PROPRIOCEPTIVE RECALIBRATION}

Martin, T. A., Keating, J. G., Goodkin, H. P., Bastian, A. J., and Thach, W. T. (1996) Throwing while looking through prisms. I. Focal olivocerebellar lesions impair adaptation. Brain: a journal of neurology, 119, 1183-1198.

Mattar, A. A., Nasir, S. M., Darainy, M., and Ostry, D. J. (2011). Sensory change following motor learning. Progress in Brain Research, 191, 31-44.

Mattar, A. A. G., Darainy, M. and Ostry D. J. (2013). Motor learning and its sensory effects: time course of perceptual change in its presence with gradual introduction of load. Journal of Neurophysiology, 109(3), 782-791.

Mawase, F., Bar-Haim, S. and Shmuelof, L. (2017). Formation of Long-Term Locomotor Memories is Associated with Functional Connectivity Changes in the CerebellarThalamic-Cortical Network. The Journal of Neuroscience 37(2), 349 - 361.

McDougle, S.D., Ivry, R. B. and Taylor, J.A. (2016). Taking aim at the cognitive side of learning in sensorimotor adaptation tasks. Trends in Cognitive Sciences, 20(7), 535-544.

Oldfield, R.C. (1971). The assessment and analysis of handedness: The Edinburgh inventory. Neuropsychologia, 9, 97-113.

Ostry, D. J., Darainy, M., Mattar, A. A., Wong, J., and Gribble, P.L. (2010). Somatosensory plasticity and motor learning. Journal of Neuroscience, 30(15), 5384-5393.

Recanzone, G. H., Merzenich, M. M. and Jenkins, W. M. (1992b). Frequency discrimination training engaging a restricted skin surface results in an emergence of a cutaneous response zone in cortical area 3a. Journal of Neurophysiology, 67(5), 1057-1070.

Recanzone, G. H., Merzenich, M. M., Jenkins, W. M., Grajski, K.A. and Dinse, H. R. (1992a). Topographic reorganization of the hand representation in cortical area $3 \mathrm{~b}$ owl monkeys 


\section{LONG-TERM RETENTION OF PROPRIOCEPTIVE RECALIBRATION}

trained in a frequency-discrimination task. Journal of Neurophysiology, 67(5), 10311056.

Redding G.M., and Wallace, B. (1996). Adaptive spatial alignment and strategic perceptualmotor control. Journal of Experimental Psychology, 22(2), 379-394.

Redding G.M., and Wallace, B. (2002). Strategic calibration and spatial alignment: A model from prism adaptation. Journal of Motor Behaviour, 34(2), 126-38.

Redding G.M., and Wallace, B. (2003). Dual prism adaptation: calibration or alignment? Journal of Motor Behaviour, 35(4), 399-408.

Redding, G.M., Rossetti, Y. and Wallace, B. (2005). Applications of prism adaptation: a tutorial in theory and method. Neuroscience and Biobehavioural Reviews, 29(3), 431-444.

Ruttle, J. E., Cressman, E. K. Hart, B. M. and Henriques, Y. P. (2016). Time Course of Reach Adaptation and proprioceptive Recalibration during Visuomotor Learning. PLoS ONE, 11(10), e0163695. doi:10.1371/journal.pone.0163695.

Sainburg, R.L., and Wang, J. (2002). Interlimb transfer of visuomotor rotations: Independence of direction and final position information. Experimental Brain Research, 145(4), 437-447.

Salomonczyk D., Cressman E. K. and Henriques D. Y. (2011). Proprioceptive recalibration following prolonged training and increasing distortions in visuomotor adaptation. Neuropsychologia, 49(1), 3053-3062.

Salomonczyk D., Henriques D. Y., and Cressman E. K. (2012). Proprioceptive recalibration in the right and left hands following abrupt visuomotor adaptation. Experimental Brain Research 217(2), 187-196.

Shadmehr, R. and Krakauer J.W. (2008). A computation neuroanatomy for motor control. Experimental Brain Research 185(3), 359-381. 


\section{LONG-TERM RETENTION OF PROPRIOCEPTIVE RECALIBRATION}

Shadmehr, R. and Mussa-Ivaldi, F. (1994). Adaptive representation of dynamics during learning of a motor task. Journal of Neuroscience, 14(5), 3208-3224.

Simani, M. C., McGuire, L. M., and Sabes, P. N. (2007). Visual-shift adaptation is composed of separable sensory and task-dependent effects. Journal of Neurophysiology, 98(5), 28272841.

Smith, M.A., Ghazizadeh, A. and Shadmehr, R. (2006) Interacting Adaptive Processes with Different Timescales Underlie Short-Term Motor Learning. PLoS Computational Biology, 4(6), e179.

Taylor, J.A. and Ivry, R.B. (2011). Flexible cognitive strategies during motor learning. PLoS Computational Biology, 7(3), e10001096.

Taylor, J.A., Krakauer, J.W., and Ivry, R.B. (2014). Explicit and implicit contributions to learning in a sensorimotor adaptation task. Journal of Neuroscience, 34(8), 3023- 3032.

Treutwein, B. (1995) Adaptive psychophysical procedures. Vision Research, 35(1), 2503-2522.

Tseng, Y.W., Diedrichsen, J., Krakauer, J.W., Shadmehr, R., Bastian, A.J. (2007). Sensory prediction errors drive cerebellum-dependent adaptation of reaching. Journal of Neurophysiology, 98(1), 54-62.

Vahdat, S., Darainy, M., Milner, T.E., and Ostry, D.J. (2011). Functionally specific changes in resting-state sensorimotor networks after motor learning. Journal of Neuroscience, 31(47), 16907-16915.

Van Beers, R. J., Wolpert, D. M., and Haggard, P. (2002). When feeling is more important than seeing in sensorimotor adaptation. Current Biology, 12, 834-837

Wong, T. and Henriques D. Y. (2009). Visuomotor Adaptation Does Not Recalibrate Kinesthetic Sense of Felt Hand Path. Journal of Neurophysiology, 101(2), 614-623. 


\section{LONG-TERM RETENTION OF PROPRIOCEPTIVE RECALIBRATION}

Yamamoto, K., Hoffman, D.S. and Strick, P. L. (2006). Rapid and long-lasting plasticity of input- output mapping. Journal of Neurophysiology, 96(5), 2797-2801.

Zbib, B., Henriques, D. Y. and Cressman, E. K. (2016). Proprioceptive recalibration arises slowly compared to reach adaptation. Experimental Brain Research, Experimental Brain Research, 234(8), 2201-2213. 
Figures
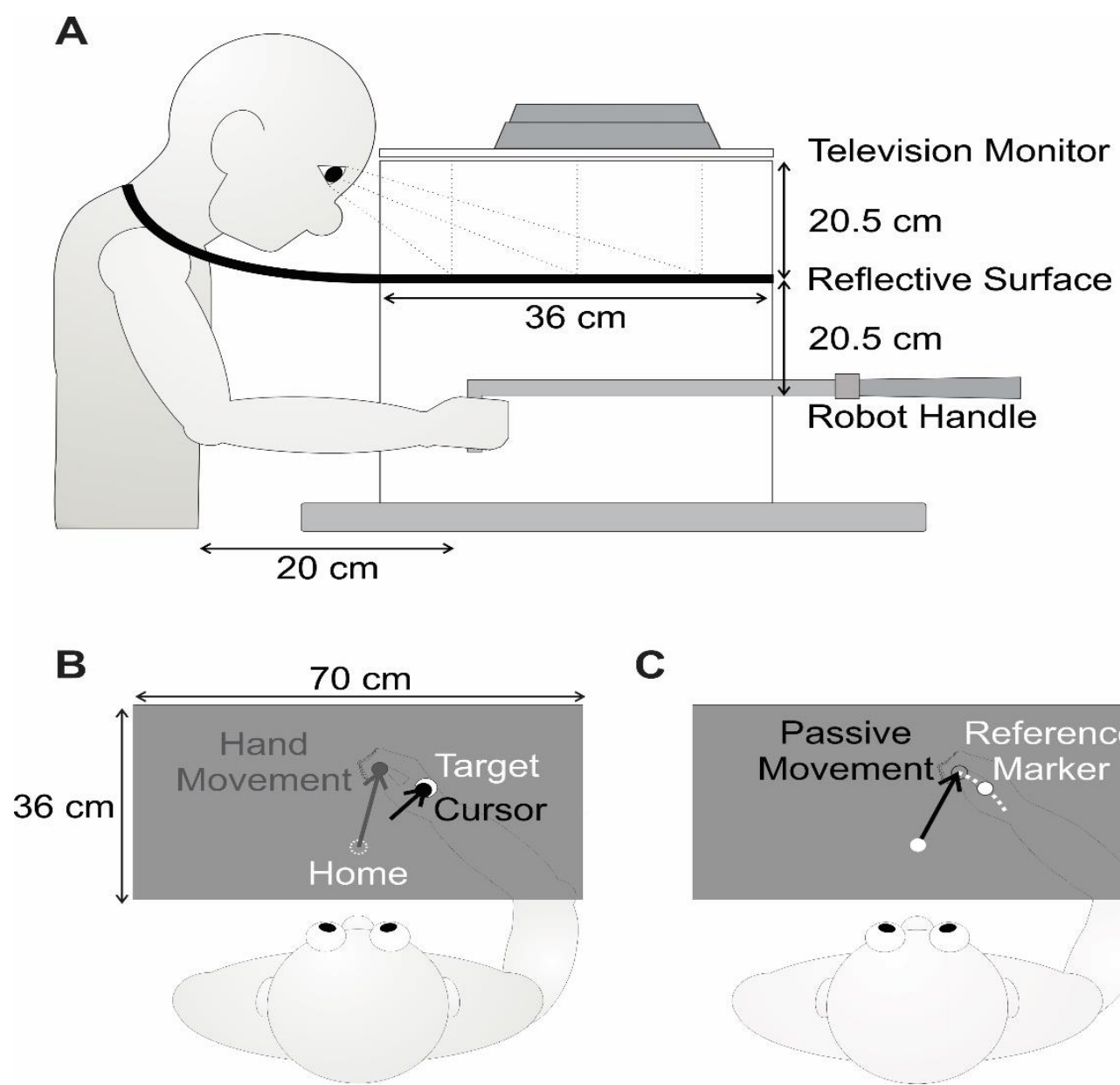

C

\section{FIG. 1. Experimental Set-Up}

Experimental set-up as seen from the side (A). Top view and the dimensions of the experimental surface visible to participants during reach training (B), and proprioceptive estimation (C) tasks. (B) During the reach training and no-cursor reach trials, the reach target (white circle; $2 \mathrm{~cm}$ diameter) was located $15 \mathrm{~cm}$ and $45^{\circ}$ clockwise $(\mathrm{CW})$ from the home position relative to straight ahead. During reach training trials, visual feedback appeared (green circle; $1 \mathrm{~cm}$ in diameter) once the hand had travelled $7 \mathrm{~cm}$ from the home position. For the rotated reach training trials, the cursor representing the participant's hand position was rotated $30^{\circ} \mathrm{CW}$ from the home position relative to straight ahead. (C) During the proprioceptive estimation trials the home position was visible at the beginning of the movement (white circle; $1 \mathrm{~cm}$ diameter). The reference marker (yellow circle; $1 \mathrm{~cm}$ diameter) was located $15 \mathrm{~cm}$ and $45^{\circ}$ clockwise from the home position relative to straight ahead. The hand was passively guided out by the robot to a position along the dotted white line using an adaptive staircase algorithm (note that the dotted line is displayed for illustration purposes only, it was not displayed to participants). The home position was removed once the hand began the guided movement and the reference marker was only displayed once the hand reached its final position. 


\section{LONG-TERM RETENTION OF PROPRIOCEPTIVE RECALIBRATION}

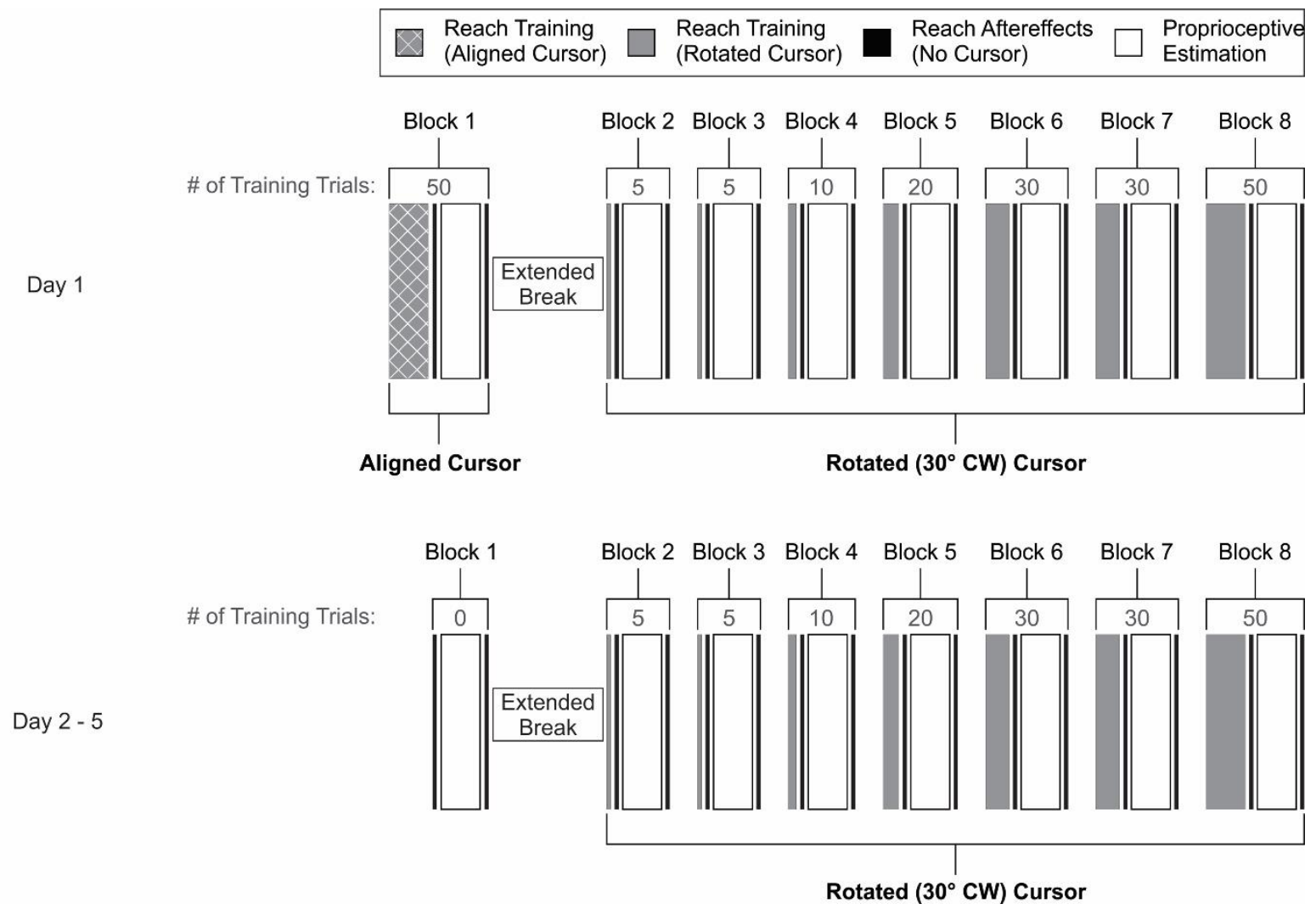

FIG. 2. Experimental Design

Breakdown of the testing blocks completed by the two groups (Non-Training and Training) within the experiment on Day 1 (top row) and Days $2-5$ (bottom row). Grey bars represent reach training trials when the cursor was aligned with the hand (diamond-crossed pattern) or rotated $30^{\circ}$ clockwise $(\mathrm{CW})$ relative to the hand (filled grey bars). The white bars represent sets of 50 proprioceptive estimate trials and black bars represent sets of 6 no-cursor reaching trials. The grey numbers indicate the number of reach training trials completed within each of the blocks. In each block, six no-cursor reaching trials occurred at Time 1, before proprioceptive estimate trials, and again at Time 2, after proprioceptive estimate trials. Days 2-5 did not contain aligned reach training trials as they were specific to Day 1 only. The Training group performed Blocks 1-8 on Days 2-5, while the Non-Training group performed only Block 1 on Days 2-4 and Blocks 1-8 on Day 5. 
A

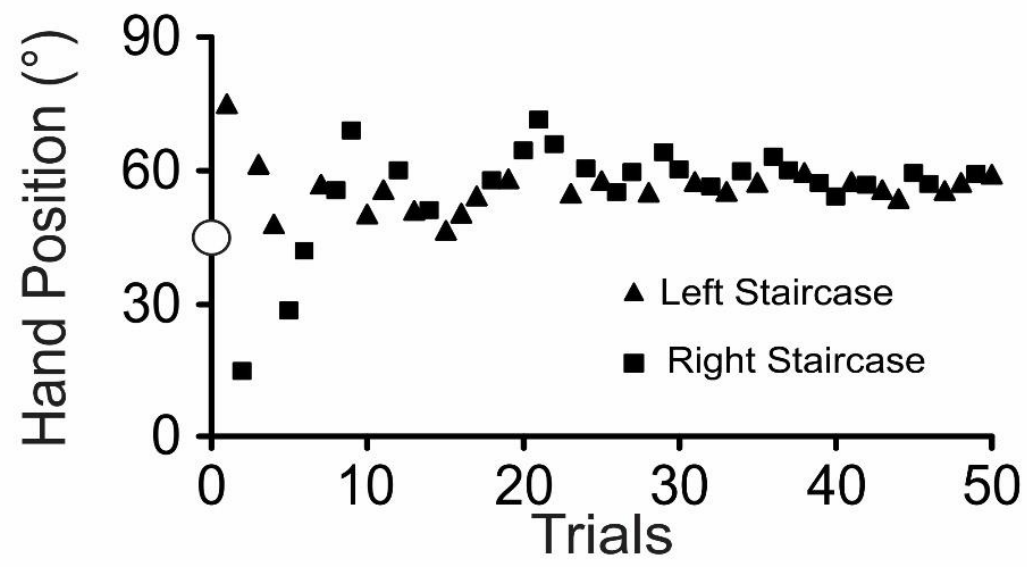

B

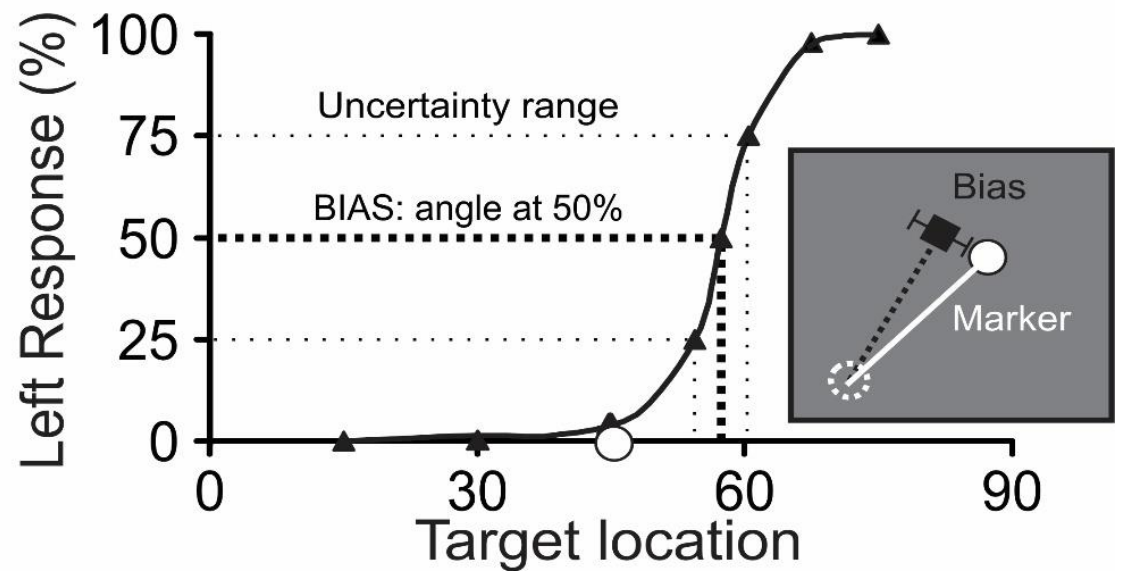

\section{FIG. 3. Proprioceptive Estimation Task}

An example of the positions a participant's hand was guided to during the proprioceptive estimation trials and their corresponding responses (A) The white circle on the y-axis represents the location of the reference marker located at $45^{\circ}$. An adaptive staircase algorithm was used to adjust hand positions according to whether the participant responded that their hand was left or right of the reference marker. One staircase began $20^{\circ}$ to the left of the target (triangles) and the other began $20^{\circ}$ to the right of the target (squares). (B) A logistic curve was fitted to a participant's responses to determine their bias (thick dashed black line) and their uncertainty range (difference between the thin dotted lines). In the inset to the right of the logistic function is an illustration showing the position of the reference marker, the participant's bias (black square) seen to be to the left of the reference marker and uncertainty range (thin lines). 

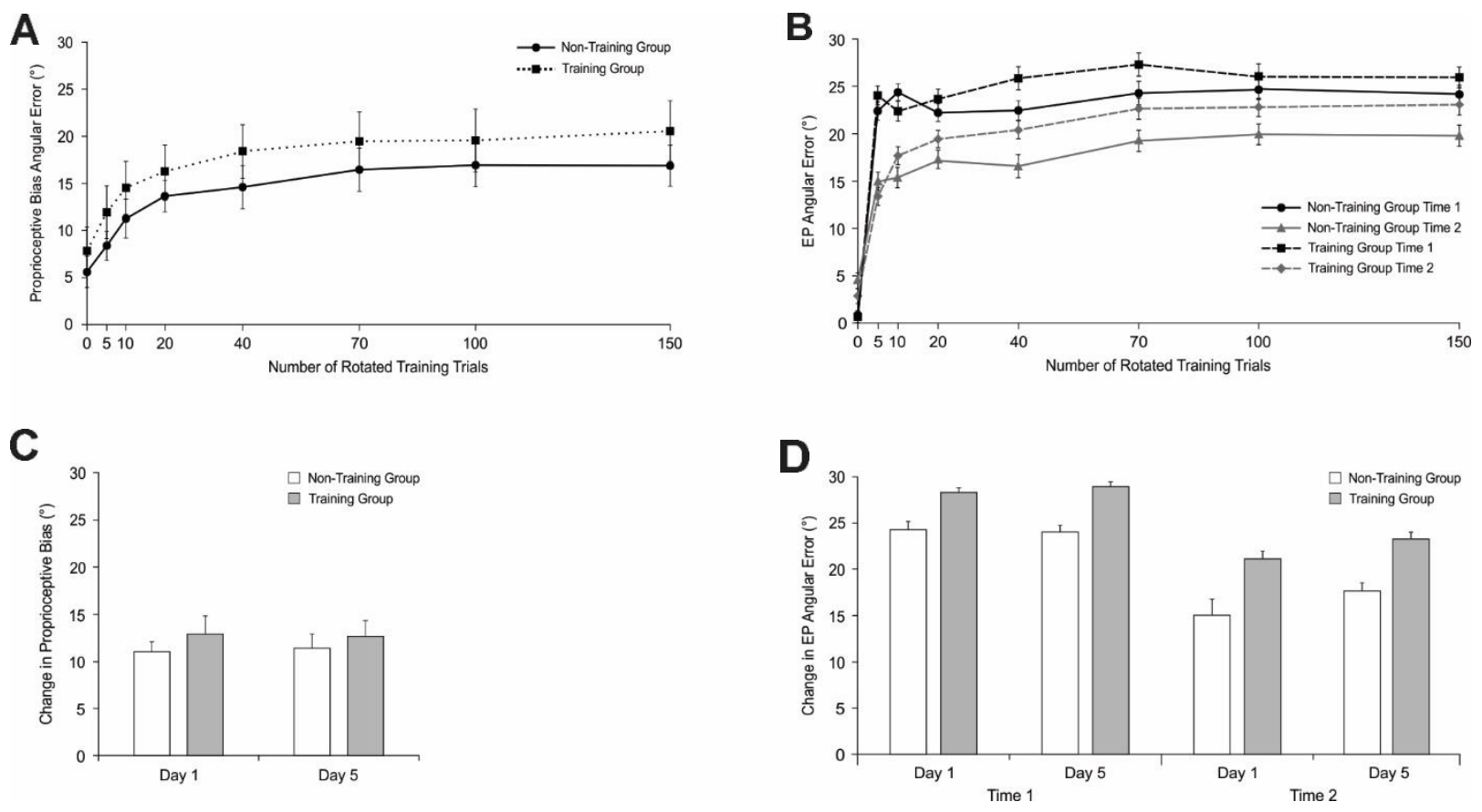

FIG. 4. Mean Proprioceptive Biases and Reach Endpoint Errors

(A) Mean proprioceptive biases (represented as angular errors in degrees relative to the reference marker) as a function of the number or reach training trials with the $30^{\circ} \mathrm{CW}$ rotated cursor for the Non-Training (NT) group (dotted line; squares) and the Training (T) group (solid line; circles) on Day 1. Data points corresponding to 0 rotated reach training trials represent the proprioceptive biases following aligned reach training trials (i.e. baseline). (B) Mean EP reach errors (represented as angular errors in degrees relative to the target) for the 6 no-cursor trials at Time 1 (NT group: black solid line; black circles, T group: black dashed line; black squares) and at Time 2 (NT group: grey solid line; grey triangles, T group: grey dashed line; grey diamonds) as a function of the number or reach training trials with the $30^{\circ} \mathrm{CW}$ rotated cursor. Again, data points corresponding to 0 rotated reach training trials reflect mean EP errors following aligned reach training trials (i.e. baseline). (C) Mean changes in proprioceptive biases achieved at the end of training (in Block 8) on Day 1 and Day 5 relative to proprioceptive biases in Block 1 on Day 1 (i.e. baseline) for the NT group (white) and T group (grey). (D) Mean change in EP angular errors during no-cursor trials at the end of training (in Block 8 at Time 1 and Time 2) on Day 1 and Day 5 relative to EP angular errors in Block 1 on Day 1 at Time 1 and Time 2 for the NT group (white) and T group (grey). Error bars reflect standard error of the mean. 


\section{LONG-TERM RETENTION OF PROPRIOCEPTIVE RECALIBRATION}
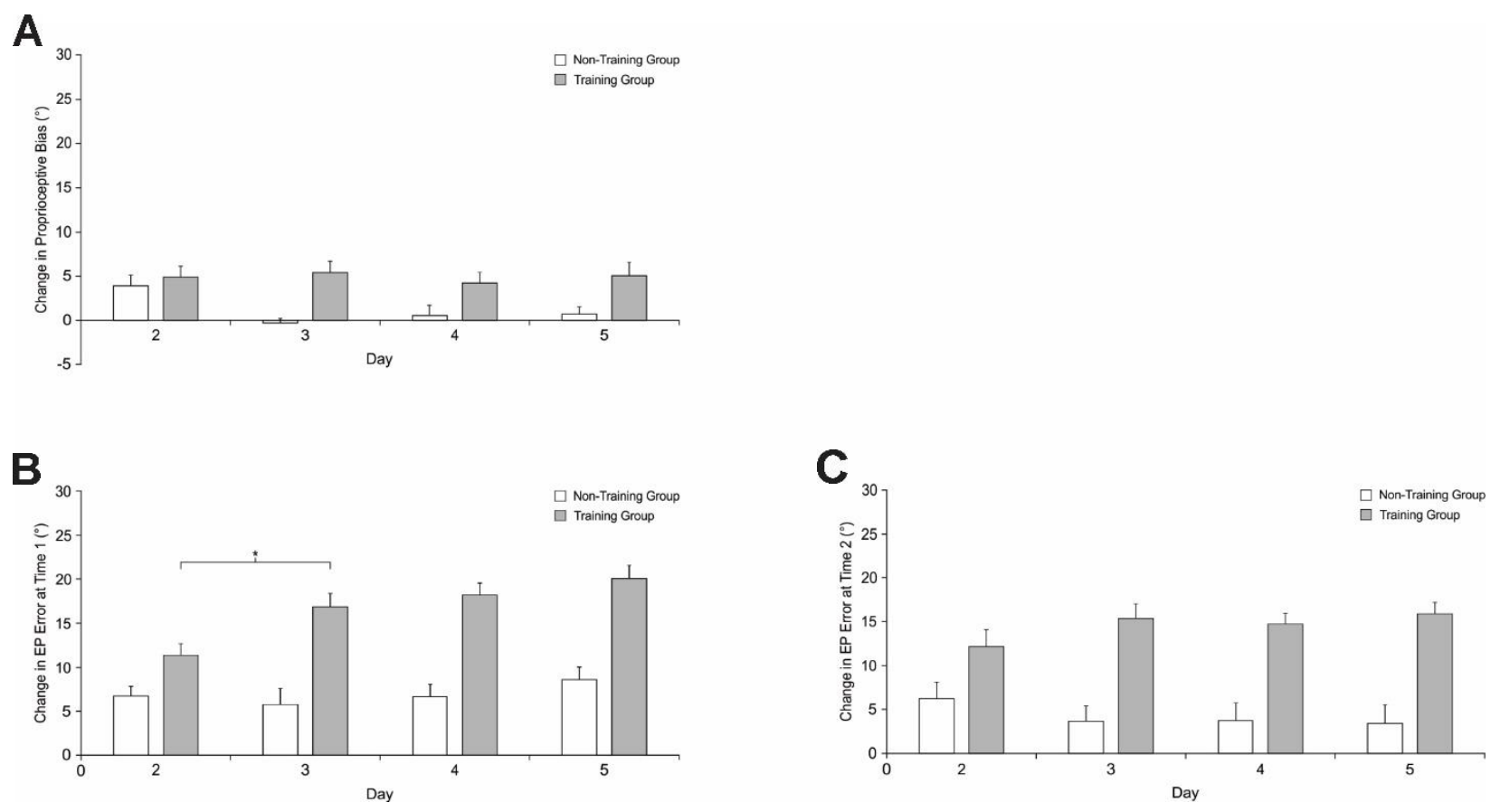

FIG. 5. Recall of Proprioceptive Recalibration and Visuomotor Adaptation

(A) Recall of mean proprioceptive biases across Days 2 through 5 for the Non-Training (NT) group (white) and the Training (T) group (grey) relative to proprioceptive biases in Block 1 on Day 1 (i.e. baseline). (B) Recall of mean EP errors for the no-cursor trials at Time 1 across Days 2 through 5 for the NT group (white) and the T group (grey) relative to mean EP errors in Block 1 on Day 1. (C) Recall of mean EP errors for the no-cursor trials at Time 2 across Days 2 through 5 for the NT group (white) and the T group (grey) relative to mean EP errors in Block 1 on Day 1. Error bars reflect standard error of the mean. Stars denote a significant difference ( $\mathrm{p}<$ $0.05)$. 

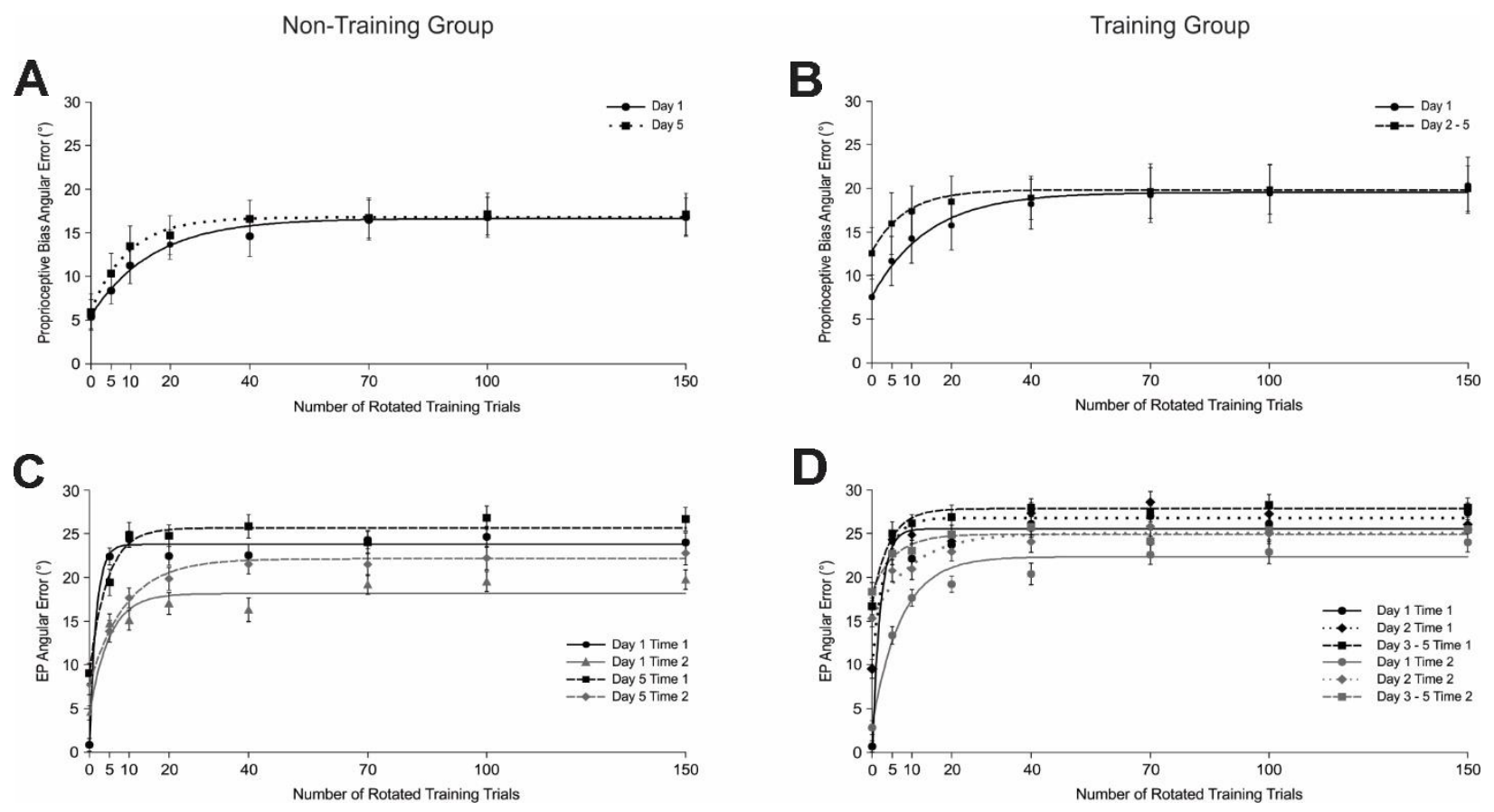

\section{FIG. 6. Mean Proprioceptive Biases and Reach Endpoint Errors across Testing Days}

(A) Mean proprioceptive biases (represented as angular errors in degrees relative to the reference marker) as a function of the number or reach training trials with the $30^{\circ} \mathrm{CW}$ rotated cursor for the Non-Training (NT) group on Day 1 (solid line; circles) and Day 5 (dotted line; squares). Data points corresponding to 0 rotated reach training trials represent the proprioceptive biases following aligned reach training trials on Day 1, or no rotated reach training on Day 5. (B) Mean proprioceptive biases as a function of the number or rotated reach training trials for the Training (T) group on Day 1 (solid line; circles) and mean performance on Days 2 - 5 (dashed line; squares). (C) Mean EP angular errors during the no-cursor trials as a function of the number of rotated reach training trials for the NT group on Day 1 (Time 1: black solid line; black circles \& Time 2: grey solid line; grey triangles) and Day 5 (Time 1: black dashed; black squares \& Time 2: grey dashed line; grey diamonds). (D) Mean EP angular errors during the no-cursor trials as a function of the number of rotated reach training trials for the $\mathrm{T}$ group on Day 1 (Time 1: black solid line; black circles \& Time 2: solid grey line; grey circles), Day 2 (Time 1: black dotted line; black diamonds \& Time 2: grey dotted line; grey diamonds) and Days 3 - 5 (Time 1: black dashed line; black squares \& Time 2: grey dotted line; grey diamonds). All curves represent exponential fits to the data per the equation used by Mattar et al. (2013). Error bars reflect standard error of the mean. 


\section{LONG-TERM RETENTION OF PROPRIOCEPTIVE RECALIBRATION}
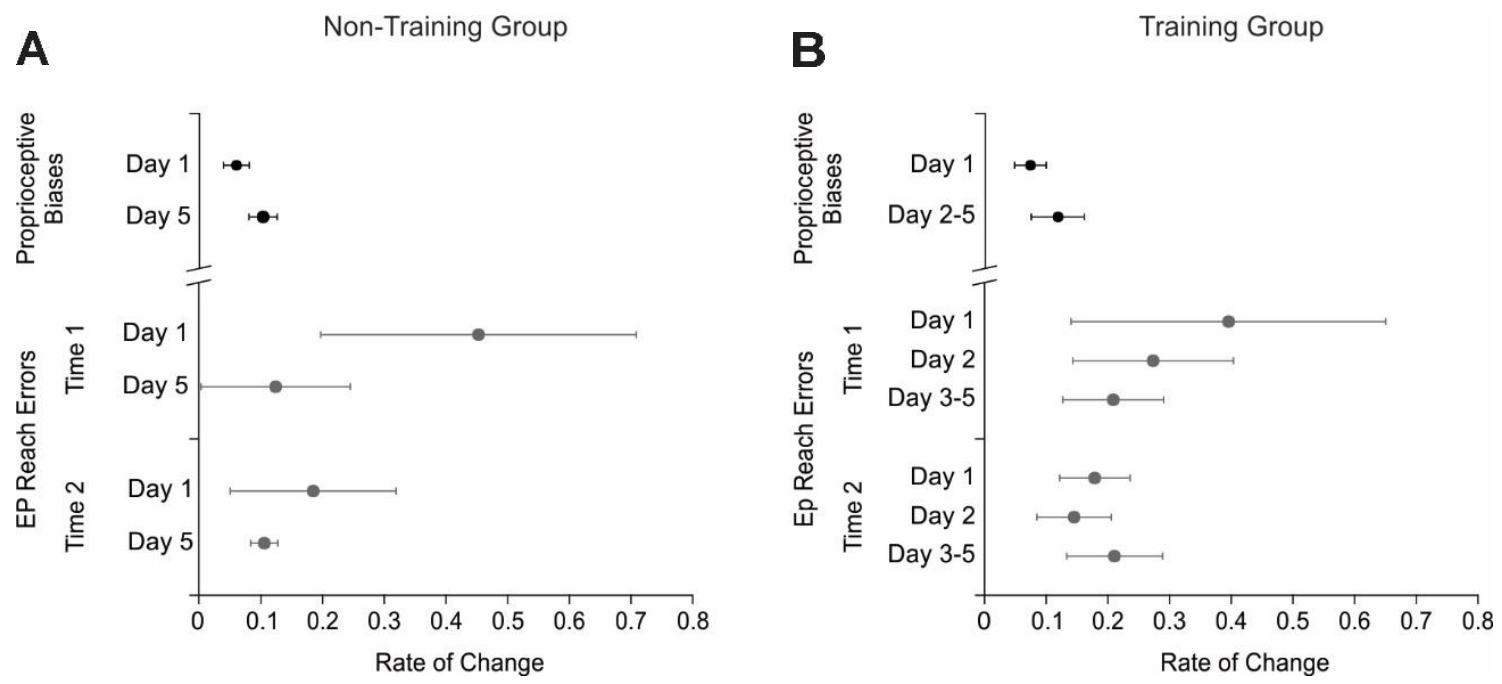

\section{FIG. 7. Rates of Change}

Rates of change for the exponential functions fit to the data shown in FIG. 6 for proprioceptive biases (black circles and lines) and EP errors (grey circles and lines) for (A) the Non-Training group and (B) Training group. The circles represent the rates of change and the lines indicate the $95 \%$ confidence intervals. 

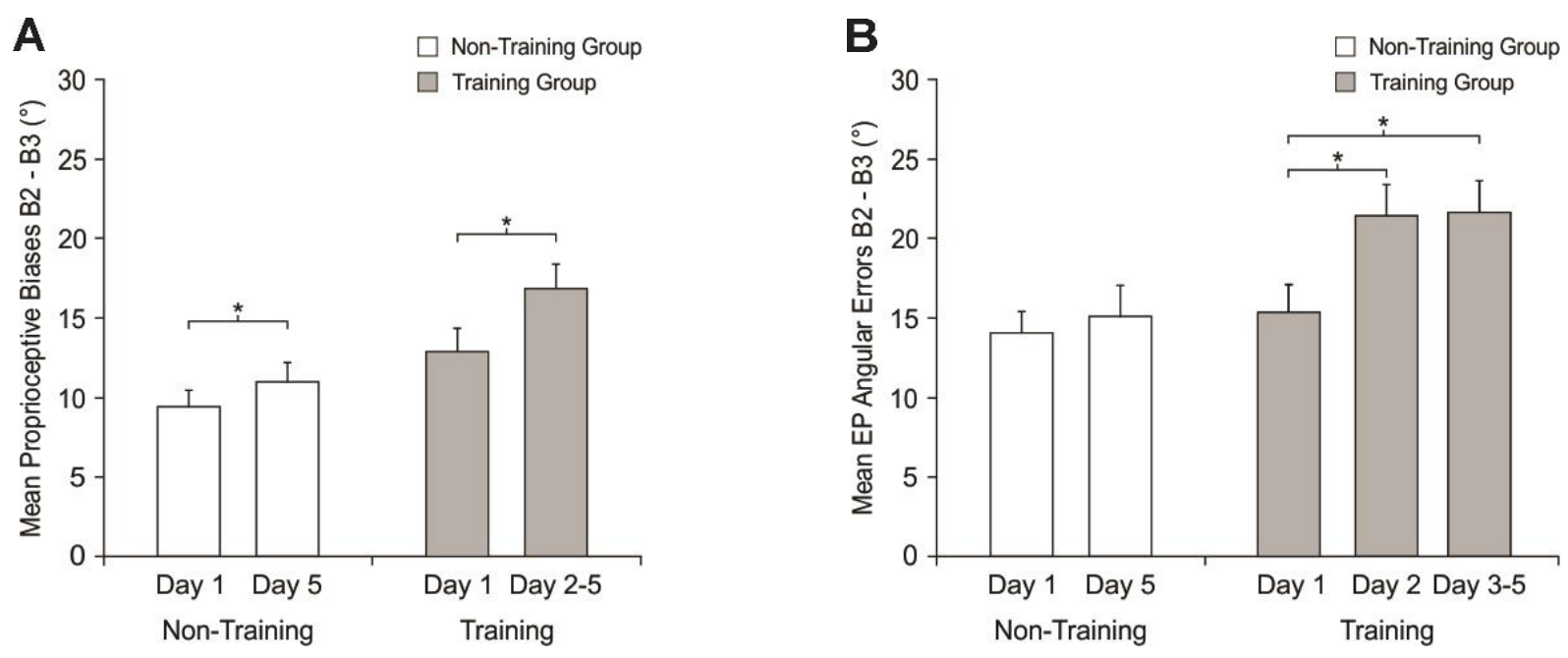

FIG. 8. Savings

Mean performance across Blocks 2 - 3 for participants in the Non-Training (white bars) and Training (grey bars) groups for (A) proprioceptive biases and (B) endpoint reach errors at Time 2. Error bars reflect standard error of the mean. Stars denote a significant difference $(p<0.05)$. 


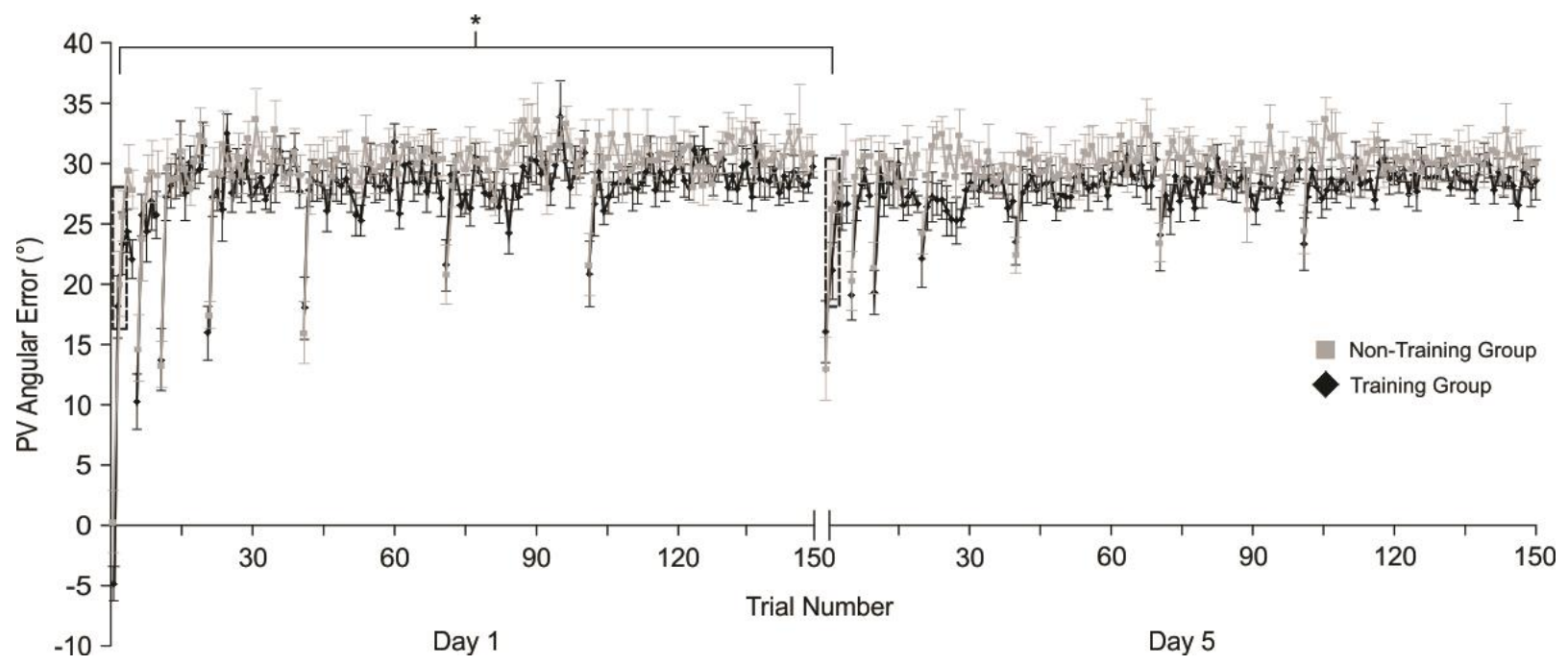

\section{FIG. 9. Reach Performance during Reach Training Trials}

Mean PV angular reach errors during reach training trials for the Non-Training group (grey squares) and the Training group (black diamonds) on Day 1 and Day 5 for all trials. Data are presented relative to mean PV angular reach errors achieved during reach training with an aligned cursor. Grey rectangles (with black dashed borders) highlight PV angular errors on initial trials (Trial 2 and 3), which were significantly different $(\mathrm{p}<0.05)$. Error bars reflect standard error of the mean. Note, the reduction of PV angular errors at the start of each block of reach training trials. 


\section{LONG-TERM RETENTION OF PROPRIOCEPTIVE RECALIBRATION}

\section{Chapter III: General Discussion}

Proprioception has been shown to be recalibrated following reaches to a visual target with misaligned visual feedback of the hand, such that one's sense of felt hand position is shifted in the direction of the visual feedback provided (Cressman \& Henriques 2009). The first objective of this experiment was to establish for how long proprioceptive recalibration is retained (over an extended period of time (i.e. 4 days)) after training to reach with distorted visual feedback of the hand. Then, we looked to determine whether additional training leads to benefits in proprioceptive recalibration in the form of recall and/or savings. Finally, we compared patterns of retention with respect to recall and savings, in order to determine if different underlying processes may underlie recall and savings.

As expected, many of our results are in agreement with previous experiments examining proprioceptive recalibration and visuomotor adaptation within a short period of time (e.g. 24 hours). Specifically, we found that participants recalibrated their sense of felt hand position in an initial day of testing by approximately $12^{\circ}$, which is similar to what has previously been shown (Cressman \& Henriques 2009; Salomonczyk et al. 2011, 2012; Zbib et al. 2016). Furthermore, the time course of proprioceptive recalibration observed on the first day of testing was consistent with findings by Zbib et al. (2016); such that proprioception was recalibrated much more slowly than visuomotor adaptation. In addition, we found recall of proprioceptive recalibration and visuomotor adaptation up to 24 hours following reach training, as seen by Nourouzpour et al. (2015). Finally, we found evidence of savings of visuomotor adaptation up to 4 days after initial training, as seen by Krakauer et al (2005). 


\section{LONG-TERM RETENTION OF PROPRIOCEPTIVE RECALIBRATION}

\section{Retention of Proprioceptive Recalibration vs. Retention of Visuomotor Adaptation}

The novelty of the current experiment lies in that we looked at retention of proprioceptive recalibration (and visuomotor adaptation) across an extended period of time following reach training with distorted visual feedback of the hand (i.e. 4 days). Furthermore, we examined and confirmed retention in the form of both recall and savings.

With regards to recall of proprioceptive recalibration, the Training group showed similar levels of recall across all testing days compared to the Non-Training group, which returned to baseline levels by the Day 3. As well, we found evidence of savings using multiple methods of analyses. Initially, we assessed savings by comparing rates of change of proprioceptive biases across training days. We also used more traditional methods for establishing savings (e.g. Krakauer et al. 2005), such that we compared when changes in proprioceptive biases plateaued (i.e. determined when proprioceptive biases did not differ from the final proprioceptive recalibration achieved), and compared participants' average performance over initial rotated reach training blocks without including data related to recall across training days (i.e. Blocks 2 3). All analyses clearly indicated that proprioceptive recalibration can be retained both in the form of recall and savings.

With regards to recall of visuomotor adaptation, the Training group had greater recall on Day 3 - 5 compared to Day 2 at Time 1, while recall seemed to decay by Time 2 . Interestingly, this observation of decay in recall between Time 1 and Time 2 (on Day 3-5) is similar to what is normally observed within a testing block during training. On the other hand, the Non-Training group maintained recall across all days at Time 1 and Time 2. We also found evidence of savings using multiple methods of analysis at Time 2. In the Training group, participants' reach errors levelled off earlier on Day 2 and then even earlier on Day 3 - 5. As well, reach adaptation 


\section{LONG-TERM RETENTION OF PROPRIOCEPTIVE RECALIBRATION}

measured during initial reach performance was greater on Day 3 - 5 compared to Day 1 and Day 2 (which was greater than Day 1). However, savings was not present in the Non-Training group. Analyses indicated that visuomotor adaptation at Time 2 can be retained both in the form of recall and savings in the Training group, while only recall was seen in the Non-Training group.

Based on our results, we suggest that visuomotor adaptation was influenced by two processes with differing learning rates; specifically, a fast and slow adaptive process as described by (Smith and colleagues 2006). The slow process (shown as the blue line in the left panel on FIG. 1) was responsible for the change in reaches towards the end of reach training, compared to the fast process (shown as the green line in the left panel on FIG. 1) which contributed initially to changes in reaches and then decayed quickly as training progressed. Furthermore, the fast and slow processes may have contributed to visuomotor adaptation assessed immediately following reach training at Time 1 (see FIG. 1), while performance following the five minute break (Time 2), may only have tapped into the slower process (as shown in FIG. 1). Similar to visuomotor adaptation at Time 2, we suggest that proprioceptive recalibration, which also arises gradually, is driven by the slow process (see FIG. 2). Then, following 24 hours, the slow process is responsible for retention of proprioceptive recalibration (right panel of FIG. 2) and visuomotor adaptation (right panel of FIG. 1) in both the form of recall and savings.

\section{Recall vs. Savings}

Upon analyzing performance in our Non-Training group, we observed that the timeline for the two forms of retention differed. Specifically, recall of proprioceptive recalibration was only evident 24 hours after initial training (not 48 hours), while savings was evident 96 hours after initial training. Evidence of savings in the absence of recall, suggests that these two forms of retention may be driven by distinct neural networks. Recent work has suggested that recall and 


\section{LONG-TERM RETENTION OF PROPRIOCEPTIVE RECALIBRATION}

savings of motor adaptation are likely mediated by two different neural networks (Mawase et al. 2017). Specifically, the experiment by Mawase et al. (2017), investigating the neuroanatomical correlates of recall and savings through resting-state fMRI scans, showed that baseline thalamiccortical connectivity was correlated with recall and that baseline cerebellar-thalamic connectivity was correlated with savings of locomotor adaptation (see right panel of FIG. 1). Based on our current results, we postulate that the sensory system also may have two separate neural networks associated with recall and savings (see FIG. 2). More specifically, these networks may be related to brain regions in the parietal lobe, such as the posterior parietal cortex or primary somatosensory cortex, as the region has been suggested to be responsible for integrating predicted visual and proprioceptive feedback with actual sensory feedback to estimate the current state of the body and its environment (Shadmehr \& Krakauer 2008). In other words, the parietal cortex would appear to be involved in the resolving the cross-sensory error signal proposed to drive proprioceptive recalibration (Henriques \& Cressman 2012).

Direct evidence for the parietal cortex having the potential to undergo long-term sensory plasticity comes from animal experiments involving sensory stimulation. Specifically, Recanzone et al. (1992a, b) investigated the responses of cortical neurons evoked by cutaneous stimulation in adult owl monkeys following training in a tactile frequency-discrimination task. They found that cortical representations of the trained hands in the primary somatosensory cortex (S1) were substantially more complex topographically than those of the unstimulated hand. Similar topographical reorganization had also been seen in monkeys who were taught to regulate their contact pressure and duration with a rotating disk to receive a food prize (Jenkins et al. 1990). These experiments demonstrate that long-term sensory changes in the parietal cortex are possible with extended training. 


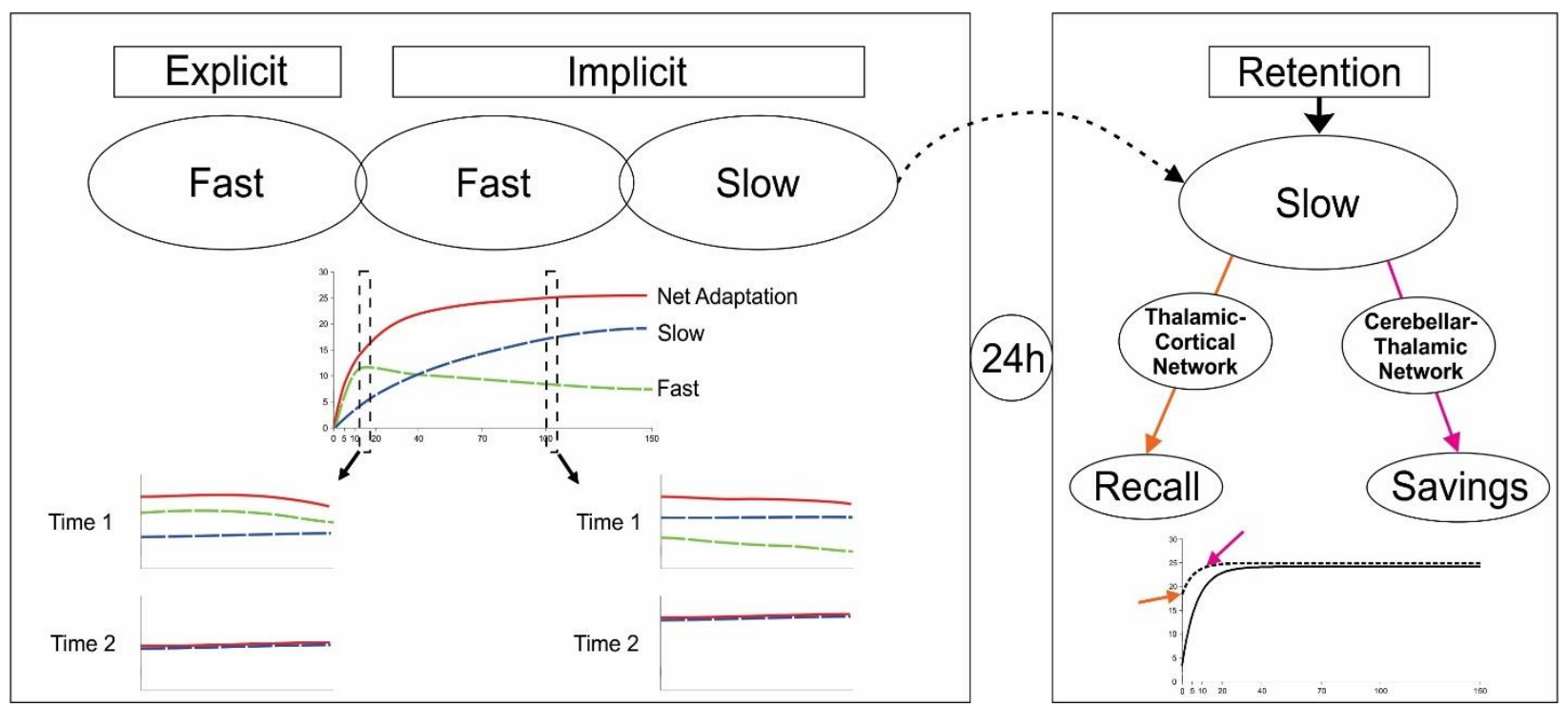

\section{FIG 1. Visuomotor Adaptation}

Illustrations of the proposed mechanisms underlying visuomotor adaptation and retention of visuomotor adaptation in the form of recall and savings according to Smith and colleagues 2006.

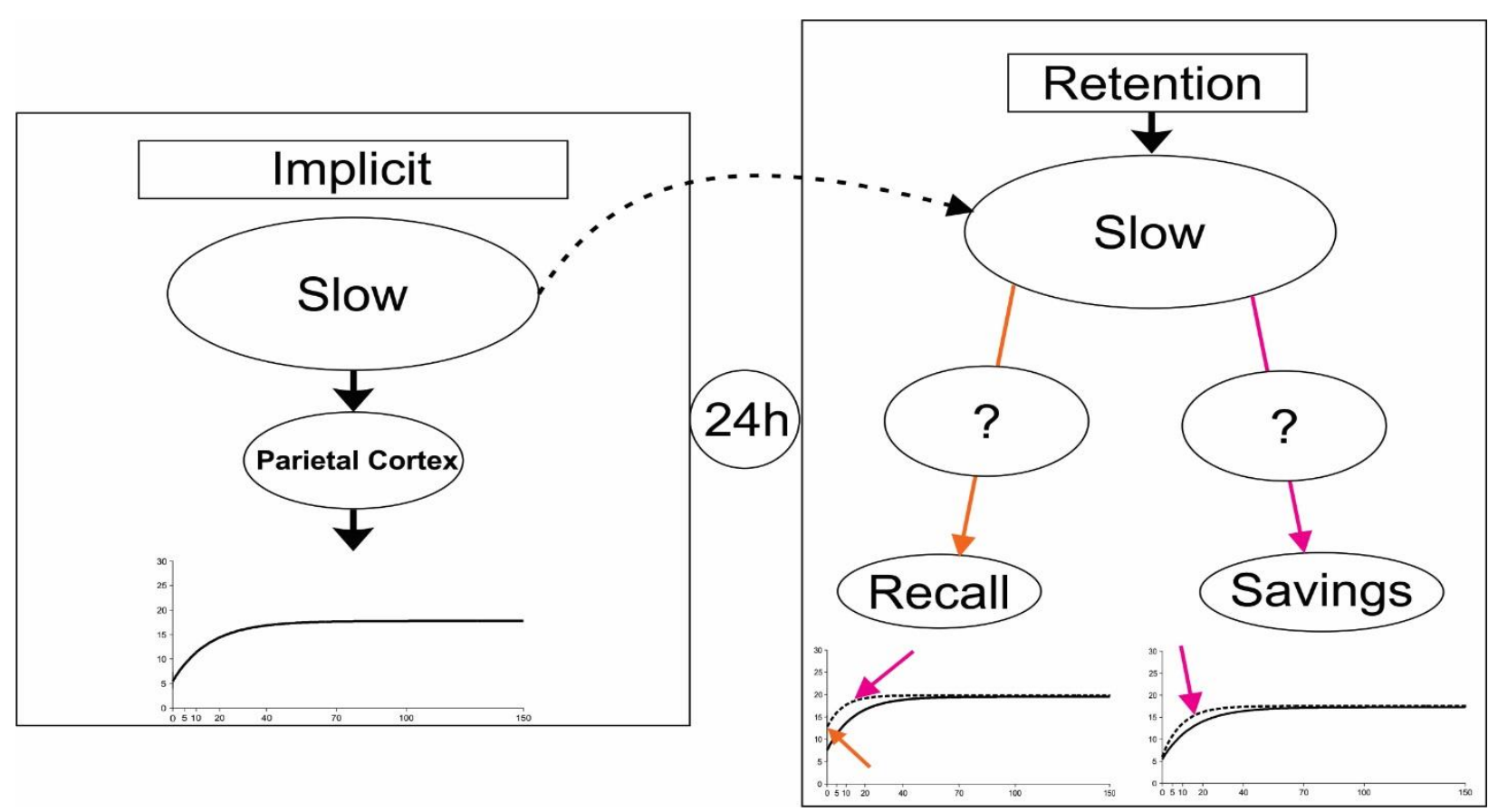

\section{FIG 2. Proprioceptive Recalibration}

Illustrations of our proposed mechanisms underlying proprioceptive recalibration and retention of proprioceptive recalibration in the form of recall and savings. 


\section{LONG-TERM RETENTION OF PROPRIOCEPTIVE RECALIBRATION}

\section{Limitations and Delimitations}

Factors we could not control across days were participant drop-out, the actions of participants outside of observation periods or potential decreases in participants' levels of motivation; which we were well aware could have occurred in our current experiment. Based on the consistency of participants' performance across days it does not seem that motivation levels dropped off during testing, however motivation was not monitored/assessed directly. A delimitation of the current experiment lies in how we determined proprioceptive biases. Biases were determined based on responses over 50 estimation trials, which took approximately 5 minutes to complete. Thus, there is a possibility for proprioceptive changes to occur over this time interval, as we saw with reach errors. A recent experiment by Ruttle et al. (2016), tried to counteract changes in proprioception influencing their results by using a proprioceptively guided reaching task (6 trials) to establish proprioceptive biases. However, using a reaching task is problematic because it involves actively reaching to measure proprioceptive recalibration, and hence potentially taps into adapted motor commands. While proprioceptive changes are a possibility in the current task, the proprioceptive estimation task we utilized avoids the issue of adapted motor commands influencing proprioceptive biases and has been used across a number of experiments (e.g. Cressman \& Henriques 2009, 2010; Cressman et al. 2010; Jones et al. 2010; Mattar et al 2011, 2013; Ostry et al. 2010; Salomonczyk et al. 2011).

In summary, the results from this experiment showed that additional training did not benefit retention of proprioceptive recalibration. However, we did find that retention of proprioceptive recalibration occurs in the form of both recall ( 24 hours after initial training) and savings (96 hours after initial training), suggesting that they are driven by distinct neural processes. These findings add to the body of literature regarding long-term retention of 


\section{LONG-TERM RETENTION OF PROPRIOCEPTIVE RECALIBRATION}

proprioceptive recalibration (and visuomotor adaptation). Further research is needed to determine the specific processes underlying retention of proprioceptive recalibration and its relationship to visuomotor adaptation.

\section{Future Directions}

Given that our participants did not report awareness of the perturbation, we would suggest that the proprioceptive recalibration we observed and the retention of it, were driven by implicit processes. However, we did not have participants perform a post-experiment questionnaire, as done by Benson et al. (2011), to confirm that they remained unaware of the distortion. Thus, there is the possibility that explicit processes/awareness may have contributed to proprioceptive recalibration. Moving forward, tracking awareness to further study implicit (and explicit) processes with respect to the retention of proprioceptive recalibration (and visuomotor adaptation) is something to consider.

Future research should also look at the outcome of a shortened training program. Surprisingly, we found that the sensory system's ability to change with additional training saturated early on, such that proprioceptive recalibration and retention of proprioceptive recalibration in the form of recall and savings did not increase with additional training. In contrast, visuomotor adaptation did show limited benefits of additional training and occurred much quicker. Changes in environments often require individuals to adapt their movements (e.g. adapting to an increased (or decreased) sensitivity of a computer mouse). Conversely, it is uncommon that individuals must update the perceived location of their limbs, and, thus, perhaps changes in the sensory system require more interaction with the novel environment and are less stable across days. For rehabilitation, this may be important. Post-stroke individuals are often able to do motor tasks, but their movements are impaired (e.g. inaccurate or misdirected), which 


\section{LONG-TERM RETENTION OF PROPRIOCEPTIVE RECALIBRATION}

could arise due to motor and/or sensory malfunction (Nelles, et al. 1999; Scalha et al. 2011). Thus, results from the current experiment would suggest that training regimens need to be individualized to target the system (i.e. motor or sensory) that requires the most attention. Moreover, if patients want to maintain sensory changes following a training session, our data would suggest that patients need to continually train the system. Though, patients may only require a few "refresher" trials on subsequent days to maintain the changes observed during rehabilitation. The benefits of a shortened training program would be of use from a practical perspective. 


\section{LONG-TERM RETENTION OF PROPRIOCEPTIVE RECALIBRATION}

\section{References}

Baraduc, P and Wolpert, D. M. (2002). Adaptation to a Visuomotor Shift Depends on the Starting Posture. Journal of Neurophysiology, 88(2), 973-981.

Benson, B. L., Anguera, J. A. and Seidler R. D. (2011). A spatial explicit strategy reduces error but interferes with sensorimotor adaptation. Journal of Neurophysiology, 105(6), 28432851.

Berniker, M. and Kording, K. P. (2011). Estimating the relevance of world disturbances to explain savings, interference and long-term motor adaptation effects. PloS Computational Biology, 7(1), e1002210.

Buch, E.R., Young, S. and Contreras-Vidal, J.L. (2003). Visuomotor adaptation in normal aging. Learning and Memory, 10, 55-63.

Caithness, G., Osu, R., Bays, P., Chase, H., Klassen, J., Kawato, M., Wolpert, D. M. and Flanagan, J. R. (2004). Failure to consolidate the consolidation theory of learning for sensorimotor adaptation tasks. Journal of Neuroscience, 24(40), 8662-8671.

Craske, B., and Gregg, S. J. (1966). Prism after-effects: Identical results for visual targets and unexposed limb. Nature, 212(5057), 104-105.

Cressman, E. K. and Henriques, D.Y. (2009). Sensory recalibration of hand position following visuomotor adaptation. Journal of Neurophysiology, 102(6), 3505-3518.

Cressman, E. K. and Henriques, D.Y. (2010). Reach adaptation and proprioceptive recalibration following exposure to misaligned sensory input. Journal of Neurophysiology, 103(1), 1888- 1895. 


\section{LONG-TERM RETENTION OF PROPRIOCEPTIVE RECALIBRATION}

Cressman, E. K., Salomonczyk, D. and Henriques, D.Y. (2010). Visuomotor adaptation and proprioceptive recalibration in older adults. Experimental Brain Research, 205(4), 533544.

Harris, C.S. (1963). Adaptation to displaced vision: Visual, motor, or proprioceptive change? Science, $140(1), 812-813$.

Harris, C.S. (1965). Perceptual adaptation to inverted, reversed, and displaced Vision. Psychological Review, 72(6), 419-444.

Hatada, Y., Miall, R. C. and Rossetti, Y. (2006) Two waves of a long-lasting aftereffect of prism adaptation measured over 7 days. Experimental Brain Research 169(3), 417-426.

Hay, J. C., and Pick, H. L. Jr. (1966). Visual and proprioceptive adaptation to optical displacement of the visual stimulus. Journal of Experimental Psychology, 71, 150-158

Henriques, D. Y. and Cressman, E. K. (2012). Visuomotor adaptation and proprioceptive recalibration. Journal of Motor Behaviour, 44(6), 435-444.

Jeannerod, M. (2003). Action Monitoring and Forward Control of Movements. In: Michael Arbib (Ed.), Brain Theory and Neural Networks. Second Edition. Cambridge, Mass.

Jenkins, W. M., Merzenich, M. M., Ochs, M. T., Allard, T. and Guíc-Robles, E. (1990). Functional reorganization of primary somatosensory cortex in adult owl monkeys after behaviorally controlled tactile stimulation. Journal of Neurophysiology, 63(1), 82104.

Jones, S.A., Cressman, E.K., and Henriques, D.Y. (2010). Proprioceptive localization of the left and right hands. Experimental Brain Research, 204(3), 373-383. 


\section{LONG-TERM RETENTION OF PROPRIOCEPTIVE RECALIBRATION}

Klassen, J., Tong, C. and Flanagan, J. R. (2005). Learning and recall of incremental kinematic and dynamic sensorimotor transformations. Experimental Brain Research, 164(2), 250259.

Krakauer, J.W., Ghilardi, M.F., and Ghez, C. (1999). Independent learning of internal models for kinematic and dynamic control of reaching. Nature Neuroscience, 2(11), 1026-1031.

Krakauer, J.W., Pine, Z.M., Ghilardi, M.F., and Ghez, C. (2000). Learning of visuomotor transformations for vectorial planning of reaching trajectories. Journal of Neuroscience, 20(23), 8916-8924.

Krakauer, J.W., Ghez, C. and Ghilardi, M.F. (2005). Adaptation to visuomotor transformations: consolidation, interference, and forgetting. Journal of Neuroscience, 25(2), 473-478.

Krakauer, J. W. (2009). Motor learning and consolidation: The case of visuomotor rotation. Advances in Experimental Medicine and Biology, 629(1), 4-5-421.

Kojima, Y., Iwamoto, Y. and Yoshida, K. (2004). Memory of learning facilitates saccadic adaptation in the monkey. Journal of Neuroscience. 24(1), 7531-7539.

Lee, J. and Schweighofer, N. (2009). Dual Adaptation Supports a Parallel Architecture of Motor Memory. The Journal of Neuroscience, 29(33), 10396-10404.

Malone, L. A., Vasudevan E. V. and Bastian, A. J. (2011) Motor adaptation training for faster relearning. Journal of Neuroscience 31(42), 15136-15143.

Martin, T. A., Keating, J. G., Goodkin, H. P., Bastian, A. J., and Thach, W. T. (1996) Throwing while looking through prisms. I. Focal olivocerebellar lesions impair adaptation. Brain: a journal of neurology, 119, 1183-1198.

Mattar, A. A., Nasir, S. M., Darainy, M., and Ostry, D. J. (2011). Sensory change following motor learning. Progress in Brain Research, 191, 31-44. 


\section{LONG-TERM RETENTION OF PROPRIOCEPTIVE RECALIBRATION}

Mattar, A. A. G., Darainy, M. and Ostry D. J. (2013). Motor learning and its sensory effects: time course of perceptual change in its presence with gradual introduction of load. Journal of Neurophysiology, 109(3), 782-791.

Mawase, F., Shmuelof, L., Bar-Haim, S. and Karniel, A. (2014). Savings in locomotor adaptation explained by changes in learning parameters following initial adaptation. Journal of Neurophysiology, 111(7), $1444-1454$.

Mawase, F., Bar-Haim, S., Joubran K., Rubin, L., Karniel, A. and Shmuelof, L. (2016). Increased adaptation rates and reduction in trial-by-trial variability in subjects with cerebral palsy following a multi-session locomotor adaptation training. Frontiers in Human Neuroscience 10, 203.

Mawase, F., Bar-Haim, S. and Shmuelof, L. (2017). Formation of Long-Term Locomotor Memories is Associated with Functional Connectivity Changes in the CerebellarThalamic-Cortical Network. The Journal of Neuroscience 37(2), 349 - 361.

McDougle, S.D., Ivry, R. B. and Taylor, J.A. (2016). Taking aim at the cognitive side of learning in sensorimotor adaptation tasks. Trends in Cognitive Sciences, 20(7), 535-544.

Nelles, G., Spiekermann, G. Jueptner, M., Leonhardt., Müller, S., Gerhard, H. and Diener, C. H. (1999). Reorganization of Sensory and Motor Systems in Hemiplegic Stroke Patients. Stroke, 30(8), 1510-1516.

Oldfield, R.C. (1971). The assessment and analysis of handedness: The Edinburgh inventory. Neuropsychologia, 9, 97-113.

Ostry, D. J., Darainy, M., Mattar, A. A., Wong, J., and Gribble, P.L. (2010). Somatosensory plasticity and motor learning. Journal of Neuroscience, 30(15), 5384-5393. 


\section{LONG-TERM RETENTION OF PROPRIOCEPTIVE RECALIBRATION}

Pisella, L., Michel, C., Grea, H., Tilikete, C., Vighetto, A., and Rossetti, Y. (2004). Preserved prism adaptation in bilateral optic ataxia: strategic versus adaptive reaction to prisms. Experimental Brain Research, 156(4), 399-408.

Recanzone, G. H., Merzenich, M. M. and Jenkins, W. M. (1992b). Frequency discrimination training engaging a restricted skin surface results in an emergence of a cutaneous response zone in cortical area 3a. Journal of Neurophysiology, 67(5), 1057-1070.

Recanzone, G. H., Merzenich, M. M., Jenkins, W. M., Grajski, K.A. and Dinse, H. R. (1992a). Topographic reorganization of the hand representation in cortical area $3 b$ owl monkeys trained in a frequency-discrimination task. Journal of Neurophysiology, 67(5), 10311056.

Redding, G. M., and Wallace, B. (1988). Adaptive mechanisms in perceptual-motor coordination: Components of prism adaptation. Journal of Motor Behavior, 20(3), 242254.

Redding G.M., and Wallace, B. (1993). Adaptive coordination and alignment of eye and hand. Journal of Motor Behaviour, 25(2), 75-88.

Redding G.M., and Wallace, B. (1996). Adaptive spatial alignment and strategic perceptualmotor control. Journal of Experimental Psychology, 22(2), 379-394.

Redding G.M., and Wallace, B. (1997a). Prism adaptation during target pointing from visible and nonvisible starting locations. Journal of Motor Behaviour, 29(2), 119-130.

Redding G.M., and Wallace, B. (1997b). Adaptive Spatial Alignment. Mahwah, NJ: Erlbaum.

Redding G.M., and Wallace, B. (2002). Strategic calibration and spatial alignment: A model from prism adaptation. Journal of Motor Behaviour, 34(2), 126-38. 


\section{LONG-TERM RETENTION OF PROPRIOCEPTIVE RECALIBRATION}

Redding G.M., and Wallace, B. (2003). Dual prism adaptation: calibration or alignment? Journal of Motor Behaviour, 35(4), 399-408.

Redding, G. M., and Wallace, B. (2006). Generalization of prism adaptation. Journal of Experimental Psychology, 32(4), 1006-1022.

Redding, G.M., Rossetti, Y. and Wallace, B. (2005). Applications of prism adaptation: a tutorial in theory and method. Neuroscience and Biobehavioural Reviews, 29(3), 431-444.

Ruttle, J. E., Cressman, E. K. Hart, B. M. and Henriques, Y. P. (2016). Time Course of Reach Adaptation and proprioceptive Recalibration during Visuomotor Learning. PLoS ONE, 11(10), e0163695. doi:10.1371/journal.pone.0163695.

Sainburg, R.L., and Wang, J. (2002). Interlimb transfer of visuomotor rotations: Independence of direction and final position information. Experimental Brain Research, 145(4), 437-447.

Salomonczyk D., Cressman E. K. and Henriques D. Y. (2011). Proprioceptive recalibration following prolonged training and increasing distortions in visuomotor adaptation. Neuropsychologia, 49(1), 3053-3062.

Salomonczyk D., Henriques D. Y., and Cressman E. K. (2012). Proprioceptive recalibration in the right and left hands following abrupt visuomotor adaptation. Experimental Brain Research 217(2), 187-196.

Scalha, B., Miyasaki, E., Lima, N., and Borges, G. (2011). Correlations between motor and sensory functions in upper limb chronic hemiparetics after stroke. Arquivos de Neuro Psiquiatria, 69(4), 624-629.

Shadmehr, R. and Krakauer J.W. (2008). A computation neuroanatomy for motor control. Experimental Brain Research 185(3), 359-381. 


\section{LONG-TERM RETENTION OF PROPRIOCEPTIVE RECALIBRATION}

Shadmehr, R. and Mussa-Ivaldi, F. (1994). Adaptive representation of dynamics during learning of a motor task. Journal of Neuroscience, 14(5), 3208-3224.

Simani, M. C., McGuire, L. M., and Sabes, P. N. (2007). Visual-shift adaptation is composed of separable sensory and task-dependent effects. Journal of Neurophysiology, 98(5), 28272841.

Smith, M.A., Ghazizadeh, A. and Shadmehr, R. (2006) Interacting Adaptive Processes with Different Timescales Underlie Short-Term Motor Learning. PLoS Computational Biology, 4(6), e179.

Taylor, J.A. and Ivry, R.B. (2011). Flexible cognitive strategies during motor learning. PLoS Computational Biology, 7(3), e10001096.

Taylor, J.A., Krakauer, J.W., and Ivry, R.B. (2014). Explicit and implicit contributions to learning in a sensorimotor adaptation task. Journal of Neuroscience, 34(8), 3023- 3032.

Van Beers, R. J., Wolpert, D. M., and Haggard, P. (2002). When feeling is more important than seeing in sensorimotor adaptation. Current Biology, 12, 834-837

Weiner, M.J., Hallet, M., and Funkenstein, H.H. (1983). Adaptation to lateral displacement of vision in patients with lesions of the central nervous system. Neurology, 33(6), 766-772.

Wolpert, D.M., Ghahramani, Z. and Jordan, M.I. (1995a). An internal model for sensorimotor integration. Science, 269(5232), 1880-1882.

Wolpert, D.M., Ghahramani, Z. and Jordan, M.I. (1995b). Are arm trajectories planned in kinematic or dynamic coordinates? An adaptation study. Experimental Brain Research, 103(3), 460-470.

Wolpert, D.M., Ghahramani, Z., and Flanagan, J.R. (2001). Perspectives and problems in motor learning. Trends in Cognitive Science, 5(11), 487-494. 


\section{LONG-TERM RETENTION OF PROPRIOCEPTIVE RECALIBRATION}

Wolpert, D.M. and Kawato, M. (1998). Multiple paired forward and inverse models for motor control. Neural Networks, 11(7-8), 1317-1329.

Wong, T. and Henriques D. Y. (2009). Visuomotor Adaptation Does Not Recalibrate Kinesthetic Sense of Felt Hand Path. Journal of Neurophysiology, 101(2), 614-623.

Yamamoto, K., Hoffman, D.S. and Strick, P. L. (2006). Rapid and long-lasting plasticity of input-output mapping. Journal of Neurophysiology, 96(5), 2797-2801.

Yin, P. B. and Kitazawa, S. (2001). Long-lasting aftereffects of prism adaptation in the monkey. Experimental Brain Research, 141(2), 25J0-253.

Zbib, B., Henriques, D. Y. and Cressman, E. K. (2016). Proprioceptive recalibration arises slowly compared to reach adaptation. Experimental Brain Research, Experimental Brain Research, 234(8), 2201-2213. 


\section{Appendix A}

\section{Edinburgh Handedness Inventory (Oldfield, 1971)}

Name:

Date:

Please indicate with a number (1 or 2 ) your preference in using your left or right hand in the following tasks.

Where the preference is so strong you would never use the other hand, unless absolutely forcedto, put the number 2 .

If you are indifferent, put a 1 in each column (1 | 1$)$.

Some of the activities require both hands. In these cases, the part of the task or object for which hand preference is wanted is indicated in parentheses.

\begin{tabular}{|l|l|l|}
\hline \multicolumn{1}{|c|}{ Task / Object } & \multicolumn{1}{c|}{ Left Hand } & \multicolumn{1}{c|}{ Right Hand } \\
\hline 1. Writing & & \\
\hline 2. Drawing & & \\
\hline 3. Throwing & & \\
\hline 4. Scissors & & \\
\hline 5. Toothbrush & & \\
\hline 6. Knife (without fork) & & \\
\hline 7. Spoon & RH $=$ \\
\hline 8. Broom (upper hand) & \\
\hline 9. Striking a Match (match) & \\
\hline 10. Opening a Box (lid) & Total checks: & $\mathrm{LH}=$ \\
\hline Cumulative Total & $\mathrm{CT}=\mathrm{LH}+\mathrm{RH}=$ \\
\hline Difference & $\mathrm{D}=\mathrm{RH}-\mathrm{LH}=$ \\
\hline $\begin{array}{r}\text { Interpretation: } \\
\text { Result }\end{array}$ & $\mathrm{R}=(\mathrm{D} / \mathrm{CT}) \times 100=$ \\
\hline $\begin{array}{r}\text { (Left Handed: } \mathrm{R}<-40) \\
\text { (Right Handed: } \mathrm{R}>+40)\end{array}$ & \\
\hline
\end{tabular}




\section{Appendix B}

File Number: H12-13-09

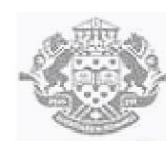

Date (mm/dd/yyyy): 01/11/2017

\section{Université d'Ottawa University of Ottawa \\ Bureau d'éthique et d'intégrité de la recherche \\ Office of Research Ethics and Integrity}

\section{Ethics Approval Notice}

\section{Health Sciences and Science REB}

Principal Investigator / Supervisor / Co-investigator(s) / Student(s)

$\begin{array}{llll}\text { First Name } & \text { Last Name } & \underline{\text { Affiliation }} & \underline{\text { Role }} \\ \text { Erin K. } & \text { Cressman } & \text { Health Sciences / Human Kinetics } & \text { Supervisor } \\ \text { Mohammad } & \text { Abu-Shaaban } & \text { Health Sciences / Human Kinetics } & \text { Student Researcher } \\ \text { Jean-Michel } & \text { Bouchard } & \text { Health Sciences / Human Kinetics } & \text { Student Researcher } \\ \text { Stefan } & \text { Maksimovic } & \text { Health Sciences / Human Kinetics } & \text { Student Researcher } \\ \text { Kristin-Marie } & \text { Neville } & \text { Health Sciences / Human Kinetics } & \text { Student Researcher }\end{array}$

File Number: H12-13-09

Type of Project: 4th year

Title: Motor Adaption and sensory recalibration

Renewal Date (mm/dd/yyyy)

$03 / 12 / 2016$

Special Conditions / Comments:

N/A
Expiry Date (mm/dd/yyyy)

$03 / 11 / 2017$
Approval Type

Renewal 


\section{LONG-TERM RETENTION OF PROPRIOCEPTIVE RECALIBRATION}

File Number: H12-13-09

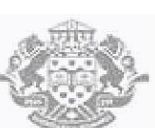

Date (mm/dd/yyyy): 01/11/2017

\section{Université d'Ottawa University of Ottawa \\ Bureau d'éthique et d'intégrité de la recherche \\ Office of Research Ethics and Integrity}

This is to confirm that the University of Ottawa Research Ethics Board identified above, which operates in accordance with the Tri-Council Policy Statement (2010) and other applicable laws and regulations in Ontario, has examined and approved the ethics application for the above named research project. Ethics approval is valid for the period indicated above and subject to the conditions listed in the section entitled "Special Conditions / Comments".

During the course of the project, the protocol may not be modified without prior written approval from the REB except when necessary to remove participants from immediate endangerment or when the modification(s) pertain to only administrative or logistical components of the project (e.g., change of telephone number). Investigators must also promptly alert the REB of any changes which increase the risk to participant(s), any changes which considerably affect the conduct of the project, all unanticipated and harmful events that occur, and new information that may negatively affect the conduct of the project and safety of the participant(s). Modifications to the project, including consent and recruitment documentation, should be submitted to the Ethics Office for approval using the "Modification to research project" form available at: http://research.uottawa.ca/ethics/submissions-and-reviews.

Please submit an annual report to the Ethics Office four weeks before the above-referenced expiry date to request a renewal of this ethics approval. To close the file, a final report must be submitted. These documents can be found at: http://research.uottawa.ca/ethics/submissions-and-reviews.

If you have any questions, please do not hesitate to contact the Ethics Office at extension 5387 or by e-mail at: ethics@uOttawa.ca.

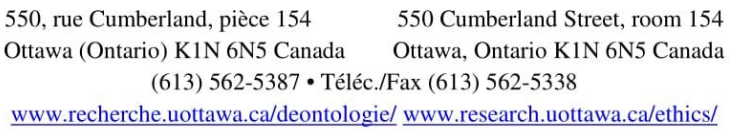




\title{
Appendix C
}

\section{Background information and consent form}

\section{Motor adaptation and sensory recalibration}

\author{
Supervisor: Dr. Erin Cressman, \\ Assistant Professor, \\ Montpetit Hall, Room 360 \\ School of Human Kinetics, University of Ottawa \\ Ottawa, Ontario, K1N 6N5
}

\section{Principal Investigator:}

Kristin-Marie Neville, M. Sc. Student, School of Human Kinetics, University of Ottawa

Stefan Maksimovic, M. Sc. Student, School of Human Kinetics, University of Ottawa

Mohammad Abu-Shaaban, 4th year Undergraduate Research Project Student, School of Human Kinetics, University of Ottawa

Jean-Michel Bouchard, 4th year Undergraduate Research Project Student, School of Human Kinetics, University of Ottawa

Funding: National Science and Engineering Research Council (NSERC)

\section{Background}

Most of us perform goal directed actions - i.e. reaching for a cup of coffee - without thought or effort. It is only after something goes wrong (i.e. damage to part of the brain), that we begin to appreciate how complex a job the brain has in transforming sensory signals (e.g. visual input) into appropriate action plans. The overall goal of the research being undertaken in my laboratory is to understand how the brain transforms sensory input into motor output in the "healthy" brain. This research has important implications for people suffering from neurological disorders, as it is only after we gain a fundamental understanding of the normal mechanisms underlying goal directed action that we can begin to design effective rehabilitation programs, targeted at individuals with damage to certain areas of the brain. 


\section{LONG-TERM RETENTION OF PROPRIOCEPTIVE RECALIBRATION}

\section{Purpose}

The purpose of this research is to determine how the brain combines multiple sources of sensory information so that it can plan an appropriate movement and form a coherent estimate of where the limb is in space. In particular, we are looking at the integration of visual and proprioceptive information in the brain, where proprioceptive input arises from sensory receptors inside the body that enable us to localize various parts of the body in relation to each other (e.g. proprioception is the sense that allows you to touch your index finger to your nose when your eyes are closed).

\section{Subject Profile}

To be a participant you must be a right-handed, healthy (no history of neurological, and/or motor dysfunction) adult with normal or corrected-to-normal visual function, aged between 16 and 40 years.

\section{Study Procedures}

In order to examine how visual and proprioceptive inputs are integrated in the brain you will be asked to point to targets (i.e. a visual dot) with your right/left hand and indicate where your hand is relative to a target (i.e. left or right). Your task will be to move and report your position as accurately as possible to the target. In order to record your hand movement, you will hold onto a robot handle (KINARM, Kingston, ON, Canada). We do not need to do any preparation of the skin. It is important to note that this robot system only captures the position of the handle (it does not capture your image (i.e. personal features) in any form).

\section{Experimental Session}

The entire experiment will be completed in Room 403 in Montpetit Hall. Each participant will complete the experiment either during a single testing session or during testing sessions on 5 consecutive days. Each testing session will range from 30 minutes up to a maximum of 2 hours. Thus, participants volunteering in the extended training groups will be devoting a maximum of 10 hours in total to this study across 5 consecutive days. Upon completion of the testing session(s) the experimenter will provide additional details on the visual information displayed and the hypothesized results. 


\section{LONG-TERM RETENTION OF PROPRIOCEPTIVE RECALIBRATION}

\section{Risks and Discomforts}

The risks involved in participating in this experiment are minimal. That is, the risks are no greater than the risks experienced in everyday life. However, you might experience slight fatigue, as you will be asked to maintain focused attention throughout the experiment and perform multiple reaching movements. In attempt to ensure that you do not become fatigued, we have scheduled 5 minute breaks approximately every 15 minutes. As well, please let us know if you require longer or more frequent periods of rest.

\section{Benefits}

Thank you for taking the time to participate in this study. While there are no direct benefits to you from participating in this study, this research has important implications for people suffering from neurological disorders. It is only after we gain a fundamental understanding of the normal mechanisms underlying sensory guided action that we can begin to design effective rehabilitation programs, targeted at individuals with damage to certain areas of the brain.

\section{Anonymity and Confidentiality}

All information and data collected are coded to maintain confidentiality. Specifically, raw data will be stored using an alphanumeric coding system so that no one will be able to identify you as your name will not appear on these files. The data will be analyzed on password protected computers that only the researchers directly involved in this study will have access to. Once analyzed the data will be kept in Room 403, Montpetit Hall, in locked filing cabinets and only the researchers directly involved in this study will have access to your data.

No records bearing your name will leave the institution. You are encouraged to request and discuss the results of the experimental trials at any time.

The data collected in this study may be published in scientific journals, as well as in a University of Ottawa MSc thesis. The data will be kept for a period of 10 years post-publication and will subsequently be destroyed by the physical resources service of the University of Ottawa.

\section{Voluntary Participation}

For the entire duration of the study, it is fully understood that you may refuse to participate or withdraw from the study at any time, without question. As well, you can ask the researcher any questions about any part of the research being conducted at any time. Data of participants who withdraw from the study will be destroyed immediately. 


\section{INFORMED CONSENT OF PARTICIPANT}

Research involving human subject require written consent of the participants.

$\mathrm{I}$, , hereby volunteer to participate as a subject in the study entitled "Motor adaptation and sensory recalibration". I have read the information presented in the above background information and I had the opportunity to ask questions to the investigators. I understand that my participation in this study, or indeed any research, may involve risks that are currently unforeseen.

I recognize that there will be no direct benefit to me from my participation in this study.

I understand that if I have any questions regarding the study, I may contact Dr. Erin Cressman. If I have any questions or complaints with regards to the ethical conduct of this study, I may contact the Protocol Officer for Ethics in Research, University of Ottawa, Tabaret Hall, 550 Cumberland Street, Room 154, Ottawa, ON K1N 6N5, tel.: 613-562-5387, email: ethics@uottawa.ca .

I have been given a copy of this Background Letter and Consent Form for me to keep.

Signature of participant:

Date:

Signature of Researcher:

Date: 


\section{LONG-TERM RETENTION OF PROPRIOCEPTIVE RECALIBRATION}

\section{Research Summary/Debriefing text}

The overall goals of this research are to examine the ability of the sensory and motor systems to learn and retain information over time.

The primary goal of this experiment was to investigate the ability of the sensory system to retain information over a long period of time (i.e. 5 days). To examine this, we had you reach to targets when provided with false visual feedback of the hand (e.g. reaching to a target while seeing a cursor that was rotated with respect to the hand's actual location). We then had you (1) reach to targets without any feedback to assess motor learning/adaptation, and (2) estimate where you felt your hand was located in space to determine sensory recalibration of the felt hand position. The study lasted 5 days in order to measure retention before and while the cursor perturbation was reintroduced (i.e. the maintenance of "aftereffects" and savings). These measurements allowed us to establish whether retention and savings occurred in the sensory system.

The second goal of the project was to examine if additional training benefited short-term sensory retention (i.e. 24 hours). Specifically, we are examining whether performing additional training on subsequent days compared to performing no training would benefit how well a person was able to retain information 24 hours later.

After having been briefed on the purpose of this research, do you consent to have your data included in this study?

Signature of participant:

Date:

Signature of Researcher:

Date: 